
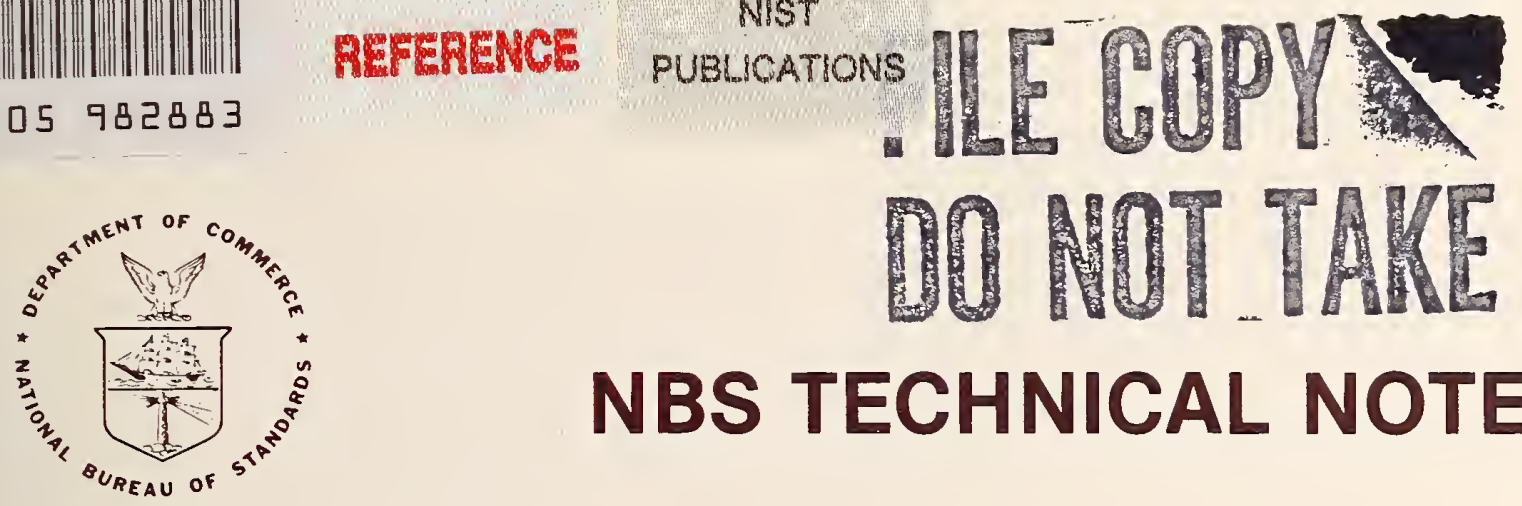

NBS TECHNICAL NOTE 1049

U.S. DEPARTMENT OF COMMERCE/National Bureau of Standards

\title{
A Study of Design Principles for Refrigerators for Low-Power Cryoelectronic Devices
}

CO

5753

. 1049

1982 


\section{NATIONAL BUREAU OF STANDARDS}

The National Bureau of Standards' was established by an act of Congress on March 3, 1901. The Bureau's overall goal is to strengthen and advance the Nation's science and technology and facilitate their effective application for public benefit. To this end, the Bureau conducts research and provides: (1) a basis for the Nation's physical measurement system, (2) scientitic and technological services for industry and government, (3) a technical basis for equity in trade, and (4) technical services to promote public safety. The Bureau's technical work is performed by the National Measurement Laboratory, the National Engineering Laboratory, and the Institute for Computer Sciences and Technology.

THE NATIONAL MEASUREMENT LABORATORY provides the national system of physical and chemical and materials measurement; coordinates the system with measurement systems of other nations and furnishes essential services leading to accurate and uniform physical and chemical measurement throughout the Nation's scientific community, industry, and commerce; conducts materials research leading to improved methods of measurement, standards, and data on the properties of materials needed by industry, commerce, educational institutions, and Government; provides advisory and research services to other Government agencies; develops, produces, and distributes Standard Reference Materials; and provides calibration services. The Laboratory consists of the following centers:

Absolute Physical Quantities² - Radiation Research - Thermodynamics and Molecular Science - Analytical Chemistry - Materials Science.

THE NATIONAL ENGINEERING LABORATORY provides technology and technicat services to the public and private sectors to address national needs and to sofve nationat problems; conducts research in engineering and applied science in support of these efforts; builds and maintains competence in the necessary disciplines required to carry out this research and technical service; develops engineering data and measurement capabifities; provides engineering measurement traceability services; develops test methods and proposes engineering standards and code changes; develops and proposes new engineering practices; and develops and improves mechanisms to transfer results of its research to the ultimate user. The Laboratory consists of the following centers:

Applied Mathematics - Electronics and Electrical Engineering ${ }^{2}$ - Mechanical Engineering and Process Technology ${ }^{2}$ - Building Technology - Fire Research Consumer Product Technology — Field Methods.

THE INSTITUTE FOR COMPUTER SCIENCES AND TECHNOLOGY conducts research and provides scientific and technical services to aid Federal agencies in the setection, acquisition, application, and use of computer technology to improve effectiveness and economy in Government operations in accordance with Public Law 89-306 (40 U.S.C. 759), relevant Executive Orders, and other directives; carries out this mission by managing the Federal Information Processing Standards Program, developing Federal ADP standards guidelines, and managing Federal participation in ADP voluntary standardization activities; provides scientific and technological advisory services and assistance to Federal agencies; and provides the technical foundation for computer-related policies of the Federal Government. The Institute consists of the following centers:

Programming Science and Technology - Computer Systems Engineering.

Headquarters and Laboratories at Gaithersburg, MD, unless otherwise noted; mailing address Washington, DC 20234.

${ }^{2}$ Some divisions within the center are located at Boulder, CO 80303. 


\section{A Study of Design Principles for Refrigerators for Low-Power Cryoelectronic Devices}

J.E. Zimmerman

D.B. Sullivan

Electromagnetic Technology Division National Engineering Laboratory National Bureau of Standards

Boulder, Colorado 80303

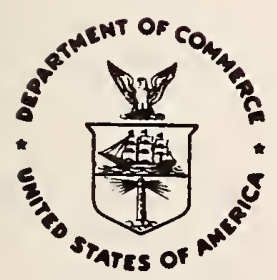

U.S. DEPARTMENT OF COMMERCE, Malcolm Baldrige, Secretary

NATIONAL BUREAU OF STANDARDS, Ernest Ambler, Director

Issued January 1982 
National Bureau of Standards Technical Note 1049

Nat. Bur. Stand. (U.S.), Tech. Note 1049, 114 pages (January 1982) CODEN:NBTNAE 


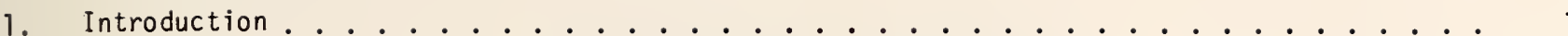

2. Background

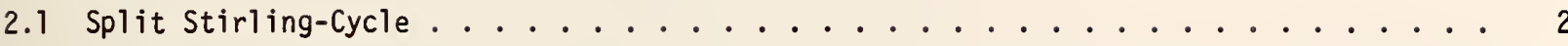

2.2 Twin-Piston Stirling Cycle . . . . . . . . . . . . . . . . . 4

3. Summary of Refrigerator Development

3.1 Split Stirling Cycle . . . . . . . . . . . . . . . . . . . 4 4

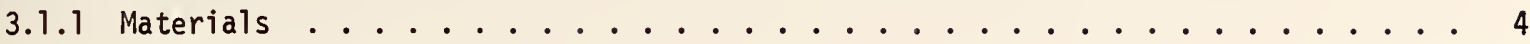

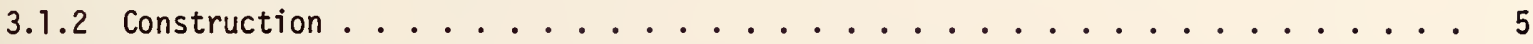

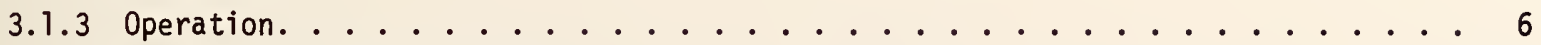

3.2 Twin-Piston Stirling Refrigerator. . . . . . . . . . . . . . 8

3.3 Compressor Design. . . . . . . . . . . . . . . . . . . 8

3.3.1 'O' Ring Sealed Piston with Conventional Crosshead ........... 8

3.3.2 Force Balanced Compressor. . . . . . . . . . . . . . . 9

3.3.3 Contamination Free Compressor. . . . . . . . . . . . . . 9

3.4 Temperature Control. . . . . . . . . . . . . . . . . . 10

4. Cryocooler Optimization

4.1 General Approach . . . . . . . . . . . . . . . . . . . . 11

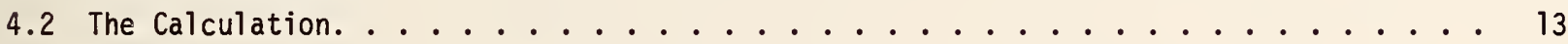

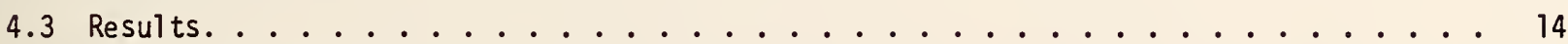

5. Summary and Conclusions. . . . . . . . . . . . . . . . . 17

6. References........................... . 17

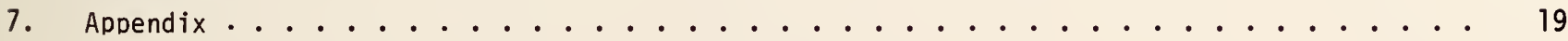





\title{
A Study of Design Principles for Refrigerators
}

For Low-Power Cryoelectronic Devices

\author{
J. E. Zimmerman and D. B. Sullivan \\ National Bureau of Standards \\ Electromagnetic Technology Division \\ Boulder, Colorado 80303
}

\begin{abstract}
This report summarizes a five-year effort at NBS which has been directed toward the development of low-power cryocoolers suited to the support of superconducting instruments. The report deals with a variety of aspects of construction and operation of refrigerators as well as with a model which allows one to optimize the design for minimum drive power. The publications generated by the program are included as an appendix.
\end{abstract}

Key words: cryocooler; cryogenics; low temperature; refrigerator; Stirling cycle; superconducting devices.

\section{Introduction}

This report describes a five-year effort at NBS to develop small, low-power Stirling crycooolers which are suited to the support of highly sensitive superconducting instrumentation, particularly magnetometers and magnetic gradiometers based on the Superconducting QUantum Interference Device (SQUID). The latter part of this first section describes the environmental requirements for such instrumentation. In Section 2 we provide a brief discussion of the operation of the cryocoolers and in Section 3 summarize the refrigerator development work. Section 4 describes a model which has been developed to optimize cryocooler design and finally the publications generated by the program are appended for completeness.

The new class of cryocoolers considered in this report were first described at two conferences in the summer of $1976[1,2]$. The machine described in these papers achieved a temperature of $13 \mathrm{~K}$ with three stages. The addition of a fourth stage reduced the bottom-end temperature to less than $8.5 \mathrm{~K}$ and a niobium SQUID magnetometer was operated with the cooler [3]. Analysis of the machine's operation was reported subsequently $[4,5]$. The operation of the cooler is limited by the internal thermal resistance of the plastic regenerator components, a situation much different than that for all other Stirling machines. In 1979, a low-pressure, twin-piston single stage cooler was built. This cooler succeeded in cooling from temperatures in the 8 to $12 \mathrm{~K}$ region to a bottom end temperature of 3 to $4 \mathrm{~K}$ [6]. This stage could conceivably be added to one of the higher-pressure ( 2 to 4 bars), multistage machines to achieve helium temperature operation. Finally we refer to a sequence of papers $[7,8,9$, 10$,$] which review and update the progress on this refrigeration work. The last of these papers$ describes a 5-stage refrigerator which achieves a bottom end temperature of less than $7 \mathrm{~K}$ with only 20 $W$ of mechanical power input. This machine has been fitted with a small diagonal-component SQUID gradiometer which shows, in a preliminary way, that SQUIDs and refrigerators can be successfully integrated. For demonstration purposes this gradiometer-cooler system has produced a magnetocardiogram of modest clarity [11]. This combination was also used to investigate the level and source of magnetic interference generated by the cryocooler. Some preliminary work has also been done on a pulsed 
technique for measuring the thermal characteristics (thermal conductivity and specific heat) of the plastic regenerator materials used in these machines [12]. This method is useful because the usual measurement methods are cumbersome due to the very long thermal response times for these materials.

The environmental requirements for a SQUID magnetometer are extreme and, as far as we know, no attempt to operate a SQUID with a commercial cryocooler has been made. The expected vibration and magnetic noise levels are so large that the SQUID output would be meaningless [13]. This is not surprising since the SQUID has a sensitivity of the order of $10^{-10}$ times the earth's magnetic field. Thus, even ignoring the direct magnetic interference from moving parts in the refrigerator, a motion of only one microradian in the earth's field causes an interference output which is $10^{4}$ times the sensitivity. From these figures one readily appreciates the need for vibration reduction. Low speed and low-power operation can produce such reduction. In fact, we have taken the view that optimization of refrigerator design for this purpose is really a minimization of the refrigerator power input. The assumption is that the net interference will scale with the size and power requirement of the drive machinery. The calculations in Section 4 are based on this design philosophy. Obviously, one must also avoid the use of magnetic components and even good conductors (since motion of a conductor in a field produces field variation through induced eddy currents). Even ostensibly non-ferro-magnetic materials have susceptibilities and remanent moments which can be troublesome. Thus, material selection is important. It is not essential, however, to eliminate all interference, since the major interference component is synchronous with the drive system of the refrigerator. Synchronous filtering can thus be used to reduce interference, but it is certainly best to start with a low level of interference because such filtering can never be complete.

Three final points should be made. First, if refrigerators are to become important for the support of superconducting devices, then it is essential that their complexity and cost be not much greater than that of the devices they support. This places some sort of bound on practical solutions to the problem. Second, there is a possibility that high- $T_{c}$ devices can alter the situation. That is, the cooling requirements are significantly affected by use of devices that might operate at say 10 or $15 \mathrm{~K}$. Fabrication technology for these devices is not highly developed, but good devices have been reported by several laboratories, perhaps most notably the Naval Research Laboratory. Where such devices could be used, the size and cost of the cryocooler would be reduced significantly [14]. Finally, the feature of most commercial cryocoolers which contributes to the interference is an unnecessarily large refrigeration capacity and drive power (of the order of kilowatts). By matching the crycoooler capacity to the inherently low refrigeration requirements of a SQUID magnetometer, a large reduction in drive power and direct magnetic interference from the drive train can be realized. Cost can be reduced and portability enhanced at the same time. This is really just a restatement of the design philosophy mentioned in the previous paragraph.

\section{Background}

\section{1 Split Stirling-Cycle}

The operation of a split Stirling-cycle refrigerator can be understood through study of figure 1 . Gas moves freely by the displacer exchanging heat with the displacer and walls as it moves between the hot end $\left(T_{1}\right)$ and cold end $\left(T_{2}\right)$. Thus, the pressure throughout the system is nearly the same at any instant in time. Work $W=\int P d V$ may be done on the system as the piston moves back and forth, but no work is done by or on the displacer. When the displacer is at its lowest position, the piston is moved to the left compressing the working fluid (helium gas) and producing heat $Q_{1}$, which is rejected at $T_{1}$. 
Then the displacer is moved to the top of the cylinder so that gas is displaced from top to bottom. Assuming that the machine has been operating for some time and the bottom temperature $T_{2}$ is less than $T_{1}$, then the gas gives up heat $Q_{a}$ to the displacer and wall in the annular gap and arrives at the bottom at a temperature $T_{2}$. The piston then moves to the right producing a heat flow $Q_{2}$ into the gas at $T_{2}$. Finally, the gas is moved upward as the displacer is returned to its bottom position and a heat $Q_{b}$ is added as the gas temperature goes from $T_{2}$ back to $T_{1}$. This completes one cycle of operation.

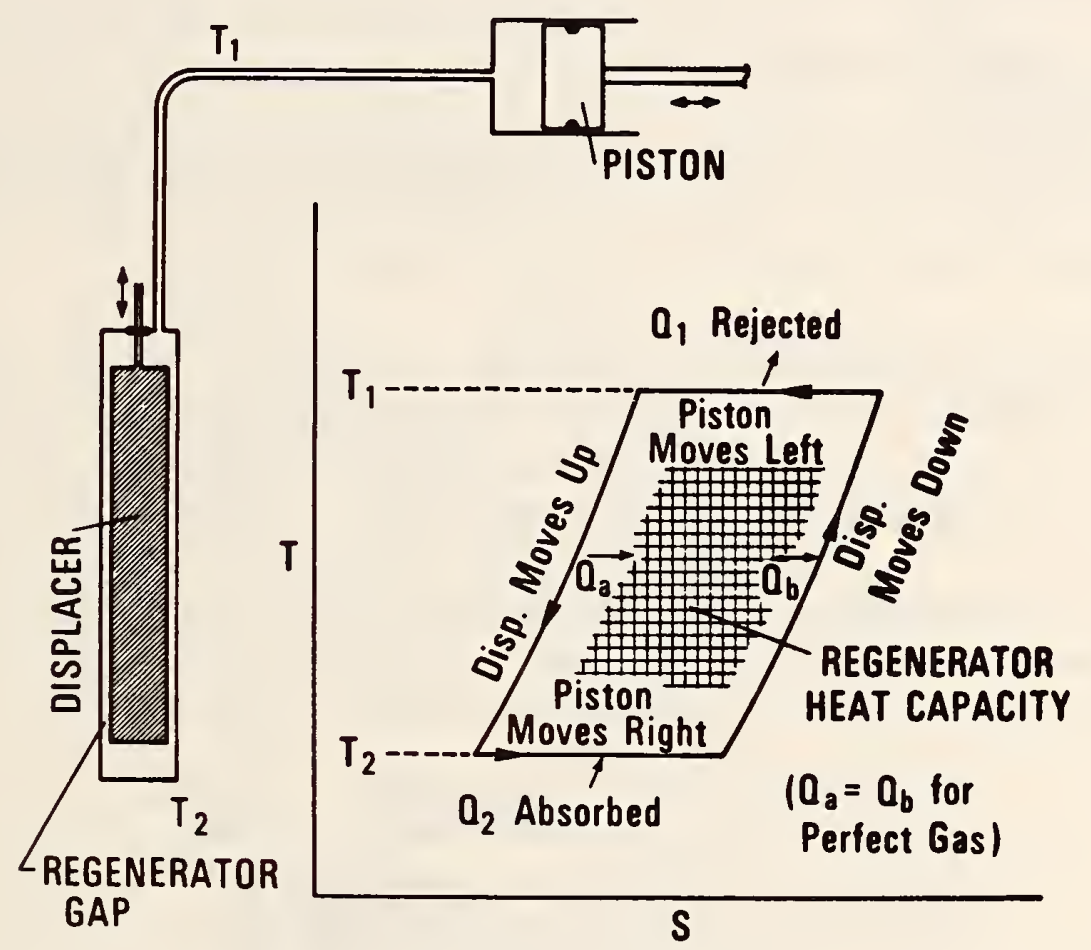

Figure 1. Single-stage, split-cycle Stirling refrigerator with phase diagram to show the idealized thermodynamic cycle.

The process of heat exchange between the gas and the materials in the annular gap is called regeneration. For efficient regeneration the heat capacity of the solid must be much greater than that of the working fluid. This is usually easy to achieve above 10 or $20 \mathrm{~K}$ for helium, but the specific heat of solids relative to helium drops rapidly below this temperature leading to large inefficiency. This, along with the non-ideal behavior of helium near its boiling point generally limits the bottom end temperature in the 5 to $7 \mathrm{~K}$ range. At the sacrifice of cooling capacity one can reduce the helium pressure to enhance the heat capacity ratio. This is the basis for the $3.1 \mathrm{~K}$ temperature achieved by the twin-piston refrigerator [6].

The Stirling-cycle refrigerator described above is readily adapted to multistage operation. A single stage machine such as shown schematically in figure 1 was found to cool to less than $50 \mathrm{~K}$ from room temperature [1]. Figure 2 shows the simple modification which converts the device to multistage operation. At each step in the displacer there is an annular space at which cooling takes place. The successively lower steps (stages) are equivalent to the addition of another refrigerator which rejects its heat to the step above. Concentric radiation shields from each stage enclose all lower stages so that radiation is intercepted and pumped at intermediate temperatures where refrigeration efficiency is high. Further discussion of the split Stirling-cycle refrigerator is contained in references 1 and 10 . 


\subsection{Twin-Piston Stirling Cycle}

We have used the twin-piston Stirling-cycle for only one low-pressure experiment and this work is not currently continuing, so we will not dwell on this particular configuration. Suffice it to say that two pistons operating from the same crankshaft can accomplish the task of shuttling gas back and forth through a regenerator as well as providing the required compression and expansion necessary to approximate the cycle shown in figure 1. This mode of operation proved useful for a low-pressure machine which achieved a temperature of $3.1 \mathrm{~K}[6,8]$.

3. Summary of Refrigerator Development

3. 1 Split Stirling Cycle

In this Section we summarize the construction details and performance characteristics of four coolers which are listed in 3.1 .4 below. The four machines use one, three, four, and five stages achieving temperatures less than $50 \mathrm{~K}, 13 \mathrm{~K}, 8.5 \mathrm{~K}$ and $7 \mathrm{~K}$, respectively.

\subsubsection{Materials}

The four coolers all use nylon as a displacer with spun-glass epoxy (designated G-10 by the National Electronic Manufacturers Association) as the cylinder material. The radiation shields shown in figure 2 are fabricated of thin-wall aluminum, but could equally well be composite fiberglass structures with strips of foil running along their length. The requirement is a reasonable axial thermal conductivity with little or no circumferential eddy currents. The composite shields have been used successfully in many fiberglass dewar systems and should work well here. The aluminum was used here to speed construction. Besides the vacuum insulation, aluminized mylar is used to reduce radiation losses. We have used this material without the usual fibrous spacer.

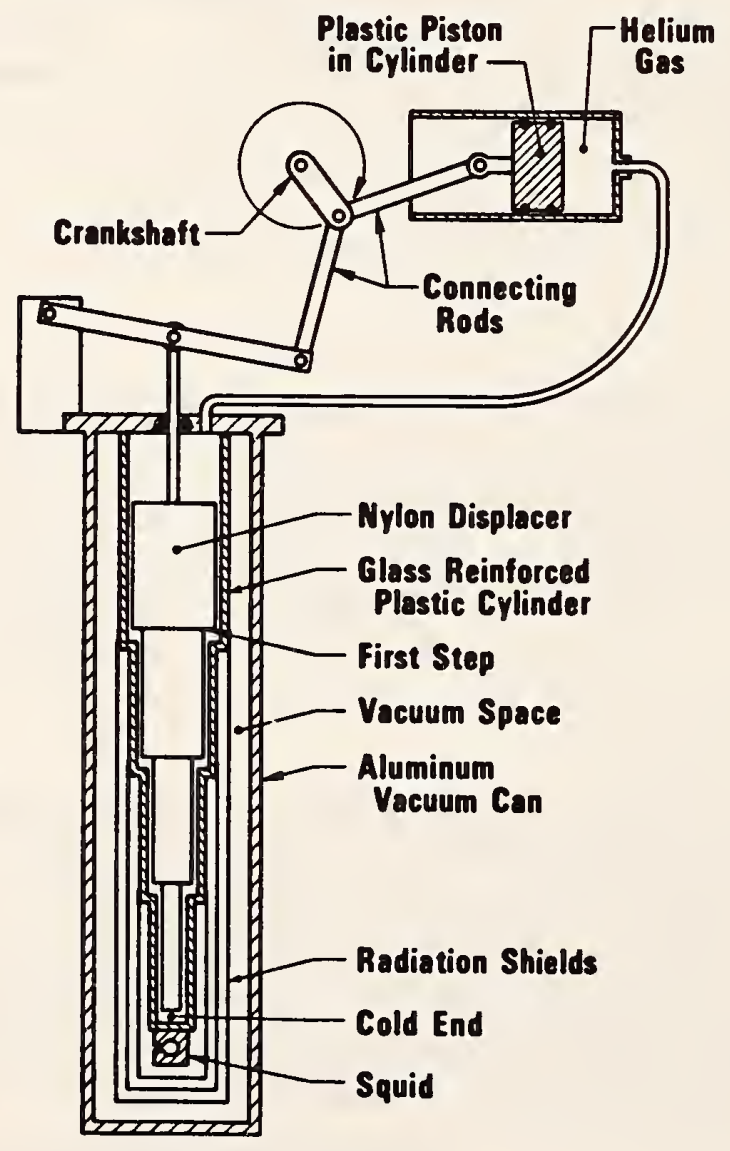

Figure 2. Multistage split-cycle Stirling refrigerator. 


\subsubsection{Construction}

The glass-epoxy (G-10) tubes are purchased from manufacturers stock in a form which is immediately usable. That is, very little machine work is required on these tubes. The tubes are laid up on very precise mandrels so the interior finish and dimensions are well controlled. The exteriors of the tubes are ground and are often not concentric with the inner surface. This is an important fact which must be considered in the assembly of multistep machines. To construct a machine such as shown in figure 3 , the outer end of each section is machined so that the outside part which slips inside the adjacent cylinder is concentric with its interior surface. The connections between cylinders and to the aluminum flange and end cap are all made with epoxy. We have found that it is best to machine these parts so that they mate with an interference fit. Thus, the epoxy is used primarily as a sealant and bond rather than a filler. These joints have been highly reliable.

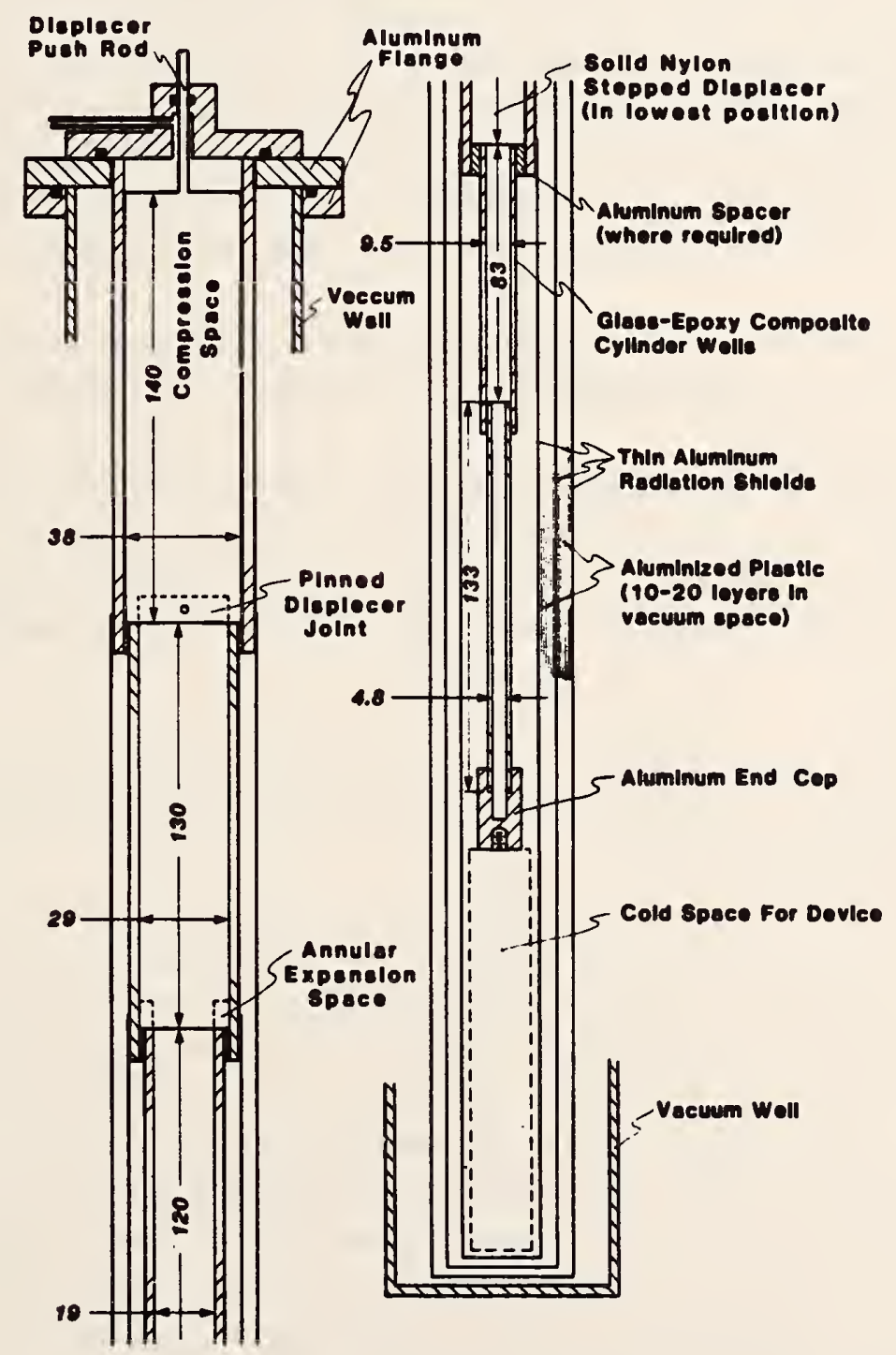

Figure 3. Details of a 5-stage, split-cycle Stirling refrigerator. Dimensions are in millimeters. The drawing is split in two parts to show finer detail. 
The nylon displacer is formed from stock rods which are pinned together to form a multistage machine. Each rod is individually fitted to its appropriate cylinder prior to assembly of the dissplacer or the stepped cylinder. In general, the nylon stock is slightly too large and must be machined to fit the cylinder. These displacer sections are carefully machined to fit the cylinder with almost no radial clearance, except for a few $\mathrm{cm}$ at the large end where about $0.1 \mathrm{~mm}$ radial clearance is provided. During cooldown, differential expansion between the nylon and fiberglass produces a onepercent radial clearance in the lower portions of the refrigerator. This fitting method is simple and minimizes the regenerator gap volume, which is important for achieving lowest temperature.

The differential expansion in the axial direction must also be considered. The lengths of the displacer sections are selected as as to match the lengths of the corresponding sections of the cylinder when the system is at its lowest operating temperature. This requires some foreknowledge of the temperatures of the different stages and an estimate of the differential expansion as a function of temperature. After initial cooldown the displacer is adjusted so that (ideally) all steps reduce their respective volumes to zero at the bottom of the stroke.

Figure 3 provides dimensional information (in millimeters) on the 5-stage cooler. The upper end of each radiation shield is slit so that it can be slipped over the appropriate step on the cylinder and secured with 20 to 40 turns of thin nylon string (unwaxed dental floss is ideal). The bottom ends of these aluminum tubes are closed with aluminum foil or aluminized mylar which is also secured with dental floss.

Mylar superinsulation is used between each shield and between the outer shield and the vacuum wall. We have found that this insulation can be omitted with little effect in the space between the cold end and the first shield.

All instrumentation wiring (thermometer leads, thermocouple wire and $r f$ leads to SQUIDs) are thermally anchored at each stage. In one instance, we failed to fully anchor one pair of leads at the next to bottom stage and found significant deterioration in the bottom end performance. Thermal anchoring is achieved by securing the leads tightly with dental floss and then coating the area with a suitable varnish.

\subsubsection{Operation}

The displacer push rod in figure 3 is fitted with an adjusting screw so that the bottom limit of the displacer motion can be altered as the systen cools down. The range of this adjustment is about one percent of the displacer length.

After purging the system of air (either simple purging with helium gas or pumping the air out), the system is filled with helium to between 2 and 4 bars pressure. The piston and displacer are initially set to $90^{\circ}$ phase difference and the system is put into operation. From this point on there are three adjustments which can be made. These are the phase, the pressure and the displacer spacing from the cylinder bottom. The latter is the simplest, since the adjustment can be made by noting the point where the displacer hits bottom and then backing off slightly from that position. The adjustment of pressure and phase for lowest bottom end temperature is generally different from that for fastest cooldown and the selection of proper parameters for either optimization is tedious since the system responds very slowly. It is quite easy to be misled in these adjustments. For example, in optimizing for lowest temperature one often finds that a small adjustment in phase or pressure results in a downward trend in temperature, but this trend is only a transient and the final steady state can, in fact, be higher than the initial temperature. This behavior follows from the fact that these parameters affect every stage of the refrigerator and the upper stages respond slowly because of their higher total heat capacities. For the 5-stage machine, we have found that the optimum pressure is between 2 and 2.5 bars (gauge pressure) and the phase is closer to $45^{\circ}$ than $90^{\circ}$. 
With the sliding ' 0 ' ring seals on the displacer push rod and in the compressor (see Section 3.3), there is a very gradual buildup of air contamination in the system. This freezes into the lower parts of the displacer and cylinder and gradually causes friction and sticking of the displacer with a resultant increase in temperature. The system must be warmed up and purged of these contaminants before recooling. Presently this problem occurs every one to three weeks depending upon the quality of the sliding seals. With further engineering work this problem should be eliminated.

A second problem results from the fact that helium diffuses through the spun glass-epoxy material. Thus, the vacuum space must be continuously pumped to maintain the integrity of the insulation. The pump can be turned off for only short periods ( $15 \mathrm{~min}$. to an hour) before the temperature starts to rise. In principle, this problem could be resolved by laminating a thin metal foil into the glass-epoxy cylinder to block the diffusion.

\subsubsection{Discussion and Summary of Split Stirling Refrigerators}

In table I we summarize the characteristics of the four machines which have been built and tested. The one-stage cooler was really operated with a compressed gas cylinder and valves, that is, in a Gifford-McMahon cycle, although it could easily have been converted to Stirling cycle. The bottom end temperature would probably have been about the same. Measurement and modeling of shuttle heat loss [4, 5] indicate that such loss can be reduced by shortening the displacer stroke. This is the reason for the shorter stroke in the 4 and 5 stage machines.

TABLE 1

CHARACTERISTICS OF FOUR MULTISTAGE CRYOCOOLERS

\begin{tabular}{|c|c|c|c|c|c|}
\hline \multicolumn{2}{|c|}{ Lowest Temperature } & $50 \mathrm{~K}$ & $13 \mathrm{~K}$ & $8.5 \mathrm{~K}$ & $7 k$ \\
\hline \multicolumn{2}{|c|}{ Compressor Displacement (cc) } & * & 38 & 38 & 90 \\
\hline \multirow{5}{*}{$\begin{array}{l}\text { Diam./Length - } \\
\text { (both in } \mathrm{mm} \text { ) }\end{array}$} & Stage 1 & $9.6 / 300$ & $19 / 245$ & $28 / 150$ & $38 / 140$ \\
\hline & Stage 2 & & $9.45 / 143$ & $19 / 120$ & $29 / 130$ \\
\hline & Stage 3 & & $4.7 / 144$ & $9.5 / 100$ & $19 / 120$ \\
\hline & Stage 4 & & & $4.7 / 150$ & $9.5 / 83$ \\
\hline & Stage 5 & & & & $4.8 / 133$ \\
\hline \multicolumn{2}{|l|}{ Stroke $(\mathrm{mm})$} & 12.7 & 12.7 & 7 & 7 \\
\hline \multirow[t]{5}{*}{ Temperature - } & Stage 1 & $50 \mathrm{~K}$ & $120 \mathrm{~K}$ & $180 \mathrm{~K}$ & $167 \mathrm{~K}$ \\
\hline & Stage 2 & & $40 \mathrm{~K}$ & & $70 \mathrm{~K}$ \\
\hline & Stage 3 & & $13 \mathrm{~K}$ & & $27 \mathrm{~K}$ \\
\hline & Stage 4 & & & $8.5 \mathrm{~K}$ & $15.5 \mathrm{~K}$ \\
\hline & Stage 5 & & & & $7 \quad k$ \\
\hline
\end{tabular}

*This machine was operated with valves by compressed gas (i.e., Gifford-McMahon cycle). 
The addition of more and more stages leads one closer to a continuously tapered displacer, and we now believe that this might in fact be the next thing to do. The construction will require some custom fabrication of the displacer and cylinder, but we suspect this may really be simpler than the present construction which requires a lot of careful fitting and joining of stock tubes and rods. Another point to make is that a tapered displacer model can really offer a fair approximation to a stepped displacer machine. In section 4 we consider such a model and find that it describes the stepped displacer results quite well.

\subsection{Twin-Piston Stirling Refrigerator}

This development is described rather completely in the paper by Zimmerman and Sullivan [6] with some additional discussion in reference [8]. Thus we omit extensive description of the device here. We will emphasize only a few points which are not made in those papers.

The idea behind this machine was to gain lower temperature in a single low-temperature stage. By reducing the helium pressure to values below atmospheric, one lowers the boiling point of the liquid and thus maintains nearly ideal gas behavior until this lower temperature is approached. More importantly, the ratio of regenerator heat capacity to gas heat capacity is enhanced and improves the regenerative action.

Operating from upper temperatures of 8 to $12 \mathrm{~K}$ the machine achieved bottom end temperatures of 3.1 to $4 \mathrm{~K}$ as the average pressure was varied from 0.26 to 0.79 bars. In all cases, a small amount of gas was liquified in the bottom of the cooler, and the temperature achieved was just the boiling point of helium at the average operating pressure, e.g. $3.1 \mathrm{~K}$ at 0.26 bars. To our knowledge, this is the first Stirling refrigerator in which such liquefaction has been demonstrated.

The twin-piston machine described in reference [6] used epoxy fiberglass for all the lower end components, so there was no need for concern about differential expansion. The two pistons were set to a $90^{\circ}$ phase difference and no further phase adjustments were made. Such adjustment would have been difficult because the crankshaft and connecting rods were sealed into a helium-filled enclosure.

This device could be coupled to one of the split Stirling machines to achieve helium temperature operation in one system, but it must be operated as a completely separate machine because of the low operating pressure. The complication of combining two refrigerators has caused us to concentrate on further improvement of the split Stirling-cycle refrigerator in the hope that this machine will prove suitable for most applications.

\section{3 Compressor Design}

We report here on the details of construction of three different compressors, a simple ' 0 ' ring sealed piston with conventional crosshead, a balanced force device, and a ceramic oil-free system.

\subsection{1 ' 0 ' Ring Sealed Piston with Conventional Crosshead}

The simplest compressor was constructed with a nylon piston having one or two ' 0 ' rings for seals with a felt pad loaded with silicon grease in an adjacent groove to provide lubrication. A sketch of such an arrangement is found in reference [1]. This piston was driven at approximately 1 stroke per second by a motor and crosshead made up of an old commercial freon compressor. The $38 \mathrm{cc}$ compressor had a piston diameter of $35.6 \mathrm{~mm}$ and a stroke of $38 \mathrm{~mm}$. The $90 \mathrm{cc}$ compressor used a stroke of $51 \mathrm{~mm}$ with a piston diameter of $48 \mathrm{~mm}$. 


\subsubsection{Force Balanced Compressor}

There are two problems with the simple compressor. First, the force needed for compression requires a rather large motor. Much of the work done in compressing the gas can be recovered during the expansion phase. Thus it is possible to balance this force with other conservative forces so as to reduce the torque needed to operate the compressor. The first and simplest modification is to keep helium gas on the back side of the piston at a pressure which is the average value of the system pressure. This leaves the system at equilibrium with the crankshaft $90^{\circ}$ ahead or behind full compression which reduces the required torque. Further reduction can be accomplished through the use of a spring and cam as shown in figure 4. An added benefit is that the piston ' 0 ' rings are not directly exposed to the atmosphere. Air leakage is only through the seal around the piston rod.

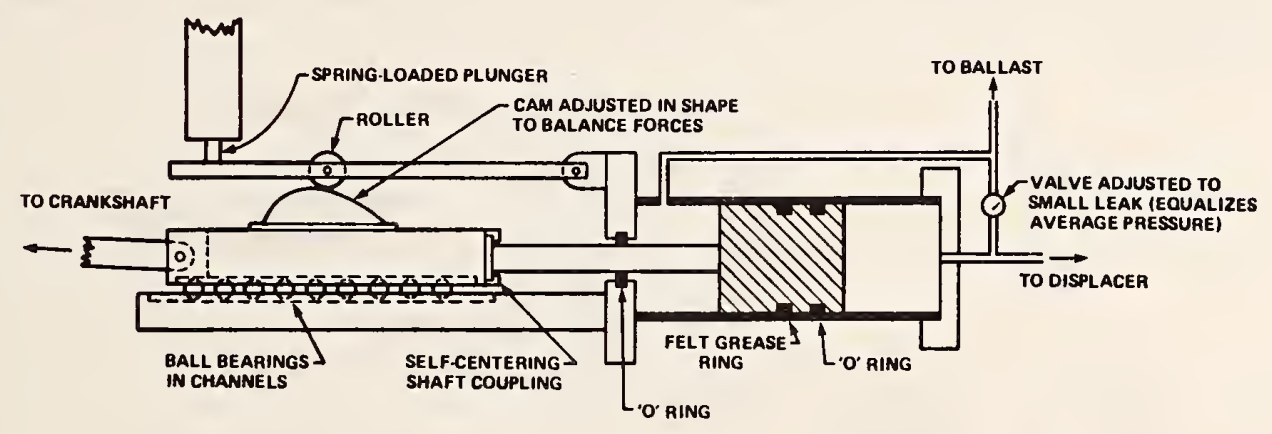

Figure 4. Sketch of mechanism used to balance the compressor. The spring and cam are adjusted and shaped so that the torque at the crankshaft is nearly constant.

\subsubsection{Contamination Free Compressor}

Ultimately one should address the problem of a design which has no lubricated seals and no possibility for buildup of air contamination. While the latter has been our more immediate problem, it is clear that oil-free operation is essential for continuous, long-period use. Over a long period of time oil vapor would build up deposits in the cold portion of the refrigerator with resultant deterioration in performance. There are several approaches to the design of a contamination-free compressor, and while this is not the major thrust of our effort, we describe here some preliminary experience with one concept.

The compressor shown in figure 5 uses very closely spaced ceramic (alumina) cylinders to effect a seal during compression. The radial clearance of 4 micrometers allows very little pressure loss during compression and the back side of the piston is sealed with a metal bellows to complete the gas containment. The time constant for pressure equalization across the piston is of the order of one minute. Thus, leakage past the piston is negligible at the operating speed of one stroke per second. This compressor was operated approximately 60 hours at which point the bellows failed. Subsequently, we replaced the bellows with an ' 0 ' ring seal. This system was run non-stop for over 10 months. The results of this test indicate some initial wear but little or no wear after about 1000 hours. The wear is inferred from measurement of leak rate past the cylinder (see figure 6). There is some discrepancy in the measurements beyond 3000 hours probably due to miniscule accumulation of contaminants on the surfaces. The measurements at 7100 hours showed a small increase in leak rate when the surfaces were cleaned with toluene. 


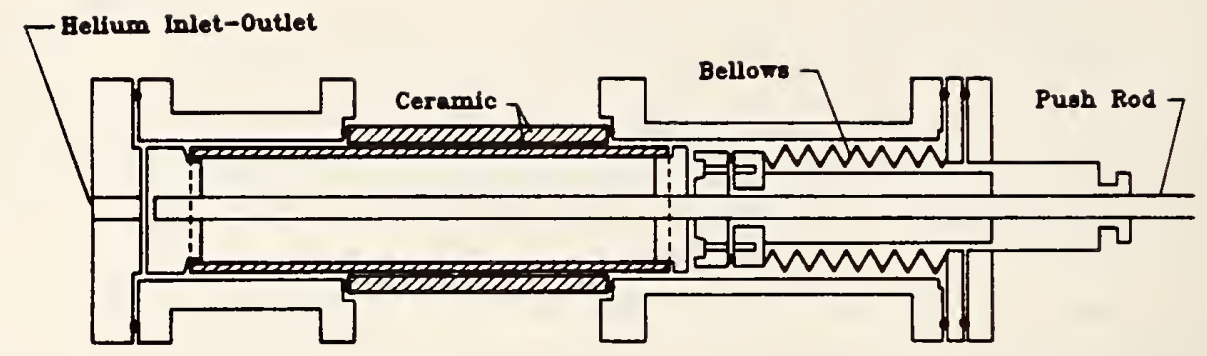

Figure 5. Details of design of an oil-free compressor. The clearance between ceramic components is 4 micrometers keeping gas leakage at a negligible level during compression. The compressor was actually operated vertically.

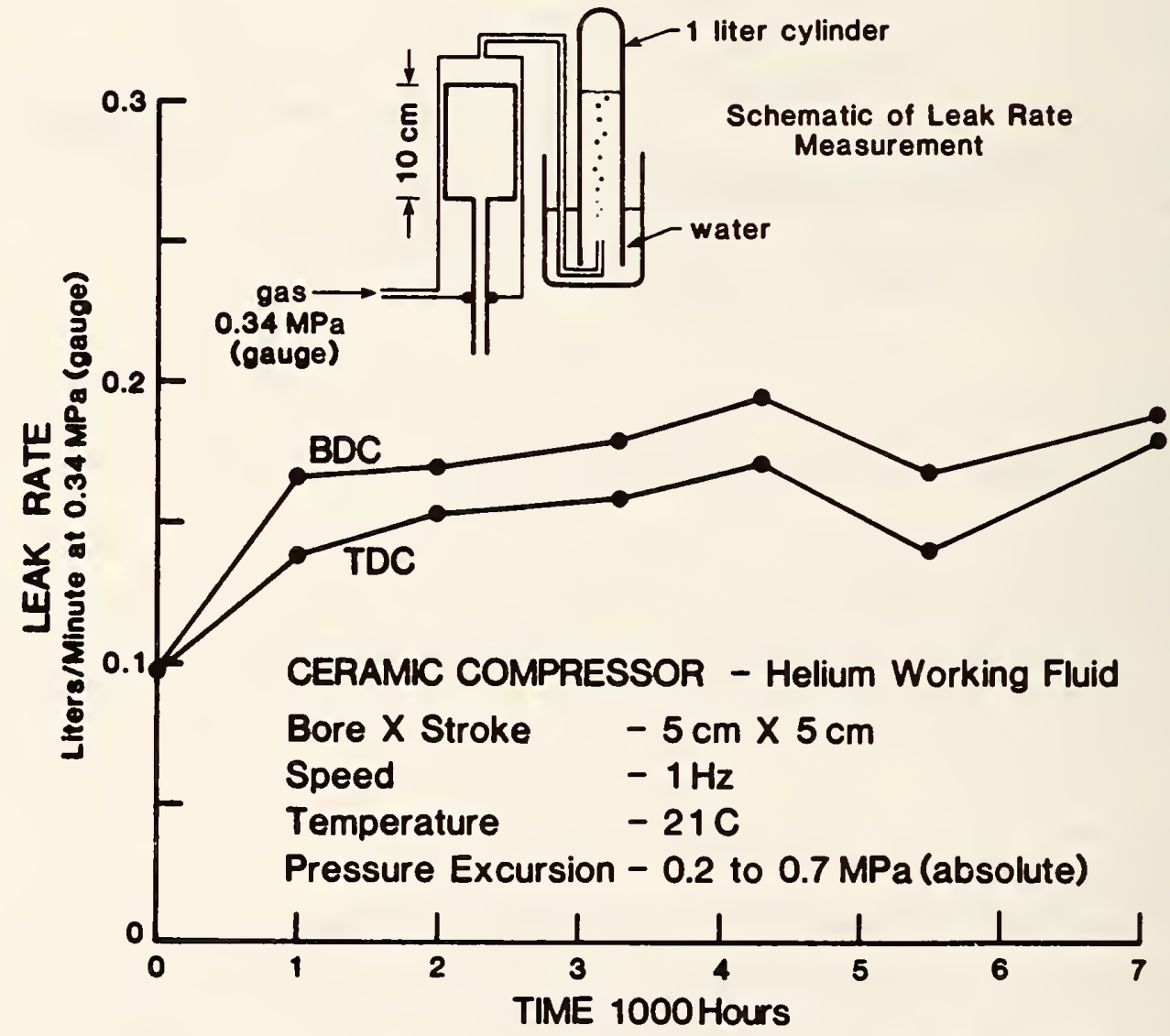

Figure 6. Wear measurement for the ceramic compressor. Wear is inferred from change in leak rate with time. The experimental setup is shown in the insert at top. BDC and TDC stand for bottom dead center and top dead center, respectively.

\subsection{Temperature Control}

In abandoning the use of liquid helium in favor of a miniature cryocooler, it, is necessary to take measures to stabilize the temperature of the cold end of the cryocooler. We have observed that small variations in operating parameters (such as ambient temperature, speed, pressure, impurity buildup, etc.) cause significant slow variations in cold-end temperature, of the order of $0.1 \mathrm{~K}$ or more. Also, we see a temperature oscillation at the cryocooler frequency $(1 \mathrm{~Hz})$. This oscillation is probably 
quite large in the expansion chamber itself, but may be quite small at the superconducting device, depending on the thermal resistance between it and the expansion chamber, and on the thermal mass of the device and associated hardware. In the four-stage cryocooler, the amplitude of the oscillation was about $2 \mathrm{mK}$ at $8.5 \mathrm{~K}$, as measured by a carbon resistance thermometer cemented into a brass block screwed to the bottom of a helium gas thermometer bulb clamped to the cold end of the cryocooler (see figure 7). The amplitude measured by the gas thermometer was about $5 \mathrm{mK}$. It is to be expected that the amplitude of temperature oscillation should be larger, the closer the thermometer is to the expansion space. It will also, of course, be a function of the response time of the thermometer.

In order to stabilize and control the temperature of the brass block (which simulates a SQUID with mounting hardware) shown in figure 7 , we assembled the simple circuit shown in figure 8 to amplify the dc-signal from the carbon resistance thermometer and feed it back to the 10,000-ohm resistance heater. Thus if the control resistance $R_{C}$ in the bridge is set lower than the thermometer resistance $R_{T}$ with feedback loop open, the closed-loop output of the amplifier will provide power to the heater $R_{H}$ to raise the temperature of the brass block until the bridge is balanced. Initial tests of this control system showed that slow changes in temperature become imperceptible (no more than a fraction of a $\mathrm{mK}$ in a two-hour period). Because of the rather slow thermal response, the simple circuit did not significantly reduce the $2 \mathrm{mK}$ oscillation at $1 \mathrm{~Hz}$. We intend to work on electronic regulation of the oscillatory component in the future.

Another method for stabilizing the temperature is to increase the heat capacity of the object attached to the cold end. This might best be done with helium gas in a small volume between the cold end and the SQUID. The situation would be like that of figure 7 . We found that the heat capacity of this system could easily be increased by a factor of 10 or more by filling the 1 cc volume to only 4 or 5 bars of pressure. This results in a 10-fold reduction in the oscillatory component of temperature variation. This also reduces the drift rate significantly.

This latter method of temperature stabilization can readily be developed further. The concept is analagous to electronic filtering with resistor-capacitor combinations. The heat capacity of an object is the analog of electrical capacitance and thermal resistance is the analog of electrical resistance. Using this analog, one realizes that thermal resistance should be introduced between the refrigerator and gas reservoir to improve the filtering.

4. Cryocooler Optimization

4. 1 General Approach

The general design philosophy for these cryocoolers involves one fairly simple assumption; to minimize refrigerator interference one should operate with the smallest possible drive power. In other words, the larger the drive machinery, the greater will be the magnetic and vibrational interference for any SQUID or other system supported by the refrigerator. Thus, when we say optimum design, we mean minimization of the power requirement for the system.

We have selected a tapered displacer Stirling cooler for this calculation with the following thoughts in mind. First, we can cover the entire temperature range from just above liquid helium temperature to room temperature with a single calculation. Second, the results will provide design guidance, even for stepped displacer machines. That is, the result of the calculation is an optimum shape and size for the displacer and this can be used to estimate the lengths and diameters of the stages in an optimum stepped displacer machine. Finally, we would like to consider the construction of a tapered displacer system in the future.

The idea in this calculation is to express the refrigeration and losses as time-averaged functions of the parameters of the system (heat capacity, thermal conductivity, position along the displacer, 
stroke, frequency, etc.). The losses are balanced against the refrigeration with the functional form of the taper (cylindrical symmetry) finally determined by using a variational method to minimize the power required to run the system.

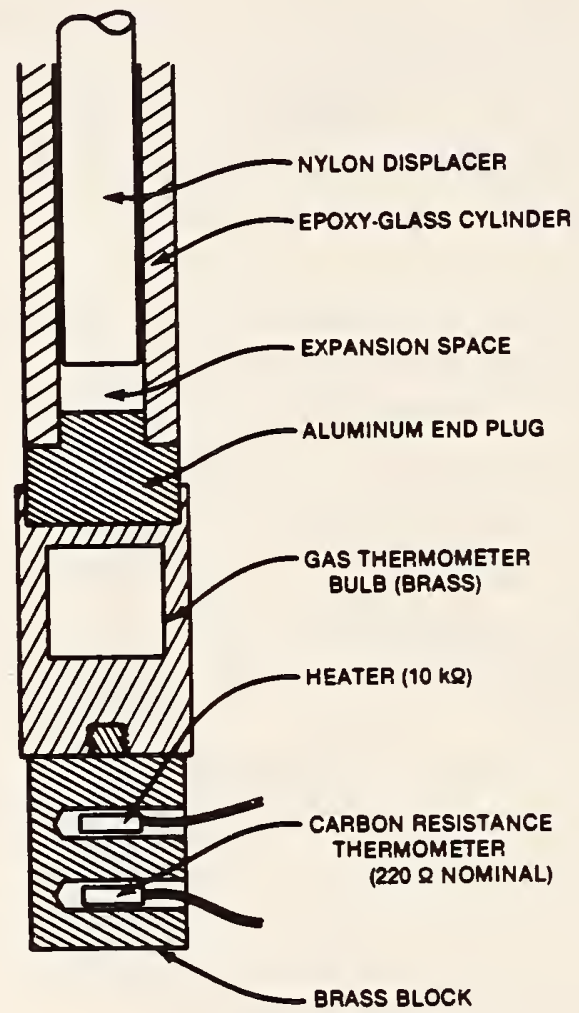

Figure 7. Bottom-end details of the 4-stage cooler with electronic temperature control. Helium gas in the bulb adds significant thermal mass and thus adds to temperature stability.

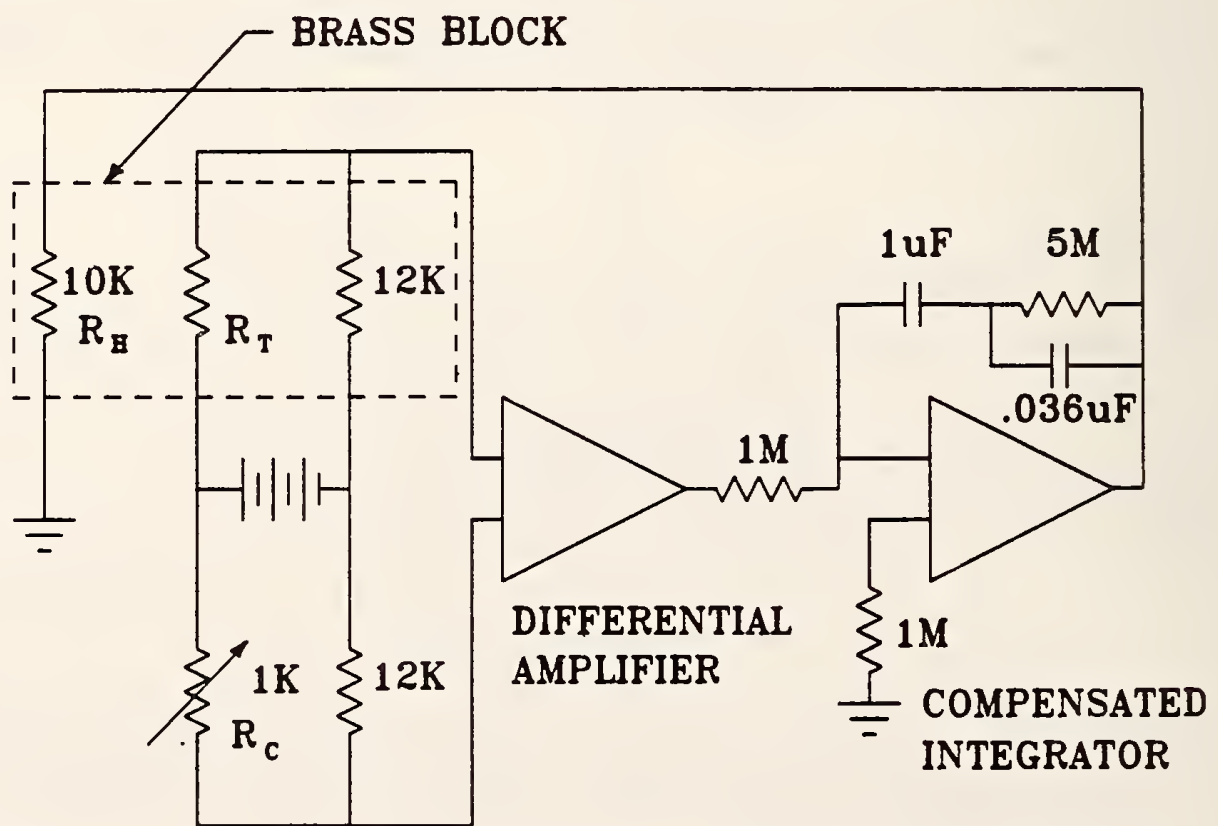

Figure 8. Simple temperature control bridge circuit. The amplifier delivers current to the heater so as to match $R_{T}$ to $R_{C}$. 


\subsection{The Calculation}

In this calculation we consider three loss terms; conduction, radiation, and shuttle heat loss. These are the dominant losses for the temperature range of about 15 to $300 \mathrm{~K}$. Below $15 \mathrm{~K}$ one should include the enthalpy deficit and regenerator inefficiency. These terms are more complex because they include the mass flow of the working fluid. Thus, as a first step we consider the limited problem which nonetheless results in useful design information in the temperature range above $15 \mathrm{~K}$. Despite the potential for some error below $15 \mathrm{~K}$, we have extended the calculations to $10 \mathrm{~K}$.

The net heat leak can be expressed as

$$
\begin{gathered}
\dot{Q}=\dot{Q}_{\text {cond }}+\dot{Q}_{\text {rad }}+\dot{Q}_{\text {shuttle }} \\
=\pi r^{2} k(d T / d x)+2 \varepsilon \sigma T^{3} A f(x)(d T / d x)+s^{2} r(\pi \dot{n} k C / 2)^{1 / 2}(d T / d x),
\end{gathered}
$$

where $k$ is thermal conductivity and $C$ is specific heat, both of which are functions of $x$. $r$ is the radius, $S$ is the stroke, $A$ is the surface area of the radiation shields, $n$ is the operating frequency, and $\varepsilon$ and $\sigma$ are constants relating to radiation. $f(x)$ is a function which describes the distribution of insulation along the refrigerator. $x$ is the position along the displacer and the radius $r$ is taken as a general function of $x$, so that the taper profile is as yet unspecified. The third term, shuttle heat loss is taken from reference [4].

The thermal conductivity and specific heat of the displacer and cylinder are functions of temperature and must be explicitly included in the calculation. From a combination of sources including reference [4], we have generated the following power series representations of thermal conductivity, $k(T)$, and specific heat, $C(T)$ for the $G-10$ material in the range from $5 \mathrm{~K}$ to $300 \mathrm{~K}$.

$$
\begin{aligned}
& k(T)=9.17 \times 10^{-2}+3.19 \times 10^{-3} \mathrm{~T}-3.67 \times 10^{-6} \mathrm{~T}^{2}, \\
& C(T)=-1.49 \times 10^{4}+3.75 \times 10^{3} \mathrm{~T}+3.35 \times 10^{1} \mathrm{~T}^{2}-9.21 \times 10^{-2} \mathrm{~T}^{3},
\end{aligned}
$$

where $k(T)$ is in units of $\mathrm{W} / \mathrm{K} \cdot \mathrm{m}$ and $\mathrm{C}(\mathrm{T})$ is in $\mathrm{J} / \mathrm{K} \cdot \mathrm{m}^{3}$.

The diameter at the bottom end of the displacer is set by equating the refrigeration at the bottom end with the heat leak into the same point, that is,

$$
\dot{Q}\left(r_{0}\right)=\pi r_{0}^{2} \sin P_{u} \ln \left(P_{u} / P_{\ell}\right)
$$


where $r_{0}$ is the bottom end radius and $P_{u}$ and $P_{\ell}$ refer respectively to the upper and lower pressures. The expression on the left is the sum of the heat losses (eq. 1) evaluated at $r=r_{0}$ (or equivalently, at $x=0$ ). The refrigeration term is an ideal one; it should be reduced by about one half for the real sinusoidal drive.

The ideal work required by the refrigerator is given by

$$
W=\int\left(T_{a m b} / T-1\right)(d \dot{Q} / d x) d x,
$$

where the integral is over the length of the machine. The first term inside the integral is the Carnot coefficient and $T_{a m b}$ is the ambient (room) temperature.

Equation 4 can be solved for $r_{0}$ as a function of $T_{0}$ (the bottom temperature) and $(d T / d x)_{x=0}$. Thus if we select an arbitrary function, $T(x)$, to describe the temperature profile, we can calculate a value for $r_{0}$ and then, having differentiated $\dot{Q}$ with respect to $x$, we can calculate the net work (eq 5 ) for this temperature profile. Equation 4 can also be used to find $r$ as a function of $x$; since the heat flow at a point, $x$, is intercepted by the refrigeration below that point to the displacer length.

The results described are for a specific $T(x)$ and thus do not represent any sort of optimum, but we do have all of the ingredients for a variational determination of the optimum $T(x)$ and thus displacer shape. We select a power series for $T(x)$,

$$
T(x)=T_{0}+T_{1} x+T_{2} x^{2}+T_{3} x^{3}+\ldots . .
$$

where the sum of the coefficients, $T_{n}$, is equal to $T_{\text {amb }}{ }^{-T_{\text {bottom }}}$ if $x$ is normalized to the displacer length. An arbitrary set of coefficients (usually only a linear term) is chosen and after calculating the work, the coefficients are varied to find if this increases or decreases the work and only changes which decrease the net work are accepted. By successive iterations of this process for all of the coefficients we converge upon the best possible temperature profile and displacer shape. The absolute length of the displacer is an input parameter.

\subsection{Results}

Figure 9 shows the results of this computer generated solution for a 50 centimeter long displacer which is comparable to that used in our 4 and 5 stage machines. The stroke is $5 \mathrm{~mm}$, the cylinder wall thickness is $3 \mathrm{~mm}$, and the frequency is $1 \mathrm{~Hz}$. The 4 radiation shields are taken to have an average diameter of $10 \mathrm{~cm}$ and 40 layers of aluminized mylar are distributed between these shields. The upper and lower pressures are 6 and 3 bars, respectively. These parameters are consistent with the values for the 4 and 5 stage coolers described in section 3 . The net work (ideal) required in this refrigerator calculation ( $10 \mathrm{~K}$ bottom end temperature is 0.564 watt with 0.211 watt going to conduction loss, 0.214 watt to shuttle heat loss, 0.082 watt to radiation loss, and 0.057 watt required to cool the bottom end. The bottom end term is primarily shuttle and conduction heat flow which is proportional to the temperature gradient and the cross-sectional area at the bottom end. It would be zero if the wall thickness and displacer radius could be tapered to zero, but this is obviously impractical. Our choice of a constant $3 \mathrm{~mm}$ wall thickness over the whole length was arbitrary, but the 
variational method would work for any functional dependence of wall thickness on axial position. Since our refrigerators require tens of watts of input power, we conclude that most of that power goes into compressor losses (non-isothermal operation), motor losses, and friction.

The distribution of refrigeration to the different loss mechanisms is noteworthy. With modest effort, radiation loss is relatively small. Conduction and shuttle heat loss are comparable. The quantitative agreement between theory and experiment is also shown in figure 9. The stepped displacer radius should be compared with the tapered displacer radius. The agreement is remarkable if three facts are considered. First, the comparison is between a refrigerator with a stepped displacer and a model with a continuously tapered one. Second, in the experiments the parameters were adjusted to achieve the lowest temperature which may not be obtained for the set of parameters which yield the minimum drive power. Third, the temperature achieved was $8.5 \mathrm{~K}$ rather than $10 \mathrm{~K}$.

The only serious discrepancy between analysis and experiment is in the mechanical power required to run the compressor. We have always estimated that the order of 10 watts or more is required, whereas the analysis (see above) gives the order of 0.6 watt. To resolve this discrepancy, we recently measured the pressure-volume ( $P-V)$ diagram of the 4-stage machine (see table 1 ) with the average pressure adjusted to give a steady-state bottom-end temperature of $10 \mathrm{~K}$. The area of the $P-V$ diagram multiplied by the operating speed gave 2 watts as the mechanical power actually performed on the working fluid. Correcting for non-isothermal compression reduced this to a net power of 1.3 watts. In comparing this with the analytical optimum of 0.6 watts, it should be noted again that a stepped displacer is not expected to be as efficient as a continuously tapered displacer, and furthermore the experimental machine had about twice the diameter of the analytical model.

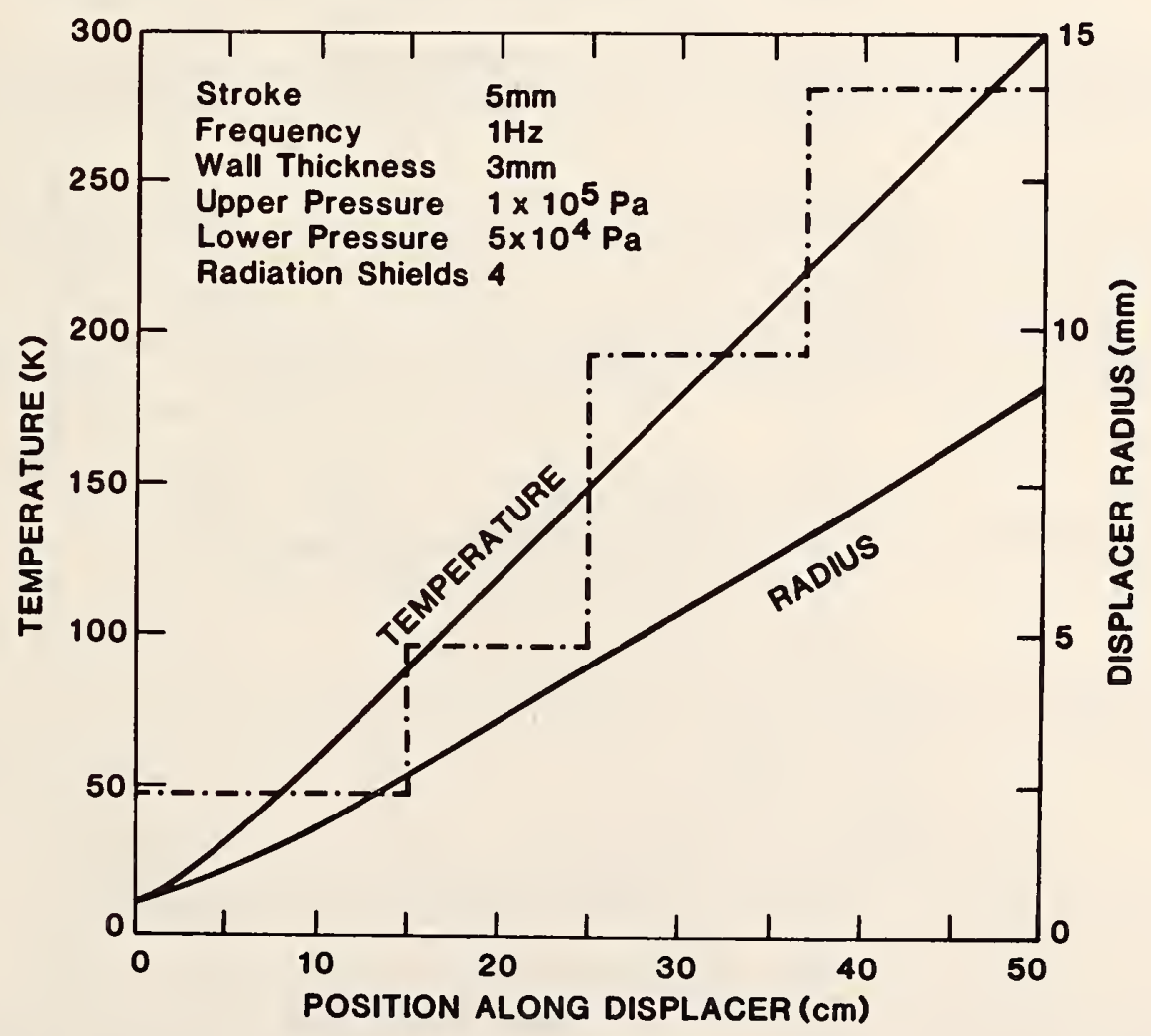

Figure 9. Temperature and displacer radius as a function of position along the radius. This is the optimum shape described by the model for the set of parameters listed above. The stepped displacer for the 4-stage machine is superimposed for comparison with the radius of the tapered displacer. 
With this calculation as a reference, we successively varied the stroke, the length, and the frequency to see whether better values could be predicted. The results are shown in figure 10 . While the length of a half meter was selected somewhat arbitrarily, the stroke and frequency were varied experimentally to arrive at the values given by the circles in figure 10 . It is interesting to note how closely the calculation predicts the best set of values for these parameters. The optimum stroke of $\sim 3.5 \mathrm{~mm}$ is close to the value of $5 \mathrm{~mm}$ used in the refrigerators, and while the minimum power occurs for a displacer length of one meter, the half meter value which is more practical does not produce too dramatic a rise in system power. As for the frequency, it is clear that one should operate at the lower end of the flat portion since the inclusion of regenerator efficiency will probably cause the right end of the curve to be lifted. The gradual rise in power requirement with displacer length reflects the increase in radiation loss which is itself quite small.
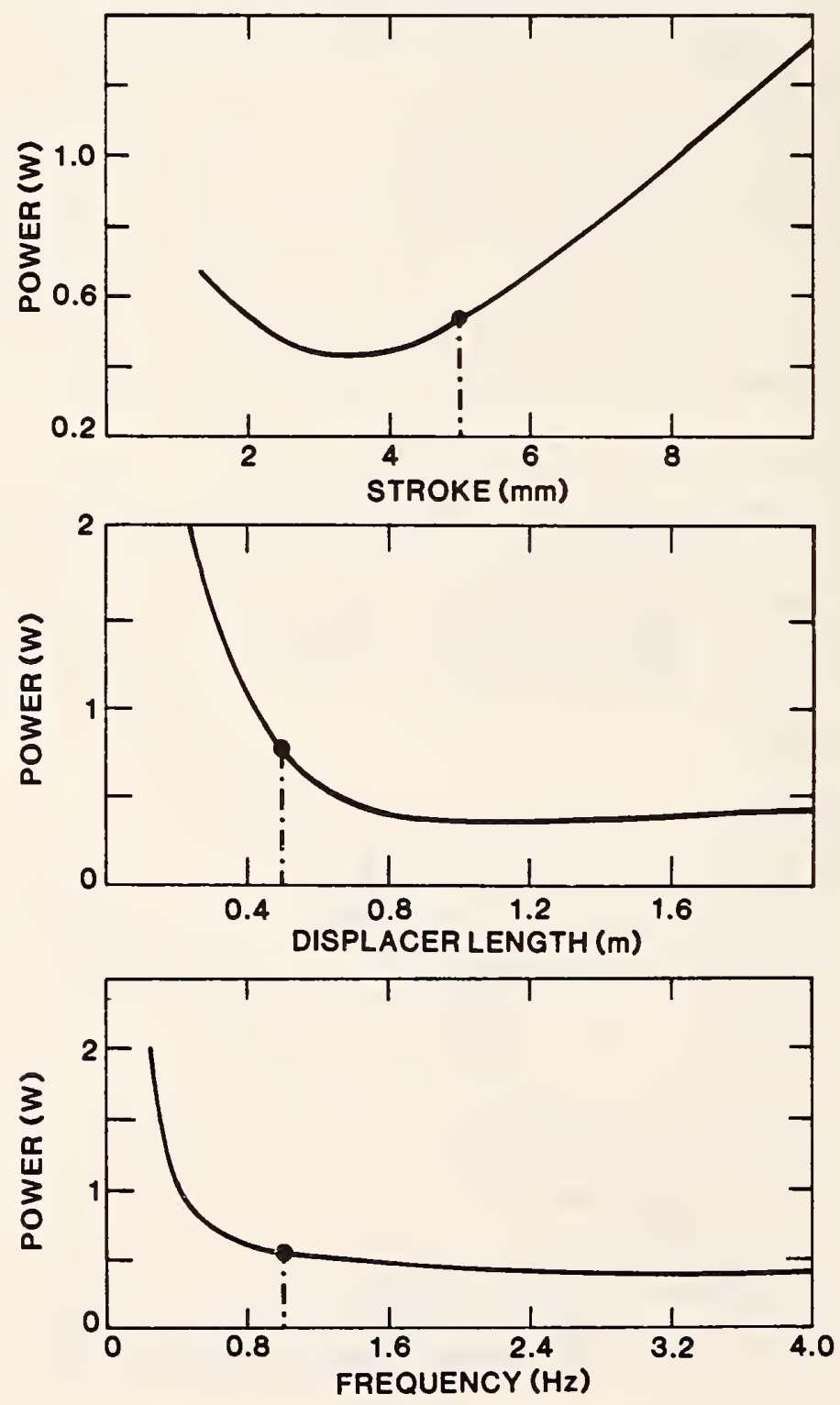

Figure 10. Power requirement as a function of stroke, speed, and displacer length. Each of the 3 parameters is varied separately with the remaining parameters held at the values given in figure 9. 
One can also account for excess dissipation by some apparatus at the bottom of the refrigerator. For the reference conditions (parameters) described above we note a linear increase of require drive power, $35.7 \mathrm{~mW}$, for each $\mathrm{mW}$ of refrigeration at $10 \mathrm{~K}$ for power dissipations of up to $20 \mathrm{~mW}$. There is, of course, a commensurate increase in the diameter of the bottom end of the displacer.

5. Summary and Conclusions

It now appears that this new class of low-power Stirling cryocoolers can be compatible with superconducting sensors, at least for modest sensitivity. Several engineering problems remain to be solved. First, the compressor needs to be designed for dry operation to eliminate any contact between the helium gas and oil. This will require some effort, although the low speed and low working pressure offer possibilities for simple solutions. The problem of helium diffusion through the displacer cylinder should be eliminated with some sort of diffusion barrier. Finally, considerably more work should be done to isolate and identify magnetic interference mechanisms. This could permit extension of the devices to more demanding applications.

All things considered, we feel that the analytical results agree remarkably well with the experimental measurements, insofar as valid comparisons can be made. Thus, the tapered displacer model seems to provide reasonable guidance for refrigerator design. It would be especially useful to extend the calculations to include regenerator inefficiency and enthalpy deficit. The present calculations suggest that a linear taper (cone) will suffice for temperatures down to $10 \mathrm{~K}$, but we suspect that the extra losses below this temperature will require a more complex taper. In any case, it should prove useful to build a continuously tapered system, since success with this concept could lead to improved performance and simplified construction.

\section{References}

[1] Zimmerman, J. E., Radebaugh, R., and Siegwarth, J. D., in "Superconducting Quantum Interferences Devices and their Applications," (H. D. Hahlbohm and H. Lubbig, eds.), Berlin, Walter de Gruyter (1977), pps. 287-296.

[2] Zimmerman, J. E., Radebaugh, R., and Siegwarth, J. D., "Refrigeration for Small Superconducting Devices," Conf. German Refrigeration Society, Munich (October 1976).

[3] Zimmerman, J. E. and Radebaugh, R., in "Applications of Closed-Cycle Cryocoolers to Small Superconducting Devices," NBS Special Publication SP-508, J. E. Zimmerman and T. M. Flyn, eds.), Superintendent of Documents, U. S. Government Printing Office, Washington, D. C. 20234 (1978). (Also available from the authors), pp. 59-65.

[4] See ref. 3 pps. 67-73.

[5] Radebaugh, R. in "XVth International Congress of Refrigeration," Venice, Italy, Sept. 1979 (In press).

[6] Zimmerman, J. E. and Sullivan, D. B., Cyogenics 19, pp. 170-171, (1979).

[7] Zimmerman, J. E. in "Future Trends in Superconductive Electronics," (B. S. Deaver, Jr., C. M. Falco, J. Harris, and S. W. Wolf, eds.) AIP Conf. Proceedings Number 44, New York, American Institute of Physics, 1978, pps. 412-420.

[8] Sullivan, D. B. and Zimmerman, J. E., Int. J. of Refrig. 2, pps. 211-213, (1979).

[9] Zimmerman, J. E. in "Proceedings of the SQUID Geophysics Workshop," Los Alamos, NM, June 1980. (In press).

[10] Zimmerman, J. E. in "SQUID 80: Superconducting Quantum Interference Devices and their Applications" (H. D. Hahlbohm and H. Lubbig, eds.) Berlin, Walter de Gruyter (1980), pps. 424-443.

[11] Sullivan, D. B. , Zimmerman, J. E., and Ives, J. T. in "Refrigeration for Cryogenic Sensors and Electronic Systems," NBS Special Publication SP-607, (J. E. Zimmerman, D. B. Sullivan, and S. E. McCarthy, eds.), Superintendent of Documents, U. S Government Printing Office, Washington, D. C. 20234 (1981). (Also available from the authors), pps. 186-194. 
[12] Zimmerman, J. E., Sullivan, D. B., Kautz, R. L., and Hobbs, R. D., See ref. 11, pps. 173-177.

[13] Cox, J. E. and Wolf, S. A., see ref. 3, pps. 123-129.

[14] Nisenoff, M., see ref. 11, pps. 195-209. 
7. Appendix - Publications generated by the Cryocooler Program. The original page numbers have been deleted so as to provide continuous numbering in this volume. Original page numbers are included in the reference at the beginning top of each publication.

1. Possible Cryocooler for SQUID Magnetometers . . . . . . . . . . . . . . . . $\frac{\text { Page }}{20}$

2. Refrigeration for Small Superconducting Devices . . . . . . . . . . . . . . 30

3. Operation of a SQUID in a Very Low-Power Cryocooler . . . . . . . . . . . . . . . . . . 38

4. Shuttle Heat Transfer in Plastic Displacers at Low Speeds . . . . . . . . . . . . . . 45

5. Analysis of Regenerator Inefficiency for Stirling-Cycle Refrigerators with Plastic Displacers . . . . . . . . . . . . . . . . . . . . . . 52

6. A Milliwatt Stirling Cryocooler for Temperature below 4 K . . . . . . . . . . . 58

7. Cryocoolers for Superconductive Electronics . . . . . . . . . . . . . . . 60

8. Very Low-Power Stirling Cryocoolers Using Plastic and Composite Materials . . . . . . . 69

9. Cryocoolers for Geophysical SQUID Magnetometers . . . . . . . . . . . . . . . . 72

10. Cryogenics for SQUIDs . . . . . . . . . . . . . . . . . . . . . . 75

11. Operation of a Practical SQUID Gradiometer in a Low-Power Stirling Cryocooler . . . . . 96

12. Measurement of Thermal Properties of Cryocooler Materials . . . . . . . . . . 105 
"Superconducting Quantum Interference Devices and their Applications," (H. D. Hahlbohm and H. Lübbig, eds.) Berlin, Walter de Gruyter (1977), pps. 287-296.

POSSIBLE CRYOCOOLERS FOR SQUID MAGNETOMETERS*

J. E. Zimmerman, R. Radebaugh and J. D. Siegwarth Cryogenics Division, National Bureau of Standards, Boulder, Colorado 80302 USA.

\section{ABSTRACT}

In a study to determine if a cyclic cryogenic refrigerator is suitable for cooling SQUID magnetometers and similar instruments, we have used a specially designed stirling machine with a three-stage displacer to achieve and maintain a temperature of $13 \mathrm{~K}$. The displacer and cylinder were made of low-susceptibility, nonconducting plastic to minimize magnetic interference. For the same reason, and also to minimize mechanical noise, the machine operates at low displacement and low speed, and it uses only about $10 \mathrm{~W}$ of mechanical power, requiring $50 \mathrm{~W}$ input to a typical small electric motor. Since the temperature achieved is within the range of $\mathrm{NbN}$ and $\mathrm{Nb}_{3} \mathrm{Sn}$ SQUIDs, it can,

in principle, be used to cool a SQUID. The estimated magnetic signal from the reciprocating displacer may not seriously affect the magnetometer sensitivity, but the signal due to the cyclic pressure-induced geometric distortion of the SQUID mounting in the earth's field may be very difficult to eliminate.

\section{INTRODUCTION}

In this paper we address a subject that is always implicit, but not usually expressed in detail, in any application of superconductivity, namely, the cryostat. For small devices, such as SQUIDs, the universal practice is to use liquidhelium cryostats with a variety of shapes, sizes, and special features. (1) It need hardly be pointed out that the cost and inconvenience of procuring and handling liquid helium, the attendant restrictions on how the instrument can be mounted and handled, and the inherent intermittent nature of the operation, all contribute to the obvious disadvantage of superconducting devices relative to competing devices operating at or near ambient temperature.

The alternative to liquid-helium cooling is to use a closedcycle cryogenic refrigerator (hereafter referred to as a cryocooler). The use of a cryocooler replaces the disadvantageous features noted above with a whole set of new ones. Limited reliability is perhaps the most often mentioned; others are maintenance requirements, cost, and, as we shall see, magnetic and mechanical interference due to moving parts. with regard to reliability, there seem to be two schools of thought, corresponding roughly with the high reliability expounded by cryocooler manufacturers on the one hand, and the

${ }^{\star}$ Contribution of NBS, not subject to copyright. 
low reliability expected by potential users on the other. (2) The fact is that very few cryocoolers are in use today to operate small superconducting devices, so there is no substantial body of experience regarding reliability or anything else.

There are several intermediate solutions to the cryogenic cooling problem. One of these is a liquid-helium cryostat incorporating one or more radiation shields maintained at rather low temperatures by an auxiliary cryocooler, which may greatly reduce the helium evaporation rate. (3) Such a system combines some of the best, and some of the worst, features of both worlds. One of the good features is that it permits one to get practical experience in the use of cryocoolers without being fully committed, in the sense that if the cryocooler turns out to be no good one still has a cryostat. Another useful feature is that the cryocooler can be turned off for periods of several hours of critical measurements, where the magnetic and mechanical noise of the cryocooler might be intolerable, with only a slight increase in liquid-helium consumption.

There are some other types of cryostats which may be useful for particular applications. For example, a cascaded JouleThomson system operating from cylinders of compressed gases may be suitable for intermittent short-term operation with quick cooldown. . It is beyond the scope of this paper to discuss all possible cryostatic systems, even by way of introduction, and we will hereafter restrict the discussion to the use of liquid-helium and closed-cycle refrigerators (cryocoolers) using helium gas as the working fluid.

In fact, there is only one known type of practical, small, self-contained system which can maintain superconducting temperatures in a $300 \mathrm{~K}$ environment. This is the type using helium gas as a working fluid in a cycle involving isothermal compression at room temperature and isothermal expansion, or a combination of isothermal expansion and other processes, at one or more low temperatures. The particular point to be emphasized here is that compression and expansion require moving parts, which may be very difficult to operate in a way that will not interfere with the operation of highly sensitive magnetometers.

We have chosen the stirling machine as an analytical and experimental model to investigate the problems associated with operating SQUID magnetometers with a small closed-cycle heliumgas cryocooler. It is a very simple machine, shown schematically in Figure 1, which has only two essential moving parts, a piston and a displacer. (4) The displacer fits loosely in its cylinder so that gas can move freely past it. Thus the pressure $P$ is nearly the same throughout the total volume $V$ of 


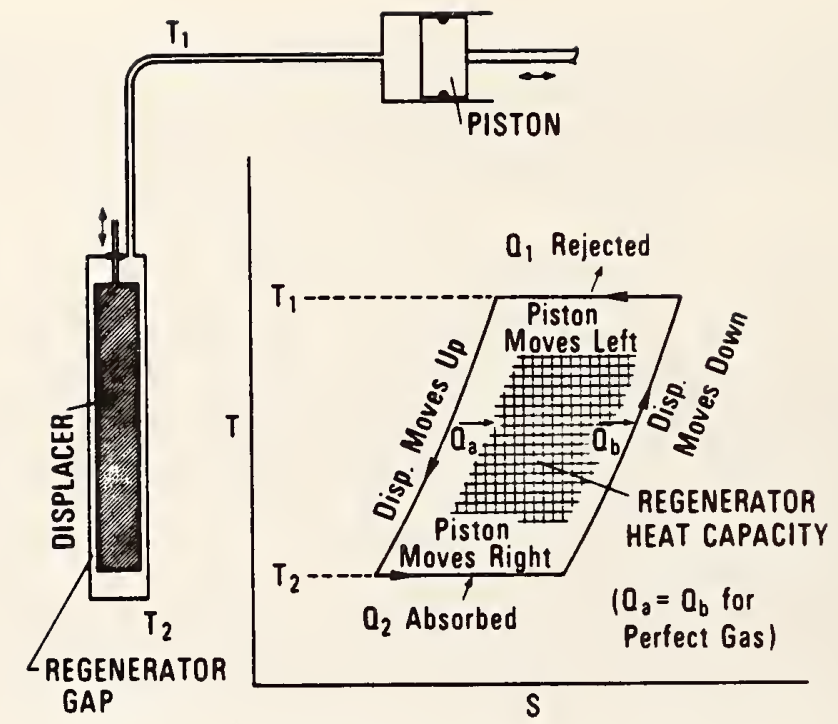

Figure 1 Schematic representation of operation of split stirling machine and idealized thermodynamic cycle on temperature-entropy diagram.

the system. Work $W=\int P d V$ may be done on the system as the piston moves back and forth, but no work is done by or on the displacer since displacer motion does not change the volume. Referring to Fig. l, when the displacer is in its lowest position, the piston is moved to the left, compressing the working fluid and producing a heat of compression $Q_{1}$ which is rejected at ambient temperature $\mathrm{T}_{1}$, typically $300 \mathrm{~K}$. Next, the displacer is moved to the top of its cyliner, so the working fluid moves (is "displaced") from the top to the bottom of the cylinder. If we may assume that the machine is already in steady-state operation, with the bottom end at a low temperature $T_{2}$, and a stable temperature gradient already established along the displacer, then the fluid being displaced gives up heat $Q_{a}$ to the displacer and cylinder walls along the annular gap, and arrives at the bottom end at temperature $T_{2}$. Then the piston is moved to the right, expanding the fluid and producing a flow of heat $Q_{2}$ into the fluid at temperature $T_{2}$ at the bottom of the cylinder. Finally, the displacer is moved to its lowest position, displacing the remainder of the fluid back to the top of the cylinder and completing the cycle. The fluid picks up heat $\mathrm{Q}_{b}$ from the walls of the annular gap as it travels up from $\mathrm{T}_{2}$ to $\mathrm{T}_{1}$.

In steady state operation it is required that $Q_{a}=Q_{b}$, otherwise the temperature gradient along the displacer and 
cylinder will change with time. With real gases, such as helium at $20 \mathrm{~K}$ and below, the requirement $Q_{a}=Q_{b}$ is incompatible with the assumption of isothermal heat exchange $Q_{1}$ and $Q_{2}$ at $T_{1}$ and $T_{2}$. (i.e., The enthalpy change $Q_{a}$ or $Q_{b}$ between two given temperatures $T_{1}$ and $T_{2}$ depends on pressure, so the requirement $Q_{a}=Q_{b}$ means that the expansion cannot be strictly isothermal. This is a limitation, or at least an analytical complication, in the operation of the stirling cycle.

A more serious limitation, which we will have more to say about, is that the heat capacity of the walls at the cold end becomes insufficient, relative to that of the fluid, to efficiently provide the necessary heat exchange $Q_{a}$ and $Q_{b}$. Considerable research has been devoted to finding materials with favorable properties (large heat capacity and thermal conductivity) to use in regenerative heat exchangers at low temperatures. For use with SQUID magnetometers we would like to impose additional restrictions on the material, namely, that it also have favorable properties relative to generation of electromagnetic interference and noise in the SQUID.

\section{EXPERIMENTS WITH PLASTIC DISPLACER-REGENERATORS}

Our experiments are intended only to demonstrate feasibility of specific materials in a specific refrigerating mechanism which may have a possibility of satisfying the stringent requirements of a SQUID magnetometer.

The first experiment was to build a singlestage displacerregenerator (Fig. 2) of the type invented by Stirling in 1816 , but using modern materials. The displacer was a nylon rod 9.6 $\mathrm{mm}$ diameter by $300 \mathrm{~mm}$ long. It operated inside a spun-glass epoxy cylinder (designated G-10 by the National Electronic Manufacturers Association) with $2.4 \mathrm{~mm}$ wall thickness. The radial clearance between displacer and cylinder was about .07 $.07 \mathrm{~mm}$, and the length of the cylinder was $313 \mathrm{~mm}$, allowing a $12.7 \mathrm{~mm}$ stroke for the displacer, which was connected to a thin brass push rod running through a sliding rubber seal at one end of the cylinder. The push rod was operated by a scotch yoke at the end of a shaft which was turned at $1 \mathrm{~Hz}$ by a small electric motor. A pair of cams, $90^{\circ}$ out of phase, on the same shaft, operated the inlet and exhaust valves.(5) The working fluid was helium gas, supplied at 14 atmospheres from a storage cylinder and exhausted to the atmosphere when expanded. Twenty-five layers of aluminized plastic sheet were wrapped around the displacer cylinder, which was mounted in an evacuated metal cylinder. Operated at $1 \mathrm{~Hz}$, the machine attained a temperature in the neighborhood of $50 \mathrm{~K}$ three or four hours after starting, and maintained the temperature for about two days on a cylinder of helium. 


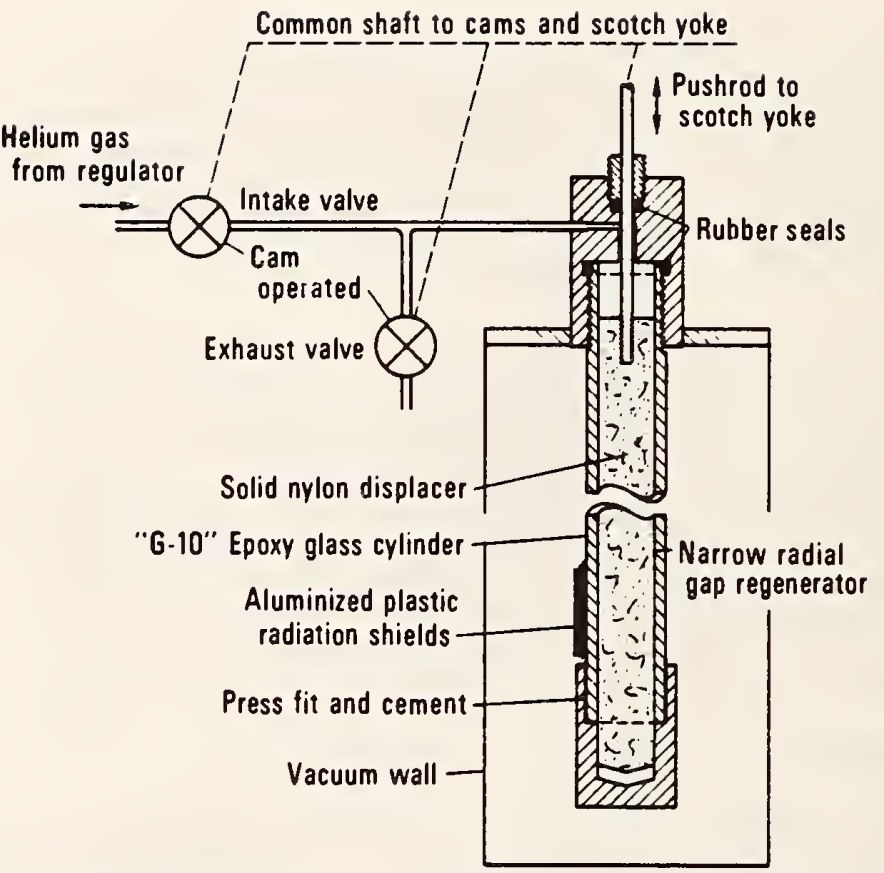

Figure 2 Details of simple cryocooler operating from compressed helium gas cylinder. Radial gap around displacer is too narrow $(20.05 \mathrm{~mm})$ to be shown in this drawing. Displacer was $9.5 \mathrm{~mm}$ diameter by $300 \mathrm{~mm}$ long, with $12.7 \mathrm{~mm}$ stroke.

The success of this simple apparatus in producing rather low temperatures encouraged us to construct a 3-stage, closedcycle, split Stirling machine, (6) using the same materials and techniques (Fig. 3). Dimensions of the stages were as follows: The first stage displacer-regenerator was $245 \mathrm{~mm}$ long and $19 \mathrm{~mm}$ diameter, with $0.05 \mathrm{~mm}$ radial clearance inside the spun-glass epoxy cylinder. The second stage was $143 \mathrm{~mm}$ long and $9.45 \mathrm{~mm}$ diameter with $0.07 \mathrm{~mm}$ radial clearance. The third stage was $144 \mathrm{~mm}$ long and $4.7 \mathrm{~mm}$ diameter with $0.04 \mathrm{~mm}$ radial clearance. The cylinders all had a $2.4 \mathrm{~mm}$ wall thickness and were epoxied together using a commonly available epoxy glue. A brass cap was epoxied on the bottom or cold end. Cylindrical aluminum radiation shields were fitted over the lower end of the first and second stages. Several layers of aluminized plastic sheet were wrapped within the shields and around the outside of the assembly for additional radiation shielding. A polished stainless steel cylinder containing a $35.6 \mathrm{~mm}$ diameter plastic piston formed the compressor. The various dimensions of these machines were dictated by intuition and by immediate availability of materials. In subsequent work we hope to optimize the size and shape on a more rational basis. The piston was driven through a $38 \mathrm{~mm}$ stroke by a 


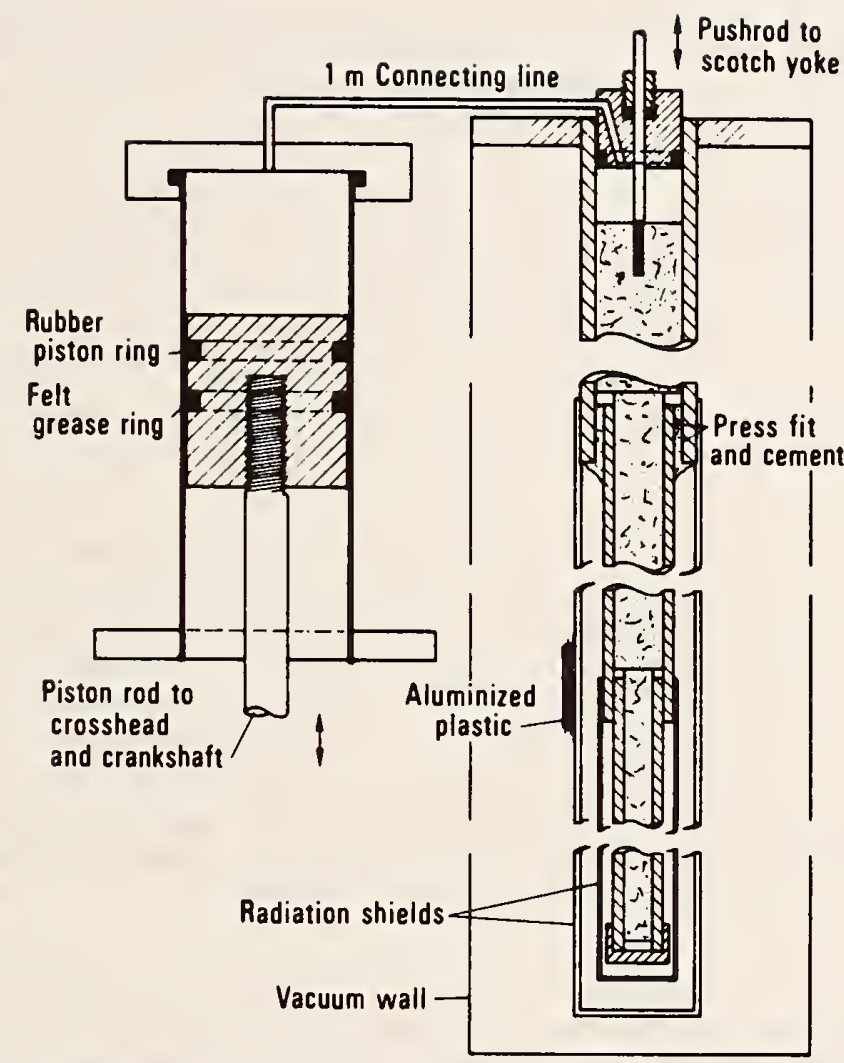

Figure 3 Three-stage Stirling cryocooler. Displacer sections were $19 \mathrm{~mm}, 9.5 \mathrm{~mm}$, and $4.7 \mathrm{~mm}$ diameter, and $245 \mathrm{~mm}$, $143 \mathrm{~mm}$, and $144 \mathrm{~mm}$ long, with $12.7 \mathrm{~mm}$ stroke. Piston was $35.6 \mathrm{~mm}$ diameter with $38 \mathrm{~mm}$ stroke.

crankshaft and crosshead mechanism adapted from a small commercial freon compressor. Operating at $1 \mathrm{~Hz}$ with a displacer stroke of $12.7 \mathrm{~mm}$ and a pressure excursion of about 3 to $13 \mathrm{~Pa}$, this machine maintained a temperature below $16 \mathrm{~K}$.

This machine was also operated with the same valved cycle used for the first cryocooler, and reached almost the same minimum temperature of $16 \mathrm{~K}$. The performance was improved by reducing the dead space around the displacer. We found that the nylon displacer had a larger coefficient of expansion than the spunglass epoxy cylinder, so that there were several millimeters of "dead space" at the cold end after steady-state operation was achieved. We therefore carefully measured the differential contraction and machined the displacer accordingly, so that the displacer and cylinder fitted together precisely in steadystate operation, rather than at room temperature. After 
initial cooldown, the dead space was eliminated by readjusting the length of the push rod. When this was done, and the phase angle between piston and displacer was empirically optimized, the cryocooler maintained a temperature around $13 \mathrm{~K}$ or less, for continuous periods as long as 5 weeks. The limit on nonstop operation appeared to be due to air being entrapped by grease on the piston ring and the pushrod seal and contaminating the helium gas. Such contamination would eventually build up, reduce the radial clearance of the displacer, and cause friction. We eliminated most of the contamination by maintaining an ambient helium atmosphere on the back side of the piston, but have not yet done so for the pushrod seal.

This three-stage displacer has been operated a total of nearly 5000 hours with no obvious signs of wear. It will maintain a temperature between $12-1 / 2$ and $13 \mathrm{~K}$ at the cold end with no heat load. With a heat load of $11 \mathrm{~mW}$ the temperature rises to $14.5 \mathrm{~K}$. The outer radiation shield is maintained at $120 \mathrm{~K}$ and the inner radiation shield at $40 \mathrm{~K}$. Operated at $\mathrm{f}=1 \mathrm{~Hz}$ with a pressure excursion as noted above, the mechanical power required for the compression stroke, assuming an isothermal process, is $P_{1} V_{1} f\left(\ln P_{2} / P_{1}\right)=\left(3 \times 10^{5}\right)\left(38 \times 10^{-\frac{1}{6}}\right) \ln (13 / 3)$ watts $\cong 15 \mathrm{~W}$, where $\mathrm{v}_{1}=38 \mathrm{~cm}^{3}$ is the piston displacement. since a considerable fraction of the work is returned on the expansion stroke, the net power required was estimated to be of the order $10 \mathrm{~W}$ or less. This estimate was confirmed by operating a similar machine with a 50 watt motor whose efficiency was less than $25 \%$.

We have recently made fairly extensive measurements and calculations of the various components of intrinsic heat load in this machine, namely, shuttle heat, conduction, radiation, and regenerator inefficiency, for the purpose of optimizing the design. In this connection, we may wish to complicate the design slightly by incorporating a high-heat-capacity matrix at the cold end of the displacer cylinder, to improve the regenerative heat exchange and achieve lower temperature. The results of our measurements and the optimization will be submitted for publication subsequently.

\section{CONSIDERATION OF COMPATIBILITY WITH A SQUID MAGNETOMETER}

Having demonstrated that superconducting temperatures (high$T_{c}$ ) can be achieved and maintained for long times with a very low-power cryocooler made of plastics with favorable magnetic properties, it remains to be seen whether such a machine can be used with a highly sensitive SQUID magnetometer. Ignoring for the present the problem of the compressor and its drive mechanism, consider the magnetic signals generated by just those parts that would be near the SQUID, namely the bottom end of the displacer and its cylinder. In our experimental 
machine, the end of the displacer is $4.7 \mathrm{~mm}$ diameter and moves up and down about $13 \mathrm{~mm}$. In the field of the earth, $0.5 \mathrm{x}$ $10^{-4} \mathrm{~T}$, this gives a peak-to-peak magnetic moment at $1 \mathrm{~Hz}$ of about $0.3 \mathrm{~cm}^{3} \times 0.5 \times 10^{-4} \mathrm{~T} \times 1.1 \times 10^{-5}=0.16 \times 10^{-9} \mathrm{~T} \mathrm{~cm}^{3}$. The susceptibility of nylon, $1.1 \times 10^{-5}$, was measured for us by F. Fickett.

If a SQUID were mounted $5 \mathrm{~cm}$ (for example) from the end of the displacer, the peak-to-peak field at $\mathrm{l} \mathrm{Hz}$ would be about $10^{-12} \mathrm{~T}$. A coherent oscillation of this magnitude could be subtracted from the output with an accuracy of perhaps $10^{-14} \mathrm{~T}$, which is comparable to present SQUID sensitivities in a $1 \mathrm{~Hz}$ bandwidth. Thus, one may be able to cope with the magnetic signal from a small reciprocating displacer of nylon or comparable material.

on the other hand, a peak-to-peak pressure excursion of $10^{6} \mathrm{~Pa}$ $\left(10^{5} \mathrm{~Pa} \cong 1\right.$ atmosphere) in the displacer cylinder will cause it to bend owing to non-uniform wall thickness and other defects. If the SQUID were mounted on the end of the cylinder for mechanical support, bending of the cylinder in the earth's field may cause a flux change in the SQUID far too large to be subtracted from the output with sufficient accuracy. There is no need to use precise numbers to show this. An elastic modulus of $10^{11} \mathrm{~Pa}$ and a pressure change of $10^{6} \mathrm{~Pa}$ in a cylinder whose ratio of length to diameter is 10 gives a bend of the order of $10 \times 10^{6} \times 10^{-11} \times 10^{-2}=10^{-6}$ radians, if the lateral inhomogeniety (different wall thickness, for example) is of order $10^{-2}$. In the earth field this would appear as an oscillation of the order of 10-10 T. Two alternative approaches suggest themselves for reducing this signal. One is to measure the bending and empirically correct it by grinding or scraping one side of the cylinder. The other is to mount the SQUID on an independent support with only a flexible thermal connection to the cold end of the cylinder. In any case, it may be quite difficult to reduce or compensate the bending signal to a level of $10^{-14} \mathrm{~T}$.

\section{COMPARISON OF THE CRYOCOOLER WITH CONVENTIONAL LIQUID-HELIUM CRYOSTATS}

We have shown that the lowpower cryocooler can maintain temperatures below $15 \mathrm{~K}$ with ten or twenty milliwatts heat load. Bias power requirements for SQUIDs are many orders of magnitude smaller than this, and the only significant heat load imposed by the SQUID is conduction down the electrical leads. our experience with conventional liquid-helium cryostats indicates that, with good design, the conduction heat load can be reduced to less than ten milliwatts. Actually, designing or specifying cryostats on the basis of refrigeration capacity at the coldest point is quite misleading. What is important 
is to distribute the refrigeration capacity, using a multistage machine, so that the bulk of the heat input (conduction, radiation, etc.) is pumped at relatively high temperatures, leaving very little to be pumped at the lowest temperature. The distribution of refrigeration capacity vs temperature is another aspect of design optimization which has received little attention in the literature. For comparison, a typical small liquid-helium cryostat with an evaporation rate of one liquid liter per day (corresponding to about 700 liters of gas at room temperature) is supporting a total heat load, intrinsic plus imposed, of $29 \mathrm{~mW}$ at $4.2 \mathrm{~K}$, and has the capability of absorbing an additional $7.6 \mathrm{~m} \mathrm{~W} / \mathrm{K}$ due to the heat capacity of the vapor, which is approxmately independent of temperature. These figures might be used as a guide for the necessary refrigeration capacity of a small cryocooler. Actually, the figures should be quite conservative because (1) a cryocooler would not have the large cold reservoir and (2) optimum design would probably not require the temperatureindependent heat absorption capacity, but rather would concentrate the refrigeration capacity at higher temperatures.

\section{FURTHER PERTINENT COMMENTS}

The work we have reported here is unique in two respects, first in the choice of materials and construction methods for the displacer, and second in building a machine with at least an order of magnitude lower input power requirements than previous comparable machines. Both of these features are, of course, related to our particular aim of minimizing magnetic interference, but they will have considerable effect on cost and portability as well. It appears from our experiments that the cost of a cryocooler could easily become comparable to the cost of a SQUID magnetometer or similar superconducting instrument. Such systems might well be justified economically, in view of the fact that the cost of liquid helium will increase as the supply diminishes, whereas the cost of cryocoolers should decrease substantially if they are produced in sufficient numbers to replace present liquid-helium cryostats.

This work completes what might be called a preliminary study of the feasibility of using a small mechanical cryocooler for SQUID magnetometers and other small superconducting devices. We conclude that a second phase of development is justified. We believe this should include (1) optimizing the design as noted above, (2) incorporating a high-T $T_{C}$ SQUID with which to measure and evaluate the level of magnetic interference from the various moving parts, and (3) evaluating other potentially useful materials and refrigeration processes which may improve the performance. 


\section{ACKNOWLEDGEMENT}

We are grateful to several individuals for helpful comments and criticism, particularly Sam Collins, Dave Daney, T. R. Strobridge, and $R$. O. Voth. This work is supported jointly by the National Bureau of Standards, the Office of Naval Resarch and the Naval Electronics Systems Command.

\section{REFERENCES}

1. For example, we have built 1.7-liter spun-glass-epoxy cryostats with hold times of nearly three days on one filling of liquid helium. At the opposite extreme, a 180-liter cryostat has been built with a hold time of 102 days. See R. J. Dinger, J. R. Davis and M. Nisenoff, NRL Memorandum Report 3256, Naval Research Laboratory, Washington, DC (March, 1976).

2. Some contrasting comments on reliability by different people (not directed specifically at each other) may be found in Proc. Cryogenic Cooler Conf., AFFDL-TR73-149, Air Force Flight Dynamics Laboratory, Wright-Patterson Air Force Base, Ohio 45433 (Dec. 1973). This report also contains several papers on recent developments in miniature cryocoolers for the 10-20 $\mathrm{K}$ range.

3. R. C. Longsworth, P. 390, Proc. Fifth International Cryogenic Engineering Conf., IPC Science and Technology Press (1974), has shown that liquid-helium hold times can be increased by a factor of 4 or more by maintaining a shield at $20 \mathrm{~K}$ rather than at $77 \mathrm{~K}$. He estimates that one might achieve hold times of the order of a year with a 25-liter cryostat. His results should be quite conservative as applied to cryostats for lightweight low-energy devices like SQUIDs.

4. Much historical and recent work on stirling and related machines is described by G. Walker, stirlingCycle Machines. Oxford University Press (1973).

5. Operation of a Stirling-type displacer-regenerator with a valved helium supply was developed by $H$. 0 . McMahon and W. E. Gifford, p. 354, Advances in Cryogenic Engineering, Vol. 5, Plenum Press (1960).

6. The stepped-displacer idea used here was invented by Fokker and Köhler. The equivalance of a steppeddisplacer machine to a multi-stage machine is explained in some detail by G. Prast, Philips Tech. Rev. 26, 1 (1965). The term "split" refers to the fact that the compressor cylinder and displacer cylinder are geometrically separated from each other. 
Conference of the German Refrigeration Society, Munich (October 1976).

REFRIGERATION FOR SMALI SUPERCONDUCTING DEVICES*

J. E. Zimmerman, R. Radebaugh and J. D. Siegwarth

Cryogenics Division

National Bureau of Standards

Boulder, Colorado 80302

\begin{abstract}
The present state of the art is such that the greatest cost in using small superconducting devices, in many applications, is the expense and inconvenience of the associated cryogenic system. The practicality of such devices would be greatly enhanced, therefore, if a practical and economical self-contained, closed-cycle cryocooler could be made compatible with such devices. We have built an experimental cryocooler whose unique features (non-magnetic materials and very low input power) may represent a partial solution to the problem.
\end{abstract}

Small superconducting devices are currently operated almost universally in liquid-helium cryostats. Evaporation rates vary from less than a liter per day to several liters per day, depending upon the size and design of the cryostat and the heat load imposed upon it. Continuous operating times as long as 102 days have been achieved with a 180-liter cryostat built for a SQUID magnetometer ${ }^{1}$, which may be taken as the prototype for practical superconducting devices. Because of the increasing number of applications of such devices, we have begun a study of the possibility of maintaining the required operating temperatures with a low-power closed-cycle cryogenic refrigerator, which may have advantages of portability, versatility, and long continuous operating time. since the cost of helium will increase as the supply diminishes, and the cost of closed-cycle refrigerators should decrease substantially if they are produced in sufficient numbers to replace present liquid-helium cryostats, the shift of technology can be economically justified.

In the first part of this paper we emphasize that a need exists for a low-power, closed-cycle cryogenic refrigerator (cryocooler) for the

* Contribution of the National Bureau of Standards, not subject to copywrite. 
convenient operation of a number of small superconducting devices, which are now or may soon be used in a broad spectrum of applications, and in the second part we describe briefly our own preliminary experiments directed toward satisfying these needs.

Several superconducting devices with uniquely useful properties and an impressive range of potential applications have appeared in the last few years. A bolometer, operating at the superconducting transition temperature, is an order of magnitude more sensitive (noise-equivalent power $2 \times 10^{-15} \mathrm{w} / \sqrt{\mathrm{Hz}}$, than any other device of its type ${ }^{2}$. Similarly, a so-called super-Schottky diode, a superconducting $\mathrm{Pb}$ contact on GaAs, exhibits an $\mathrm{X}$-band noise temperature less than $10 \mathrm{~K}$, a hundred times better than conventional room-temperature diodes ${ }^{3}$. The list of applications of the Josephson effect is too long to be summarized here, and we will only mention two. A microwave-biased Josephson junction has replaced the electrochemical cell as the primary voltage standard in the leading standards laboratories of the world. Superconducting quantum interference devices (SQUID's) in many sizes and shapes are used in hundreds of laboratories as magnetometers, galvanometers, susceptibility meters, dilatometers, rf power meters and rf attenuation standards, vlf communication receivers, and many other purposes ${ }^{4}$.

In terms of sheer number of uses and users, the SQUID magnetometer is clearly the instrument with greatest practical impact at the present time, compared to other superconducting devices. In addition to numerous applications in low-temperature physics laboratories, it is being used by significant numbers of specialists in entirely different fields, e.g., geology ${ }^{5}$, medical research ${ }^{6}$, and military purposes ${ }^{7}$. In all such applications, the users accept the inconvenience and cost of a cryogenic system because a SQUID magnetometer is a simple and rather inexpensive instrument, and it is more sensitive, e.g., $10^{-14} \mathrm{~T} / \sqrt{\mathrm{Hz}}$, than any comparable conventional instrument by several orders of magnitude.

All or most of the devices listed above are so superior, in sensitivity and other important respects, to conventional room-temperature devices, that there is little doubt they would predominate in practically all applications, were it not for the associated cryogenic system. 
Both the first cost and the operating cost of present cryogenic systems usually far exceed the cost of a device like a SQUID or a superconducting bolometer. Thus, it is the cryogenic systern which currently limits the acceptance of these devices, not the characteristics of the devices themselves. Viewed in this light, it is seen that an order of magnitude improvement in the cryogenic system (involving such qualities as cost, operating convenience, size, reliability, etc.) might well result in an enormous enhancement of the user acceptance.

Another feature common to the devices listed above is that their intrinsic refrigeration requirements are microscopic. The bias power, essentially all of which is dissipated and must therefore be absorbed by the cryostat, varies from $10^{-10} \mathrm{w}$ for the bolometer, to $10^{-8} \mathrm{~W}$ for the diode, to $10^{-12} \mathrm{~W}$ for an $\mathrm{rf}-\mathrm{biased}$ SQUID. Room-temperature radiation to surfaces with an absorptivity of say 28 would amount to about $10^{-3} \mathrm{~W}$, assuming that the cold device has a surface area of the order of $1 \mathrm{~cm}^{2}$. It is difficult to make any general estimates of heat conduction along the mechanical supports and electrical leads, since design requirements might be quite varied.

An empirical upper limit of refrigeration required is obtained from our experience with the operation of SQUID magnetometers in liquidhelium cryostats. We have observed a difference of evaporation rate of the order of $0.1 \mathrm{l} /$ day with and without the SQUID in the cryostat, corresponding to an extra heat input into the liquid helium of $3.5 \mathrm{mw}$ due to the SQUID. It is not our purpose here to develop careful theoretical estimates of the intrinsic refrigeration requirements for small superconducting devices. However, very rough estimates show in principle (not necessarily implying practicability) that an optimum system should require several orders of magnitude lower refrigeration power than any existing practical cryocooler is capable of providing. On the basis of all the experimental and analytical evidence, we felt it would be useful to experiment with the design of a very-low-power closedcycle cryocooler, simply disregarding the small heat load imposed by the superconducting device. Our multiple uses of a SQUID magnetometer led us also to consider the possibility that the cryocooler could have a sufficiently low level of magnetic interference as not to seriously degrade 
the sensitivity of this particular instrument. Ideally, this means that the interference level should not exceed about $10^{-14} \mathrm{~T}$, although in specific applications a greater level might be tolerable. For niobium point-contact SQUID's in use at the present time the required operating temperature is about $9 \mathrm{~K}$ or below. Thin-film SQUID's of high-T alloys have been demonstrated to operate as high as $16 \mathrm{~K}^{(8)}$.

We have built a laboratory model cryocooler, with the short-term objective of mounting in it a magnetoneter SQUID and experimentally measuring the level of magnetic interference. The ultimate aim is to produce a practical self-contained cryocooler-magnetometer system. The present model is a split stirling machine (see Figure 1), with piston and displacer in separate cylinders connected by $1 \mathrm{~m}$ of $2 \mathrm{~mm}$ inside diameter tubing. Swept volume of the piston is $38 \mathrm{~cm}^{3}$, swept volume of the displacer is $3.6 \mathrm{~cm}^{3}$, and the displacer was made of solid nylon rods with three cylindrical sections $19 \mathrm{~mm}, 9.5 \mathrm{~mm}$, and $4.7 \mathrm{~mm}$ in diameter (see Figures 2 and 3 ). Thus, during the expansion stroke of the piston, refrigeration is produced at the two steps as well as at the small end, the expansion volumes being $2.7 \mathrm{~cm}^{3}, 0.68 \mathrm{~cm}^{3}$ and $0.23 \mathrm{~cm}^{3}$ respectively, totaling $3.6 \mathrm{~cm}^{3} .(9)$ The displacer cylinder was assembled from three pieces of commercially available glass-reinforced epoxy tubing. The displacer was carefully machined to fit the cylinder with less than $0.1 \mathrm{~mm}$ radial gap on all three sections. Regenerative heat exchange takes place between the working fluid (helium) and the solid surfaces along this gap, exactly as in Stirling's original engine of 1816. (10)

Thin tubular aluminum radiation shields were pressed onto the two steps on the cylinder. The piston and the displacer were coupled by appropriate mechanisms to a common crankshaft, arranged so that the phase relation between the two could be empirically adjusted for optimum results.

This machine has been operated a total of about 5000 hours at 1 stroke per second with the helium pressure oscillating between 3 and $12 \times 10^{5} \mathrm{~N} / \mathrm{m}^{2}$. After some minor initial alterations it maintained a temperature at the small end of the displacer cylinder of $13 \mathrm{~K}$, well within the operating requirement of a high-T sQuID. It required $50 \mathrm{w}$ input to an electric drive motor whose measured efficiency was less than 
258. Thus, the actual mechanical power input was approximately $10 \mathrm{w}$.

This agrees reasonably well with the power calculated from the piston displacement and the pressure excursion (1l).

We wish to emphasize that our cryocooler design represents only a tentative first step toward a possible practical machine for small superconducting devices, the realization of which seems to us potentially very rewarding. We have demonstrated ( 1 ) that non-magnetic, insulating materials such as nylon and glass-reinforced epoxy can be used in reciprocating machines with rather long operating time, and (2) that useful cryogenic temperatures can be maintained with very low input power ${ }^{(12)}$, if the superconducting system is compatible with very low refrigeration capacity.

This work is supported in part by the office of Naval Research and by the Naval Electronics Systems Laboratory. 
1. J. R. Davis and M. Nisenoff, International Conference on Superconducting Quantum Devices, Berlin (West), October 5-8, 1976. (Proceedings to be published by Walter de Gruyter, Berlin).

2. J. Clarke, Proceedings of the Berlin conference cited in ref. 1 . See also Proc. 14th Int. Conf. on Low Temperature Physics 4,226 (North-Holland Publ. Co., Amsterdam, 1975).

3. M. McColl, et al, Appl. Superconductivity Conference in Palo Alto (California), August 1976 (To be published in IEEE Trans on magnetics).

4. Several of these applications were described at the Berlin conference cited in Reference 1.

5. N. V. Frederick, et al, IEEE Trans. on Geosci. Electronics GE-12, 102 (1974). J. E. Zimmerman and W. H. Campbell, Geophysics 40, 269 (1975).

6. D. Cohen, IEEE Trans on Magnetics MAG-11 694 (1975).

7. W. M. Wynn, et al, IEEE Trans. on Magnetics MAG-11, 701 (1975) (See also Reference 1).

8. T. Fujita, et al, IEEE Trans. on Magnetics MAG-11, 739 (1975).

9. The principle of Stirling machines with multiple expansion spaces is described in detail by G. Prast, Philips Tech. Rev. 26, 1 (1975).

10. See for example G. Walker, Stirling-Cycle Machines, Oxford University Press (1973).

11. More details on the operation of this cryocooler and the possibility of operating a SQUID magnetometer with it were given at the Berlin conference cited in Reference 1 .

12. The lowest input power requirement for any previous practical cryocooler has been given as about $300 \mathrm{~W}$. See a survey by T. R. Strobridge, NBS Tech. Note 655 (June 1974). 


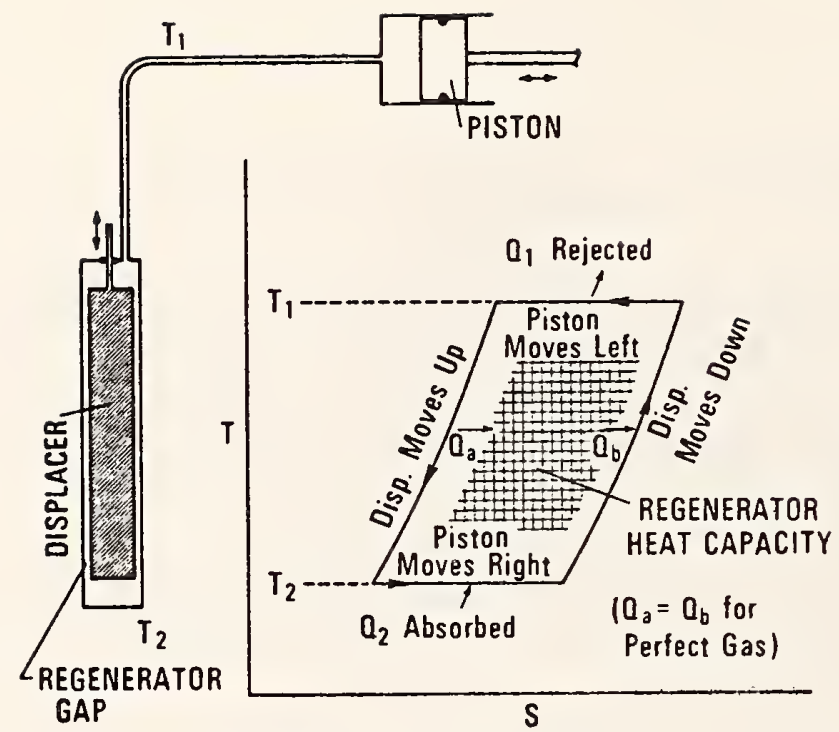

Figure 1. The essential elements of a stirling machine, and the theoretical thermodynamic cycle (from ref. 11).

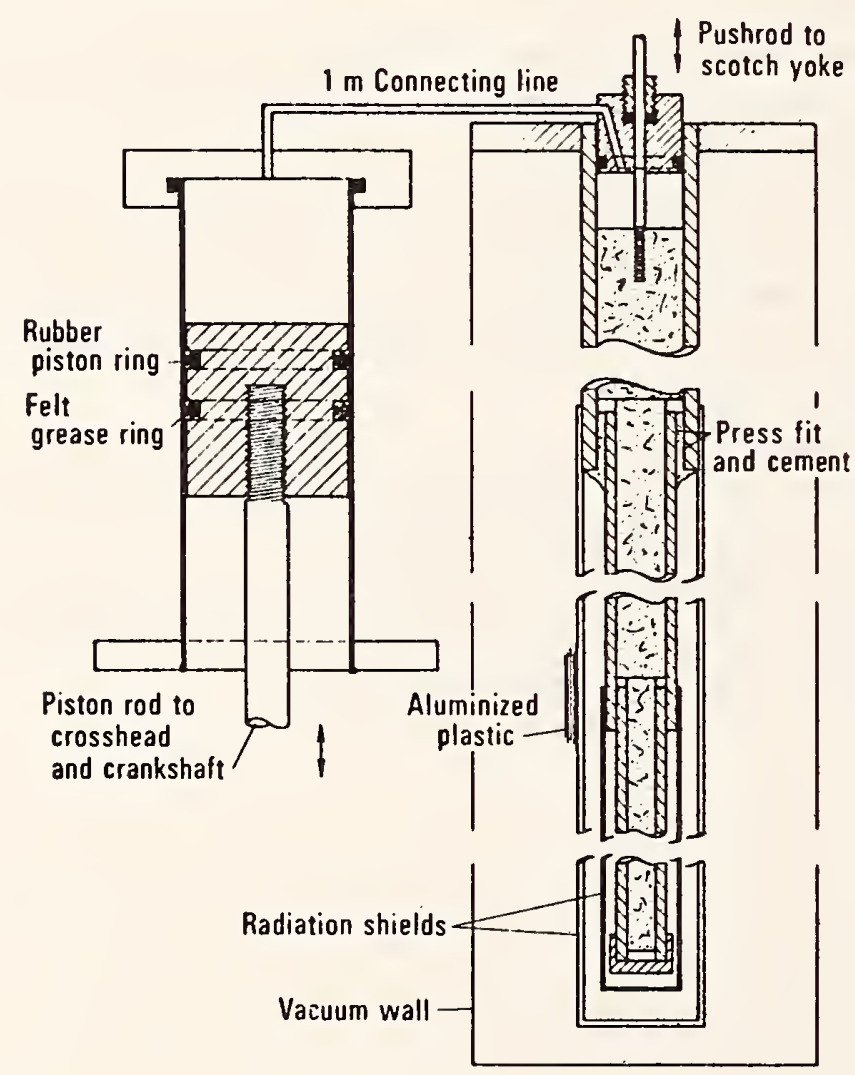

Figure 2. Sketch of an experimental three-stage Stirling cryocooler (from ref. 11). 


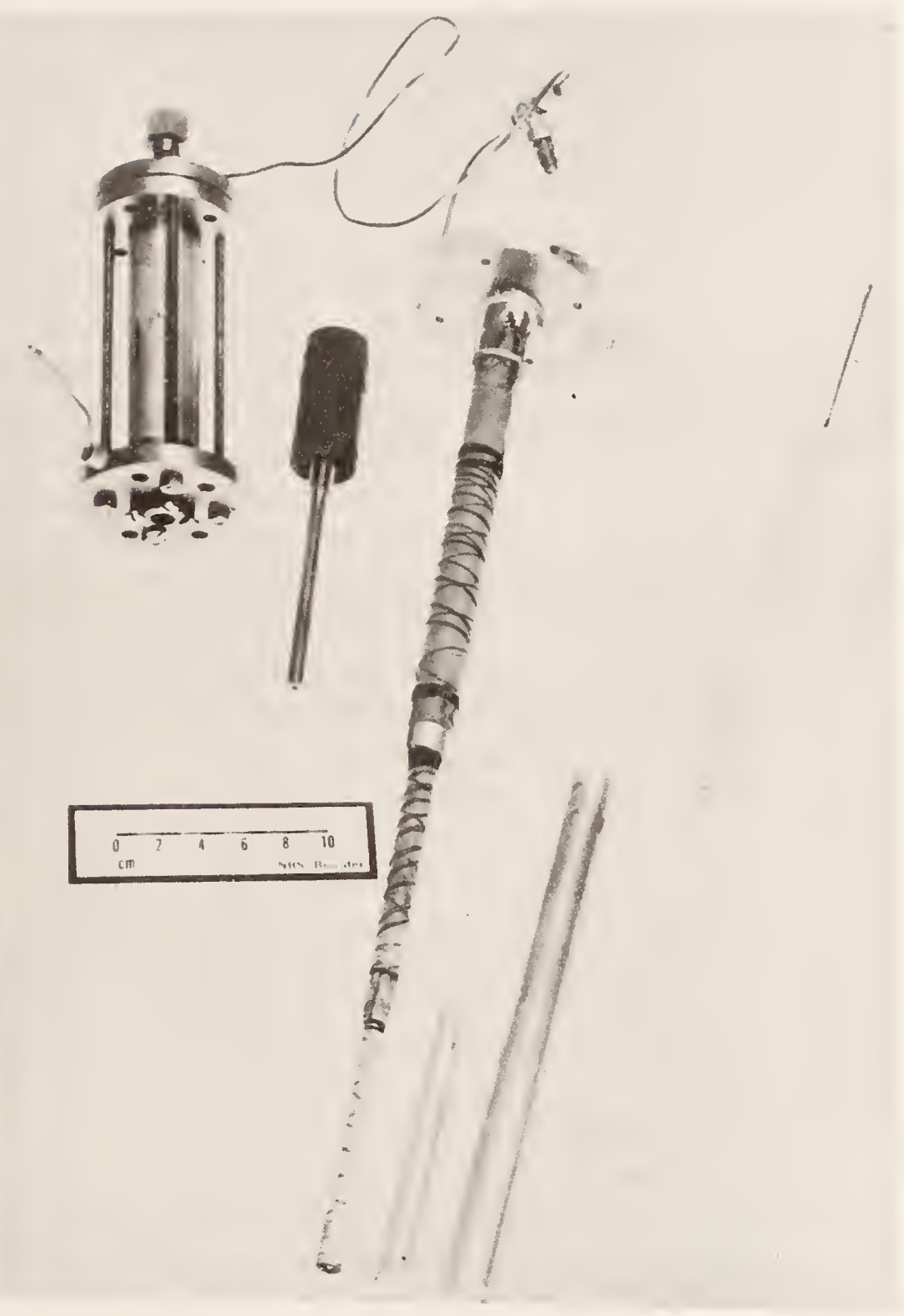

Figure 3. Photograph of disassembled cryocooler. Left to right: working cylinder, piston, displacer cylinder, two radiation shields, and displacer. Fittings were provided for maintaining a helium atmosphere on the non-working side of the piston (tube at center left), connecting a pressure transducer (top left), and filling the system with helium, (top center). 
"Applications of Closed-Cycle Cryocoolers to Small Superconducting Devices," NBS Special Publication SP-508 (J. E. Zimmerman and T. M. Flynn, eds.), Superintendent of Documents, U. S. Government Printing Uffice, Washington, D. C. 20234 (1978), pps. 59-65.

\author{
Operation of a SQUID in a Very Low-Power Cryocooler * \\ J.E. Zimmerman and Ray Radebaugh \\ Cryogenics Division \\ National Bureau of Standards \\ Boulder, Colorado 80303
}

\begin{abstract}
A point-contact Nb SQUID has been operated a total of several hundred hours at a temperature of about $8.5 \mathrm{~K}$ in a low-power Stirling cryocooler with a fourstage displacer. The system requires the order of $15 \mathrm{~W}$ of mechanical drive power. Except for the drive motor, the entire unit is non-magnetic, and the displacer and cold cylinder are made of non-conducting plastics, to minimize ferromagnetic and eddy-current fields, which would interfere with the operation of the SQUID when used as a magnetometer. With the system operating in the earth's field $B_{e}$, an ac interfering signal of about $10^{-5} B_{e}\left(i . e ., \sim 10^{-9} \mathrm{~T}\right.$ ) at the SQUID was seen at the $\mathrm{Hz}$ operating frequency of the cryocooler. This signal was probably caused by rotation of the SQUID in the earth's field due to pressure flexing of the cold cylinder, although magnetic impurities in the moving parts would also contribute. Measurements of the complete self-generated interference spectrum, and experiments aimed at reducing interference to levels of $10^{-12} \mathrm{~T}$ and below are in progress.
\end{abstract}

We have previously published an essentially theoretical discussion of the operation of a very-low-power superconducting device, such as a SQUID, in a closed-cycle cryogenic refrigerator (cryocooler) [1,2]. It was pointed out that the refrigeration capacity required for the devices themselves is negligible, and the capacity required to cool the electrical leads is small. On the other hand, a SQUID magnetometer, in particular, places a rigorous upper limit on the allowable level of magnetic and mechanical interference.

It is assumed, of course, that all of the major heat loads (room temperature radiation, and heat flow along electrical leads and support members, for example) are intercepted and pumped out at one or more higher-temperature refrigeration points, rather than at the cold end. In fact, in most small-device applications, it would seem that the refrigeration requirement at the cold end is almost incidental, if the heat loads at the higher-temperature points are handled efficiently. Obvious though this point is, cryocoolers are very often described in terms of cold-end refrigeration capacity only.

In liquid-helium cryostats, higher-temperature refrigeration is provided by the helium vapor, whose total refrigeration capacity, uniformly distributed in temperature, is about 70 times that of the evaporating liquid itself. In a cryocooler one has the opportunity, in principle, of optimizing the distribution of refrigeration at several points between ambient and low temperature regions. Furthermore, a cryocooler is required to cool only the device

* Contribution of the National Bureau of Standards, not subject to copyright. Supported in part by the Naval Electronics System Command. 
itself and not a large liquid reservoir. Thus, for two reasons, estimates of refrigeration requirements based on experience with liquid-helium cryostats may be quite conservative.

Since there is a pervasive casual tendency to specify cryocoolers only by their refrigeration capacity at cold end, it may be useful to illustrate this point with a hypothetical, but fairly typical, example. A one-liter plastic liquid-helium cryostat with a single vaporcooled radiation shield at a steady-state temperature of $150 \mathrm{~K}$ might have a heat leak to the helium, as measured by the evaporation rate, of $30 \mathrm{~mW}$. Here the evaporation rate and the shield temperature are mutually interdependent. That is, a lower shield temperature would reduce the evaporation rate, but then the flow of cold vapor would not maintain the shield temperature at the lower value. On the other hand, if a cryocooler were used to cool the same one-liter volume and the same radiation shield, it could be designed to maintain the shield at $50 \mathrm{~K}$ instead of $150 \mathrm{~K}$. If the heat leak to the one-liter volume were primarily thermal radiation, then it would be reduced by a factor of 81 , from $30 \mathrm{~mW}$ to about $0.4 \mathrm{~mW}$. If there were appreciable thermal conduction as well as radiation, the comparison would not be quite so dramatic, but still impressive.

We also described $[1,2]$ an experimental cryocooler particularly suited for operating very low-power high-T superconducting devices. This cryocooler was a three-stage split Stirling-cycle [3] machine, which was operated more than 6000 hours at 1 stroke-per-second, and reliably maintained a temperature of $13 \mathrm{~K}$ or less for uninterrupted periods of up to 5 weeks. Its novel features were (1) non-magnetic, non-conducting plastics for the displacer and cold cylinder, (2) low operating power, and (3) mechanical simplicity. Solid nylon rod was used for the displacer and commercial spun-glass epoxy ("G-10") tubing for the cold cylinder. The input power of $50 \mathrm{~W}$ to an electric drive moter with about $15 \mathrm{~W}$ mechanical output power was a factor of 10 or more smaller than the power requirements of any previous machine for this temperature range, so far as we are aware. Regenerative heat exchange occurred in the narrow radial gap between the nylon displacer surface and the inner surface of the cold cylinder. Gap regeneration was used in Stirling's original engine in 1816, and, being mechanically trivial, is commonly used in miniature engines described in hobbyists magazines.

A machine similar to ours (plastic displacer, gap regeneration, and very low power) was built several years ago by du Pre and Daniels [4]. They achieved $130 \mathrm{~K}$ with a two-stage displacer and $98 \mathrm{~K}$ with a conical displacer. The latter may be regarded as the limiting case of a displacer with an infinite number of stages.

The temperature maintained by our three-stage machine is well within the range of high-T $c$ superconductors such as $\mathrm{NbN}$ and $\mathrm{Nb}_{3} \mathrm{Sn}$. Practical devices have been made to operate at $14 \mathrm{~K}$ and above [5].

A new four-stage displacer and cold cylinder (Figures 1,2 and 3 ) were built to replace the three-stage unit. With this modification and some minor refinements, the machine has maintained around $8.5 \mathrm{~K}$ for a total of several hundred hours of operation. The four sections of the nylon displacer were, respectively, 4.7, 9.5, 19 and $28 \mathrm{~mm}$ diameter and 15, 10, 12 and $12 \mathrm{~cm}$ long. The cold cylinder was made from sections of commercial spun-glass epoxy tubing ("G-10") with 2.4 to $4 \mathrm{~mm}$ wall thicknesses. The displacer sections were carefully machined to fit the cylinder with almost no radial clearance, except for a few $\mathrm{cm}$ of the 
large end where about $0.1 \mathrm{~mm}$ radial clearance was provided. During cooldown, differential contraction of the nylon rods and the glass-epoxy tubes provides about one percent radial clearance, except at the large (warm) end. This technique of fitting the displacer to the cylinder is quite simple, and it also gives the minimum possible regenerator gap volume, which is important for achieving minimum temperature. As in the earler model, the exact lengths of the sections were calculated to take account of differential contraction, so as to give precise longitudinal fit in each section after cooldown. Here the fitting technique is not so simple. The important thing is to avoid any appreciable dead volume at the small (cold) end. slight misfitting at the larger steps seemed to have little effect on the cold-end temperature.

Our measurements of the various components of stray heat inputs, including "shuttle heat," reported separately [6], led us to conclude that lower temperature would be achieved by reducing the displacer stroke. Thus, the four-stage displacer has been operated with a $7 \mathrm{~mm}$ stroke, as compared to the $12 \mathrm{~mm}$ stroke of the three-stage model. Other operating parameters were essentially the same for both models, namely: operating speed $1 \mathrm{~Hz}$, average pressure $0.5 \mathrm{MPa}(5 \mathrm{~atm})$, and piston displacement $38 \mathrm{~cm}^{3}$. The average pressure, the speed, and the displacer stroke could be varied considerably around these values without changing the coldend temperature very much. It was found that the values of these three parameters giving lowest ultimate temperature were generally lower than the values giving fastest cooldown -a result not entirely unexpected.

The machine was mostly operated with the cold end down. However, it was also operated for a few hours with the cold cylinder horizontal, and also with the cold end up. In the latter case, the steady-state temperature was about $0.2 \mathrm{~K}$ greater than for the other two positions, e.g., $8.7 \mathrm{~K}$ instead of $8.5 \mathrm{~K}$. We did not investigate the reason for this small gravitational effect.

Temperature of the first (large) step was measured with a thermocouple, and the cold-end temperature was measured by a helium gas bulb clamped directly to the small ( $1 \mathrm{~cm}$ diameter) end of the cold cylinder. A simple permanently-adjusted point-contact $r f$-biased Nb SQUIO was clamped to the gas bulb. Low-conductivity parallel wires were used as rf coil leads, rather than the usual copper coaxial line, in order to reduce heat leak. Temperature of the first step stabilized at values of $180 \mathrm{~K}$ for average pressure $0.5 \mathrm{MPa}$ to $150 \mathrm{~K}$ for average pressure $0.7 \mathrm{MPa}$. The lowest stable cold-end temperature was obtained at about $0.5 \mathrm{MPa}$, as noted above.

Our present data on magnetic and mechanical interference are meager. With the SQUID oriented North/South in the earth's magnetic field, there was a $0.5 \mathrm{nT}$ ac component in the output at the $1 \mathrm{~Hz}$ operating frequency of the cryocooler. This was most likely due to pressure fiexing of the cold cylinder, which would result from lateral inhomogeneities in wall thickness or elastic properties. Other lower-amplitude noise components at higher frequencies have not yet been measured or identified. The $0.5 \mathrm{nT}$ interference at $1 \mathrm{~Hz}$ is only about $10^{4}$ times greater than typical SQUID sensitivities. If the hypothesis of pressure flexing is correct, then this component could perhaps be reduced by a factor of 100 by correcting the inhomogenieties in the cylinder walls. Another factor of 100 could probably be achieved by accurately measuring the wave shape and amplitude and subtracting it from the output. In any 
case, this particular interference would not so seriously affect measurements at frequencies other than $1 \mathrm{~Hz}$.

Measurements of the full self-generated interference spectrum for the system remain to be done. At this point we believe that a useful low-cost system can be built for magnetic measurements in the picotesla range. Similar systems could be built for other device applications [2]. The Josephson voltage standard [7] seems a particularly good candidate.

Just as the point contact made Josephson devices such as SQUID's available to everyone who took the time to understand a simple but unfamiliar principle, the work reported here demonstrates that superconducting temperatures are also available to everyone who takes the time to acquire modest skill with a lathe.

\section{References}

[1] J.E. Zimmerman, R. Radebaugh and J.D. Siegwarth, "Possible Cryocoolers for SQUID Magnetometers," Superconducting Quantum Interference Devices and Their Applications. H.D. Hahlbohm and H. Lübbig, eds., Walter de Gruyter, Berlin (1977) p 287.

[2] J.E. Zimmerman, R. Radebaugh and J.D. Siegwarth, "Refrigeration for Small Superconducting Devices," Conf. German Refrigeration Society, Munich (October 1976) (in press).

[3] G. Walker, Stirling-Cycle Machines, Oxford University Press (1973).

[4] F.K. du Pré, A. Daniels, "Gap-Regeneration Method for Stirling and Similar Cycles," Progress in Refrigeration Science and Technology, Vol. 1, Proc. XIII International Congress of Refrigeration, Wash. D.C., (1971) AVI Publishing Co., Inc. Westport, Connecticut, p 137 (1973).

[5] M. Beasley in this publication.

[6] R. Radebaugh and J.E. Zimmerman, in this publication.

[7] L. Holdeman, in this publication. 


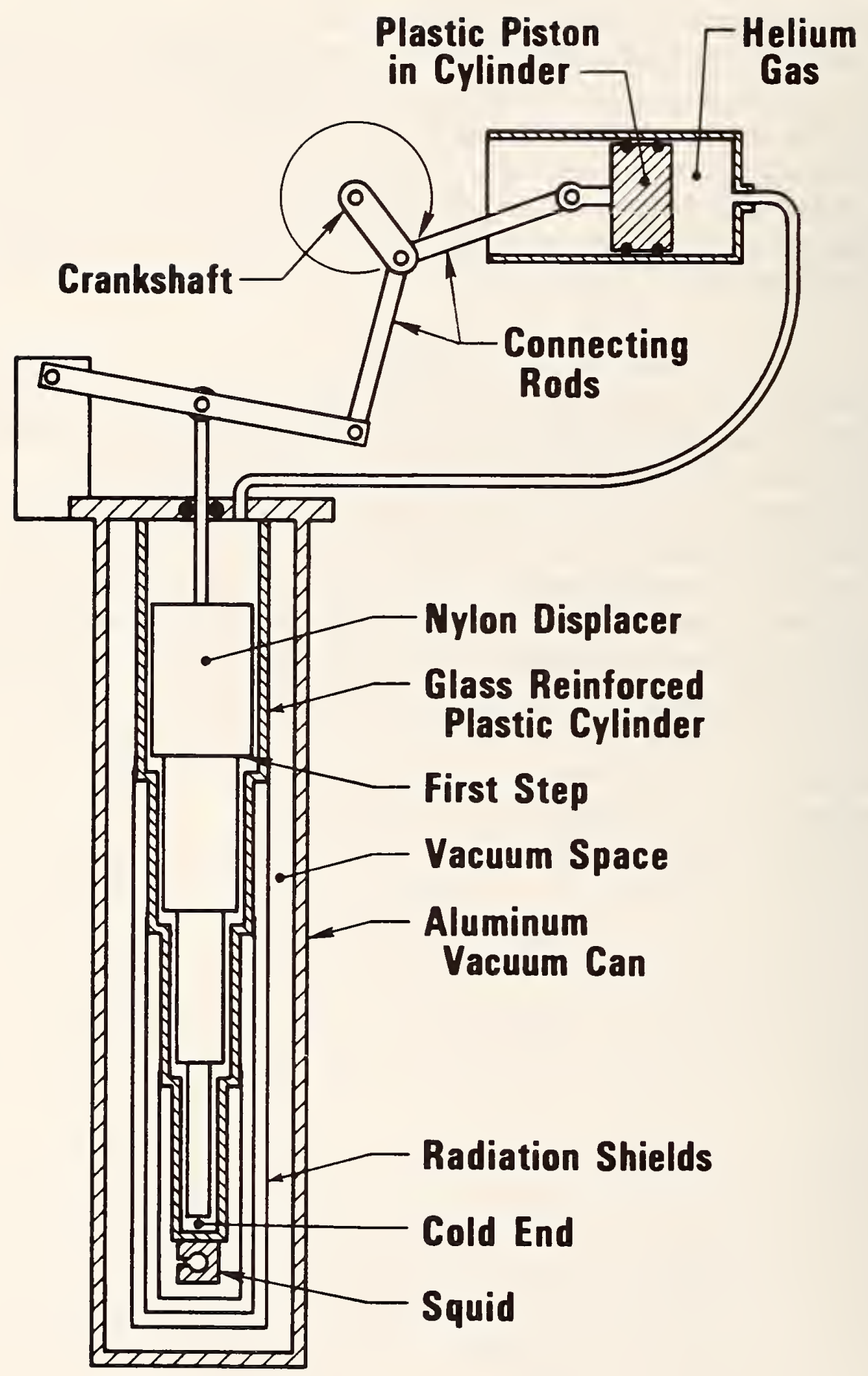

Figure 1. Schematic diagram of low-power SQUID cryocooler system, not to scale. Displacer dimensions and other data are given in text. 


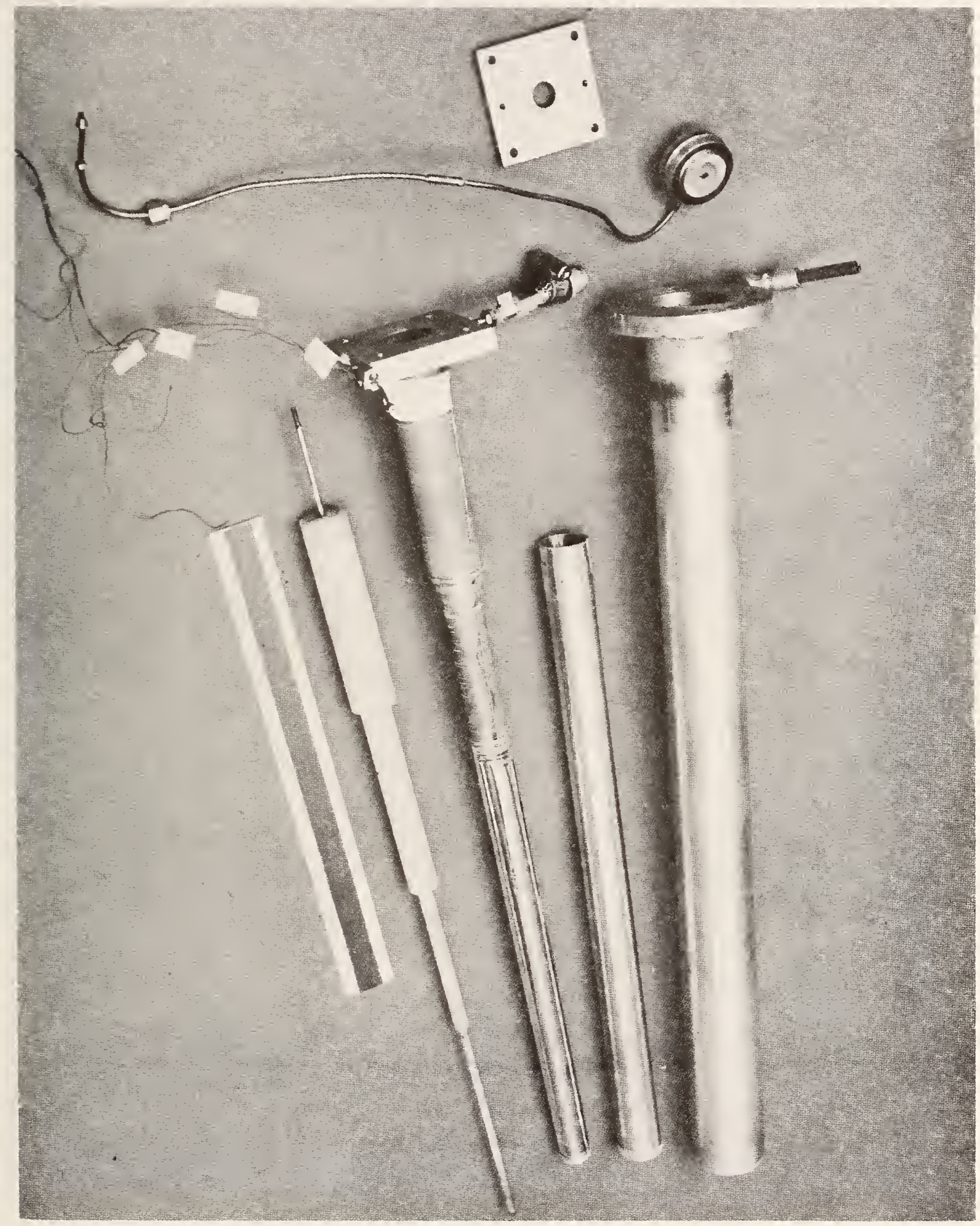

Figure 2. Cold cylinder and displacer of $8.5 \mathrm{~K}$ cryocooler. Left to right: $30 \mathrm{~cm}$ scale, nylon displacer, glass-reinforced epoxy cylinder with inner radiation shields in place, outer radiation shield, and vacuum jacket. 


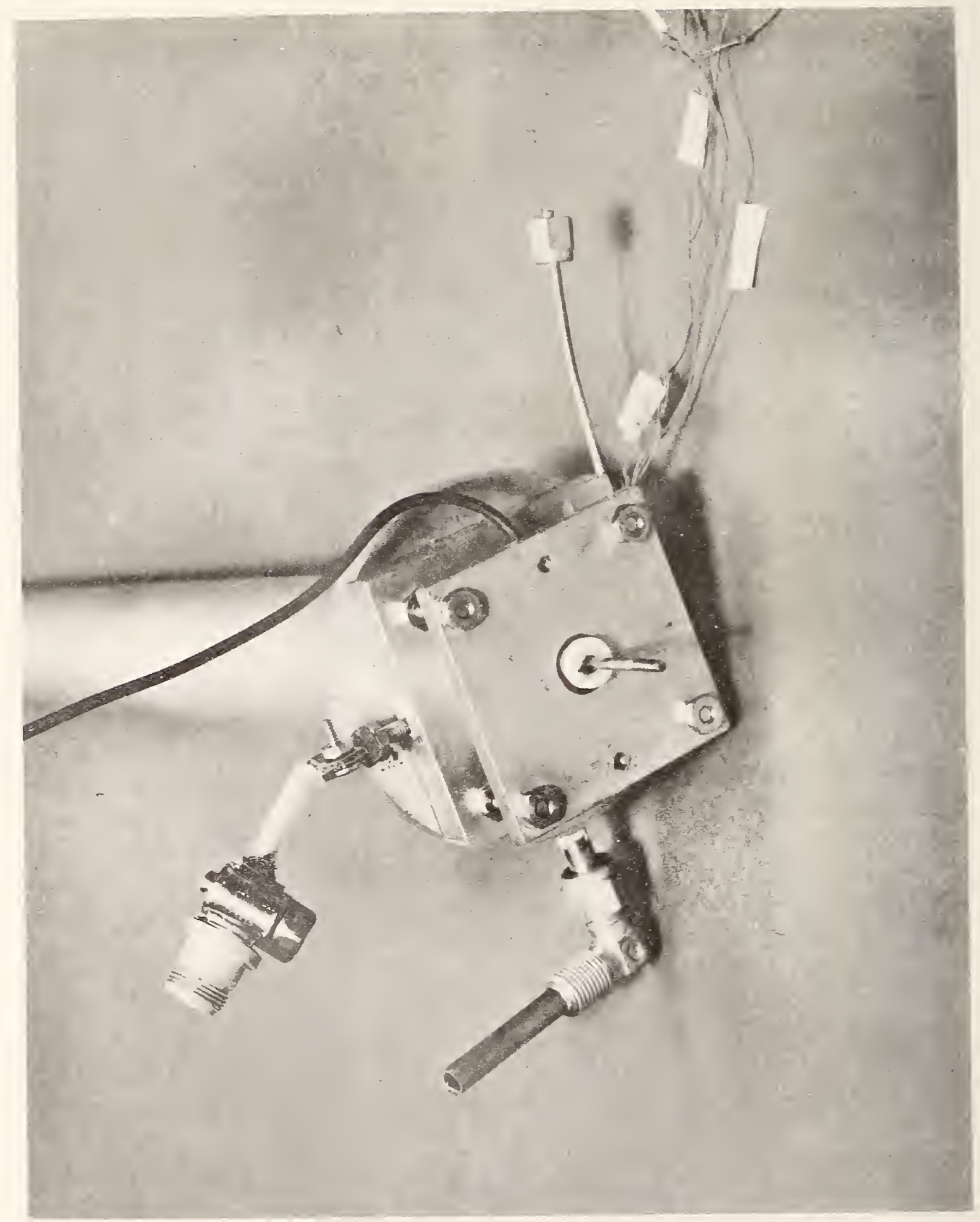

Figure 3. Room-temperature end of cold cylinder assembly. Clockwise from left: gas line to working cylinder, gas-thermometer line, thermocouple wires, vacuum connection, and $r f$ SQUID connector. 
"Applications of Closed-Cycle Cryocoolers to Small Superconducting Devices," NBS Special Publication SP-508 (J. E. Zimmerman and T. M. Flynn, eds.), Superintendent of Documents, U. S. Government Printing Office, Washington, D. C. 20234 (1978), pps. 67-73.

\section{SHUTTLE HEAT TRANSFER IN PLASTIC DISPLACERS AT LOW SPEEDS *}

Ray Radebaugh and J. E. Zimmerman

Cryogenics Division

National Bureau of Standards

Boulder, Colorado 80303

\section{ABSTRACT}

Previous analyses of shuttle heat transfer in refrigerators with displacers have neglected radial temperature gradients in the displacer or cylinder. Such analyses are valid when the gas gap is the dominant thermal resistance. We show that with plastic materials for the displacer and cylinder, shuttle heat transfer can be dominated by the thermal penetration depth of the plastic when operating at low speeds. An equation is derived for the shuttle heat transfer in such cases. Experimental data on shuttle heat transfer is obtained for temperatures down to $120 \mathrm{~K}$ for different strokes and speeds and the agreement with calculated values is good.

* Supported in part by the Naval Electronic Systems Command 


\title{
SHUTTLE HEAT TRANSFER IN PLASTIC OISPLACERS AT LOW SPEEDS
}

\author{
Ray Radebaugh and J. E. Zimnerman \\ Cryogenics Division \\ National Bureau of Standards \\ Boulder, Colorado 80303
}

\section{INTRODUCTION}

The Stirling-cycle refrigerator described previously [1] requires no net refrigeration power to keep a SQUID cold. The lowest temperature reached is the point where the total refrigeration power is cancelled by varfous heat flows to the low temperature end. These heat flows come from several sources, including radiation, heat conduction down the displacer, regenerator inefficiency, and shuttle heat transfer. Radiation heat flow to the lowest temperature can be made negligible in most cases with good shielding. For temperatures somewhat above $10 \mathrm{~K}$ the regenerator inefficiency can be made fairly small. Thus the major heat flow in much of the refrigerator is from the conduction and shuttle processes. Shuttle heat transfer occurs when the displacer, with an axial temperature gradient, reciprocates inside a cylinder with a similar temperature gradient. Because shuttle heat transfer is a major source of heat leak, it must be well characterized in order to optimize the design of the refrigerator.

\section{PREVIOUS WORK}

Zimmerman and Longsworth [2] discussed shuttle heat transfer and derived an equation for it based on a simplified theory. Their theory contained four simplifying assumptions: ( $i$ ) properties are only weak functions of temperature, (ii) heat capacity of the displacer and wall are infinite, (iii) negligible gas pressure cycling, and (iv) interaction of axial conduction effects in the displacer are neglected. For square-wave motion of the displacer they derive the equation

$$
Q_{S}=\frac{{ }_{g}{ }^{\pi D S^{2}}}{4 t} \frac{\left(T_{h}-T_{c}\right)}{L}
$$

for the shuttle heat transfer, where $k_{g}$ is the thermal conductivity of the gas, $D$ is the displacer diameter, $S$ is the stroke length, $t$ is the radial clearance, $L$ is the length of the displacer, $T_{h}$ is the hot temperature, and $T_{c}$ is the cold temperature. For sinusoidal motion the average temperature drop across the gas gap is $2 / \pi$ times that for square-wave motion. Hence for sinusoidal motion the average shuttle heat transfer becomes

$$
Q_{S}=\frac{k_{g} D S^{2}}{2 t} \frac{\left(T_{h}-T_{c}\right)}{L}
$$

More exact calculations may give different numerical doefficients, but the functional dependence of the shuttle heat loss on the various parameters will remain the same.

Harness and Meumann [3] calculated shuttle heat transfer when assumption (iv) was relaxed. The problem then becomes very complex and can be solved only by numerical integration of a second-order differential equation. Their results for the total heat flow differ from 
that of adding the static heat conduction down the displacer to the simple shuttle heat transfer equation given above. The difference ranges from 5 to $40 \%$, depending on various parameters, and is not always of the same sign.

As far as we know, attempts to solve the shuttle heat transfer problem with any of the other three assumptions relaxed has never been done. However, we were concerned that assumption (ii) was not satisfied when the displacer and wall materials were of plastic and their operation was at the low speed of about $60 \mathrm{rpm}$. When the heat capacity of the displacer and wall are assumed to be infinite, radial temperature gradients are thus neglected. In highspeed operation little heat is transferred across the gas gap in each cycle. As a result, the surface temperature of the displacer and wall remain nearly constant in time and the assumption ( $i i)$ is satisfied. Temperature cycling of the surface does become important at low speeds. The use of plastic materials instead of metal increases the amount of temperature cycling because the lower thermal conductivity decreases the thermal penetration depth.

\section{THEORY}

In order to keep the problem from becoming too complex, we first make the following assumptions: ( $i$ ) properties are nearly constant with temperature, ( $i i)$ thermal resistance of the gas gap is negligible, ( $i i i)$ the effect of gas-pressure cycling is neglected, i.e., regenerative action is perfect and heat capacity of the gas in the gap is negligible, (iv) interaction of the axial conduction effects in the displacer and wall are neglected, and (v) motion of the displacer is sinusoidal.

The shuttle heat transfer is just the net enthalpy flow resulting from the motion of the displacer. Its temperature is higher during the hot-to-cold motion than during the reverse motion so enthalpy is transported toward the cold end. The net enthalpy flow is just equal to the heat transferred to the displacer during one-half the cycle over a length equal to the stroke. To evaluate this heat transfer we first let the wall have infinite heat capacity so that it has no temperature cycling. We relax this condition later. Because of assumptions (ii) and (iii) above, we can say that the surface of the displacer takes on the temperature of the wall adjacent to it at any moment in time. The sinusoidal motion of the displacer then causes a sinusoidal temperature fluctuation of the surface, provided the axial temperature gradient is constant over the distance of the stroke. The problem of sinusoidal temperature fluctuations on the surface of a semi-infinite solid is commonly treated in texts on heat conduction. Schneider [4] treats such a problem and the total heat transferred during one-half of the cycle is given as

$$
Q=2 A \Delta T_{0} \sqrt{k C / \omega},
$$

where $A$ is the surface area, $\Delta T_{0}$ is the peak of the temperature fluctuation, $k$ is the thermal conductivity of the displacer, $C$ is its specific heat per unit volume, and $\omega$ is the frequency of oscillation. For $\dot{n}$ cycles per second $\omega=2 \pi \dot{n}$. Now $A=\pi D S$ and $\Delta T_{0}=(d T / d x)(S / 2)$. Thus the heat transfer during one-half cycle and the net enthalpy flow per cycle become 


$$
Q=D S^{2}\left(\frac{d T}{d x}\right) \sqrt{\frac{\pi k C}{2 \dot{n}}}
$$

If the wall material has the same specific heat and thermal conductivity as that of the displacer, then $\Delta T_{0}$ will be just one-half of that considered above. The average shuttle heat transfer with $\dot{n}$ cycles per second then becomes

$$
\dot{Q}_{S}=(1 / 2) \dot{n} D S^{2}\left(\frac{d T}{d x}\right) \sqrt{\frac{\pi k C}{2 \dot{n}}}=\frac{D S^{2}}{2}\left(\frac{d T}{d x}\right) \sqrt{\frac{\dot{n} \pi k C}{2}}
$$

As before, the functional dependence should be correct, but more exact calculations may give slightly different numerical coefficients.

For a thermal conductivity independent of $T$ we can write $d T / d x=\left(T_{h}-T_{c}\right) / L$. The primary difference between this shuttle heat transfer and that derived by Zimmerman and Longsworth is the $\sqrt{\dot{n}}$ dependence in the present calculation. Thus at low speeds the thermal resistance of the solid material limits the shuttle heat transfer, whereas at higher speeds the thermal resistance of the gas gap limits the shuttle heat transfer and Eq. (2) must be used. For intermediate speeds we derive the total shuttle heat transfer by considering the two thermal resistances to be in series. Hence

$$
1 / Q_{s}=1 / \mathscr{Q}_{s}^{g}+1 / \check{Q}_{s}^{s}
$$

where $\dot{Q}_{s}$ and $\dot{Q}_{s}^{s}$ are the shuttle heat transfers limited by the gas gap and by the solid material, respectively.

Equation (5) will be valid as long as the radius of the displacer and the wall thickness is somewhat greater than the thermal penetration depth in the material. The thermal penetration depth $\lambda$ is defined as that depth where the amplitude of temperature fluctuation is $1 / e$ of that at the surface and is given approximately by [4]

$$
\lambda=\sqrt{\mathrm{k} / \dot{\mathrm{n}} \pi \mathrm{C}},
$$

where $k / C$ is just the thermal diffusivity. Table 1 shows values of $k, c$, and $\lambda$ for nylon at various temperatures with $\dot{n}=1 \mathrm{~Hz}$. Values for $k$ are from Ashworth, et al. [5], and values for $C$ are estimated from two different sources $[6,7]$. The specific heat and thermal conductivity of G-10 glass-reinforced epoxy is roughly the same as that of nylon [8]. From the penetration depths given in Table 1 we see that finite size effects can be neglected in most practical cases. 
Table 1. Thermal conductivity, specific heat, and

thermal penetration depth of nylon for $\dot{n}=1 \mathrm{~Hz}$

\begin{tabular}{rccc}
\hline $\begin{array}{c}T \\
(\mathrm{k})\end{array}$ & $\begin{array}{c}\mathrm{k} \\
(\mathrm{mW} / \mathrm{cm} \mathrm{K})\end{array}$ & $\begin{array}{c}\mathrm{C}^{3} \\
\left(\mathrm{~mJ} / \mathrm{cm}^{3} \mathrm{~K}\right)\end{array}$ & $\begin{array}{c}\lambda \\
(\mathrm{mm})\end{array}$ \\
\hline 300 & 3.66 & 2000 & 0.24 \\
200 & 3.40 & 1430 & 0.28 \\
100 & 2.97 & 833 & 0.34 \\
50 & 2.19 & 440 & 0.40 \\
20 & 0.948 & 112 & 0.52 \\
10 & 0.385 & 22 & 0.75 \\
5 & 0.156 & 3.2 & 1.25 \\
\hline
\end{tabular}

\section{EXPERIMENTAL}

To our knowledge previous measurements of shuttle heat transfer have not been made. It was felt that such measurements were necessary to check the accuracy of the calculations from a simple theory. Shuttle heat transfer can be measured by first observing the warm-up rate of a known thermal mass at the cold end of the displacer cylinder. During this measurement the displacer is operating but the compressor is not. Next, the displacer is shut off and the static warm-up rate is measured. The difference in the two measurements gives the shuttle heat transfer. Effects of gas pressure cycling are determined by varying the average pressure in the system. In this paper measurements down to $120 \mathrm{~K}$ are reported and for this temperature range the gas pressure had no effect.

Measurements were made using a Stirling-cycle refrigerator with a single stage displacer. The displacer was made of nylon and the cylinder wall was of G-10 epoxy, $2.3 \mathrm{~mm}$ thick The displacer was $0.95 \mathrm{~cm}$ in diameter and $30.3 \mathrm{~cm}$ long. The speed and stroke could be varied. The radial clearance between the displacer and wall was about $0.1 \mathrm{~mm}$ at $145 \mathrm{~K}$ and $0.06 \mathrm{~mm}$ at room temperature. Some measurements were made with nitrogen gas instead of helium gas in the system to increase the thermal resistance of the gas gap.

\section{RESULTS}

Figure 1 shows the shuttle heat transfer as a function of the cold-end temperature when the warm end was at $300 \mathrm{~K}$. The calculated values consider both the gas gap and penetration depth limit using Eq. (6). Agreement between experimental and calculated values is excellent for a stroke of $1.19 \mathrm{~cm}$. For a $2.45 \mathrm{~cm}$ stroke the agreement is excellent at higher temperatures but worsens at lower temperatures. The disagreement may be a result of the temperature gradient not being constant over the stroke length or it could be a result of interaction with static thermal conduction. 


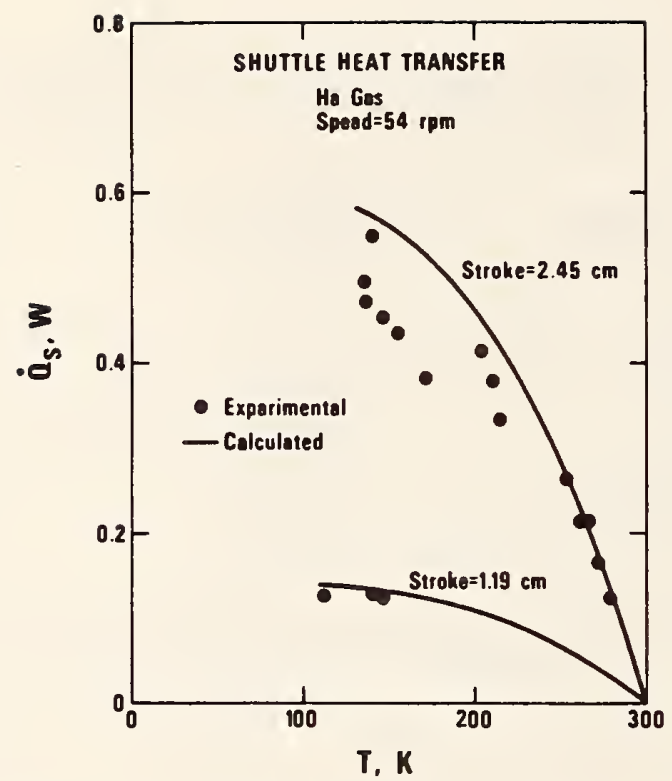

Figure 1. Measured and calculated shuttle heat transfer as a function of coldend temperature with the hot end at $300 \mathrm{~K}$.

Figure 2 shows the speed dependence of the shuttle heat transfer. This figure shows how the shuttle heat transfer gradually varies from a $i^{1 / 2}$ dependence at low speeds to an independence of $\dot{n}$ at higher speeds. The experimental results agree fairly well with calculated values for both $\mathrm{He}$ and $\mathrm{N}_{2}$ gas in the gap.

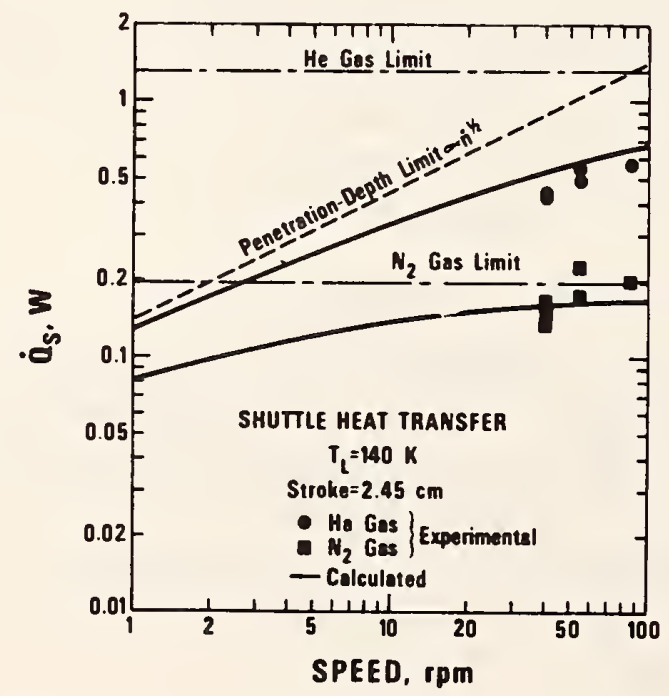

Figure 2. Measured and calculated shuttle heat transfer as a function of displacer speed for a cold-end temperature of $140 \mathrm{~K}$. 
We conclude that at least for the parameters used in this experiment, shuttle heat transfer can be estimated to within about $15 \%$ by combining the two expressions given by Eqs. (2) and (5) in the manner prescribed by Eq. (6). This imples the two thermal resistances are in series and that the necessary simplifying assumptions can be met in practice. Optimization of the parameters of the Stirling refrigerator can then be carried out analytically. In the tests done here the shuttle heat transfer was about five times the static conduction for a stroke of $1.19 \mathrm{~cm}$. These results show that a more optimum design would have a shorter stroke and larger diameter to minimize the total heat transfer. Because $k$ and $C$ are proportional to $\mathrm{T}^{3}$ at lower temperatures, the calculated values may not be in quite so good agreement with experiment at such temperatures unless Eq. (5) is integrated properly between $T_{h}$ and $T_{c}$. Measurements down to about 5-10 K would be useful and are in progress.

\section{REFERENCES}

[1] J.E. Zimmerman and Ray Radebaugh, this publication.

[2] F.J. Zimmerman and R.C. Longsworth, Adv. Cryog. Eng. 16, 342 (1970).

[3] J.B. Harness and P.E.L. Neumann, Proc. Int'l Cryog. Eng. Conf., 5th (1972) p 97.

[4] P.J. Schneider, Conduction Heat Transfer, Addison-Wesley, Cambridge, MA (1955) p 276.

[5] T. Ashworth, J.E. Loomer and M.M. Kreitman, Adv. Cryog. Eng. 18, 271 (1973).

[6] W. Reese and J.E. Tucker, J. Chem. Phys. 43, 105 (1965).

[7] B. Wunderlich, Rensselaer Polytechnic Institute Report No. TR-17, Office of Naval Research Contract Nonr-591(19) (1968).

[8] J.A. Barclay, S.S. Rosenblum and W.A. Steyert, Cryogenics 16, 539 (1976). 
XV International Congress of Refrigeration, Venice, Italy (September, 1979).

\author{
ANALYSIS OF REGENERATOR INEFFICIENCY FOR STIRLING-CYCLE * \\ REFRIGERATORS WITH PLASTIC DISPLACERS \\ Ray Radebaugh \\ Thermophysical Properties Division \\ National Buresu of Standards \\ Boulder, Colorado, U.S.A.
}

\title{
1. INTRODUCTION
}

One of the nafor sources of heat flow to the cold end of a Stirling-cycle refrigerator operating below about $15 \mathrm{~K} 1 \mathrm{~s}$ due to regenerator inefficlency. Many analyses and experiments have been performed previously, but all have dealt with the case of negligible thermal resistance internal to the regenerator material. Recently temperatures as low as $3 \mathrm{~K}$ have been reached with a Stirling-cycle refrigerator that uses fiberglass-epoxy for all the low temeprature parts./1/ Plastic regenerators - displacers are necessary for operation of SQUID magnetometers, but the Internal thermal resistance of the plastic dominates the behavior of the regenerator. Th1s paper analyzes the inefficlency of such regenerators and compares the results with experiment. In addition the effect of heat flow to the cold end caused by non-1deal gas effects ere considered.

\section{DEVELOPMENT OF MODEL}

In all of the refrigerators bullt to date with plastic displacer - regenerators, the regenerator is the annular gap between the displacer and the walls. For the $3 \mathrm{~K}$ refrigerator an additionsl annular gap outside the first cylinder was provided $/ 1 /$. The model we use to describe the regenerator behavior is one of a semi-infinite solid with the surface undergoing a cyclic temperature variation. In addition there is a temperature gradient parallel to the surface. Nusselt /2/ has considered the case of regenerators which have a finite conductivity normal to the gas flow and zero conductivity parallel to the gas flow. Of the cases considered by Nusselt this one 1s the closest to our situation except that the thermal conductivity and specific heat are strong functions of temperature. The complete set of second-order partial differential equations then become so complex that thatr solution would require considerable computer time.

In order to 8 implify the equations governing the regenerator behavior we make the following assumptions:

(1) temperature variation at the surface is sinusoidal,

(2) surface temperature 18 the gas temperature,

(3) gas volume in regenerator 18 negligible, compared with expansion volume.

(4) the sinusoldal heat flow is only in the radial direction,

(5) the expansion and compression space are 1 sothermal, 1.e., the Schmidt analysis /3/ can be used,

(6) varfous hest flows to the expansion space are independent of each other.

Asaumptions 2-4 appear to be quite ressonsble in the range of the present experiments. Assumption 1 is made since it greatly simplifies the calculations even though the siow of gas through the regenerator $1 \mathrm{~s}$ only approximately sinucoldal. For a sinusoldal temperature variation at the surface, the heat flow into (or out of) the regenerator during one half the cycle $18 / 4 /$

$$
Q=2 A \Delta T_{0} \sqrt{\mathrm{KC}_{\mathrm{v}} / 2 \pi \mathfrak{r}}
$$

For an annular gap heat can flow both outward and 1nward in the solid material and 20 the surface area $A$ should be $2 \pi \mathrm{DL}$. The amplitude of the temperature variation 
decreases with the distance from the surface. The thermal penetration depth $\lambda$ is defined as

$$
\lambda=\sqrt{\mathrm{k} / \mathrm{C}_{\mathrm{v}} \mathbf{R}^{\mathbf{L}}}
$$

where the amplitude $18 \mathrm{l} / \mathrm{e}$ of that at the surface. Thus the model considered 18 valid only for wall thickness, $d>\lambda$.

The validity of assumption 2 and of using the seut-infintte solid model for d -3 min is shown In FIg. 1 which compares the $\Delta T$ of the surface of the semi-

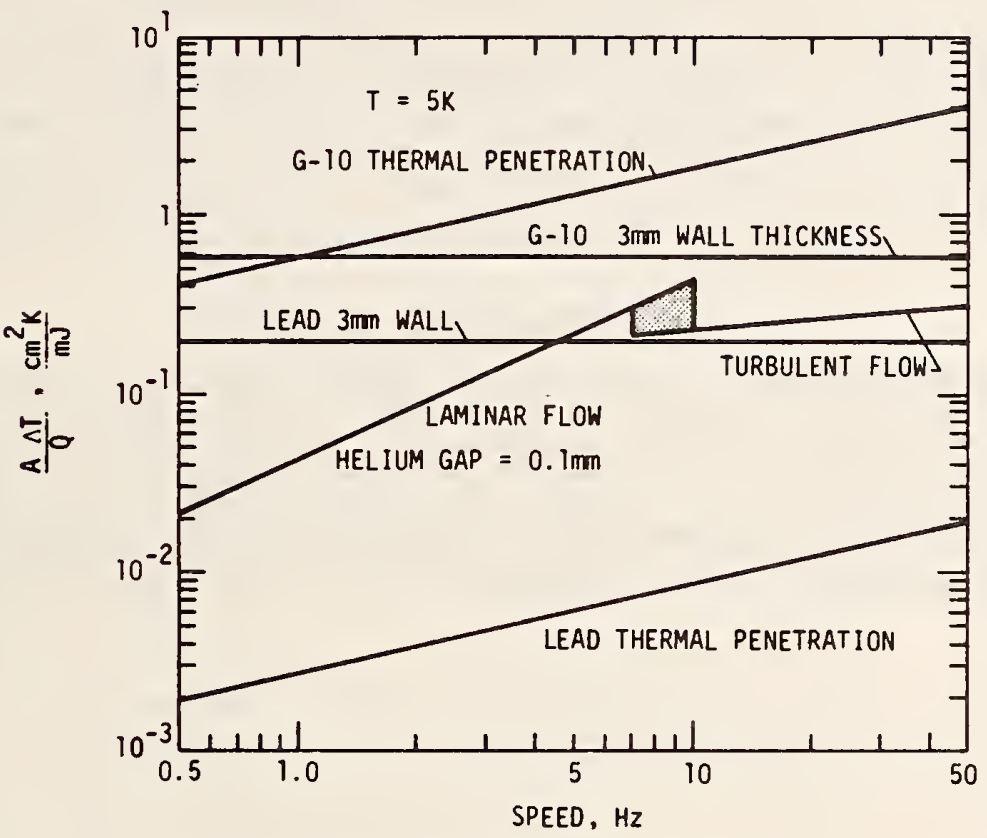

F18. 1. A comparison of the $\Delta T$ times area per unit heat flow as a function of apeed in the gas and in the solid regenerator.

Infinite solid with that due to a wall $3 \mathrm{~mm}$ thick which has $\lambda=\infty$ and with the $\Delta T$ across the hellum gas. For speeds of $1 \mathrm{hz}$ and above the dominant $\Delta T$ is due to the finite thermal penetration depth of the G-10 fiberglass epoxy. The thermal penetration depth Increases rapidly as the temperature 18 lowered and at $5 \mathrm{~K} 1 \mathrm{~s} \lambda=2.1 \mathrm{~mm}$ for a - $1 \mathrm{~Hz}$.

Beat flow into the regenerator comes from decreasing the temperature of the hellum gas. The amplitude $\Delta \mathrm{T}_{\mathrm{o}}$ is found by equating the heat flow from the gas, ${ }_{\mathrm{c}} \mathrm{p} d \mathrm{~T}$, with that in the ool1d, 1.e., Eq. (1). After some rearranging of terms, we get

$$
\Delta T_{0}=m C_{p}(d T / d x) / 4 D \sqrt{\pi k C_{v} / 2 t}
$$

Because of assumption 4 we take $d T / d x$ to be that caused by conduction heat transfer. An Iterative procedure is required to $f$ ind $\Delta T$ since the average temperature used to calculate $\mathrm{dT} / \mathrm{dx}$ depends on $\Delta \mathrm{T}$. At the cold end the regenerator temperature osc1llates between $\mathrm{T}$ and $\mathrm{T}+2 \Delta \mathrm{T}_{0}{ }^{\circ}{ }^{\circ}$ The calculated $\Delta \mathrm{T}_{0}$ usually $1 \mathrm{~s}$ less than about 0.7 $K$ for $T=5 \mathrm{R}$. The incoming gas then enters the expansion space at an average temperature of $T+\Delta T_{0}$ and thus causes an effective heat flow of

$$
\dot{Q}_{\text {reg }}=\dot{\operatorname{mim}}_{\mathrm{p}} \Delta \mathrm{T}_{0}=\dot{\mathrm{n}}\left(\mathrm{mC} \mathrm{C}_{\mathrm{p}}\right)^{2}(\mathrm{dT} / \mathrm{dx}) / 4 \mathrm{D} \sqrt{\pi k C_{\mathrm{v}} / 2 \dot{\mathrm{n}}} \text {. }
$$


The term $m C_{p} 18$ found from an integration over the crank angle using the Schmidt analysis.

The overall refigerator behavior is calculated by using the heat balance equation

$$
\dot{Q}_{\text {ref }}-\dot{q}_{\text {net }}+\dot{q}_{\text {rad }}+\dot{Q}_{c}+\dot{q}_{8}+\dot{Q}_{\text {frict }}+\dot{Q}_{\text {reg }}+\dot{Q}_{H}
$$

The shuttle heat flow, $\dot{Q}$ has been calculated prev1ously /5/ for plast1c displacers. The term $Q_{1}$ occurs in a non-1deal gas becsuse the total enthalpy flow from $T$ to $T$ is greater than enthalpy flow in the reverse direction due to the pressure variations. To calculate the minimum temperature we set $\dot{Q}_{\text {net }} 0$. The weakest assumption 185 , since $\lambda$ for hellum at $0.1 \mathrm{MPa}$ is only $0.2 \mathrm{~mm}$ at $5 \mathrm{~K}$, but $1 \mathrm{t}$ increases to $7.7 \mathrm{~mm}$ at $300 \mathrm{~K}$. Thus nearly adlabat1c behavior occurs at $5 \mathrm{~K}$ and the Schmidt analysis for Qref would be too high by possibly a factor or two. Since the Schmidt analysis 18 also used to find the mass flow, the terms $\dot{Q}_{\text {reg }}$ and $\dot{Q}_{H}$ would also be tgo high by roughly the same factor. For $Q_{\text {. }}=0$ and $T^{r} \xi_{7} \mathrm{~K}$ the terms $Q_{\text {reg }}$ and $Q_{H}$ dominate the others 80 assumption 5 may be satisfactory when calculating the minimum temperature.

\section{COMPARISON WITH EXPERIMENT}

Calculated behavior 18 compared with the experimental behavior of the refrigerator described by 2 immerman and Sullivan $/ 1 /$. The calculations for the minimum temperature were done by setting inet - 0 and calculating the required regenerator length for different expansion opace temperatures. Because of the 18othermal assumption the calculations for $\mathrm{Py}$, cannot be done in the two fluid region reached in the experiments. The term in increases rapidiy as liquid is formed but only $3 x$ liquid was produced in the experiments. Thus extrapolation to the lower temperatures should be reasonable. Figure 2 shows calculated regenerator

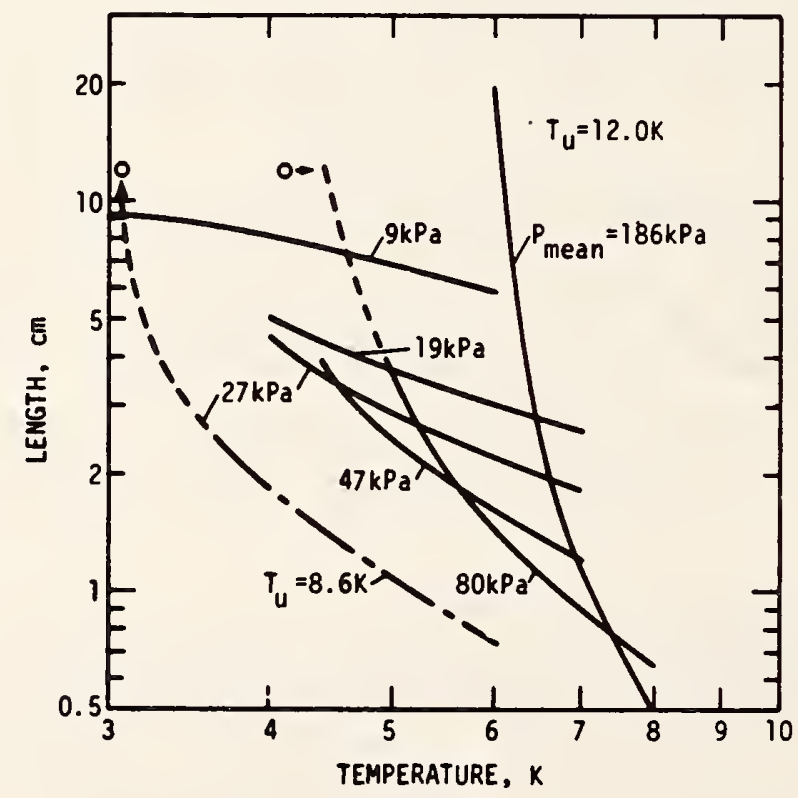

718. 2. Calculated regenerator langths as a function of the expansion space temperature. All calculated curves are for $T_{u}=12 \mathrm{~K}$ excep $r$ the lowest curve. The experimental points at $\mathrm{L}=12 \mathrm{~cm}$ are for $\mathrm{T}_{\mathrm{U}}=8.6$ $R$, $P_{\text {mean }}-27 \mathrm{kPa}$ and for $T_{U}=12 \mathrm{~K}, P_{\text {mean }}-80 \mathrm{kPa}$. 
lengths for different expansion space temperatures and pressures. W1th $T_{4}=8.6$ $\mathrm{K}, \mathrm{P}=27 \mathrm{kPa}$, and $\mathrm{L}=12 \mathrm{~cm}$ the experimental result of $3.1 \mathrm{~K}$ is conslatent with the extrapolated value from calculations. For $T_{u}=12 \mathrm{~K}$, $P_{\operatorname{mean}}=80 \mathrm{kPa}$, and $L=12 \mathrm{~cm}$ the calculated value of $4.4 \mathrm{~K}$ is in good agreement with the experinental value of $4.1 \mathrm{~K}$. The optimum pressure for $T=4 \mathrm{~K}$ and $T_{U}=12 \mathrm{~K} 1 \mathrm{~s}$ about $27 \mathrm{kPa}$ according to Fig. 2. The optimum diameter and strq̧ke are about 6 and 10 an respectively for a displaced cold volume of $0.3 \mathrm{~cm}^{3}$.

Figure 3 shows a comparison of $\dot{Q}_{\text {net }}$ for experiment and theory: The winimus temperatures agree well but the agreement in $Q_{\text {net }}$ deteriorates as $Q_{\text {net }}$ Increases, presumably due to the adiabatic behaylor. Flgure 4 showa the magnitude of the varlous heat flow terms. Note that $\dot{Q}_{\text {reg }} \propto \dot{\mathrm{n}}^{392} \mathrm{P}^{2}$ from Eq. (4), since a $\propto$ P. Figure 4 shows only a slight deviation from $\mathrm{p}^{2}$ behavior becauge of the preseure dependence of $C_{p}$. For $C_{p}$ Independent of temperature $Q_{\text {reg }} a T^{-S}$ for $T \simeq 5 R$.

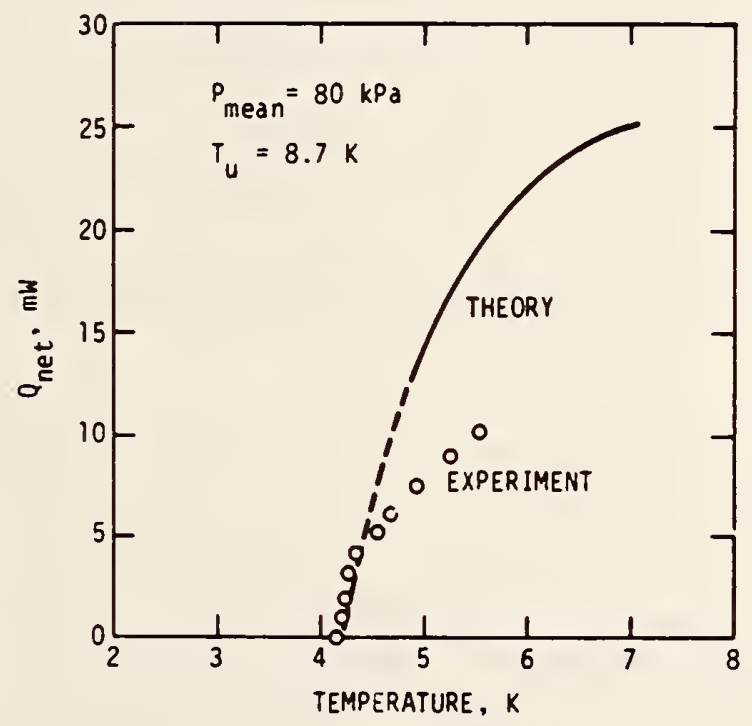

F1g. 3. A comparison of the theoretical and experimental net refrigeration powers as a function of temperature.

\section{DISCUSSION}

Because $\dot{Q}_{\text {ref }} \alpha \dot{n} p$ and $\dot{Q}_{\text {reg }} \alpha \dot{n}^{3 / 2} p^{2}$ we see imedlately the advantage of ueing low pressures and low speeds to improve the overall performance of the low temperature stage where $Q_{r e g}$ becomes large. Further improvements in the regenerator should be directed toward increasing $C_{y}$ but keeping $k$ small

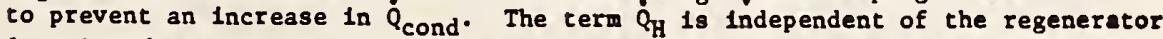
length and go it can easily dominate the heat flow for long regenerators. Fig. 4

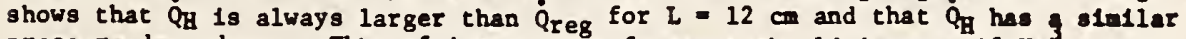
pressure dependence. The refr1gerator performance should improve if He were used because $Q_{H}$ would be reduced. The model discussed here appears to predict the minimum temperature to within about 10\%. Thus for $\dot{q}_{\text {net }}=0$ this model can be used to optimize the design of such refigerators. 


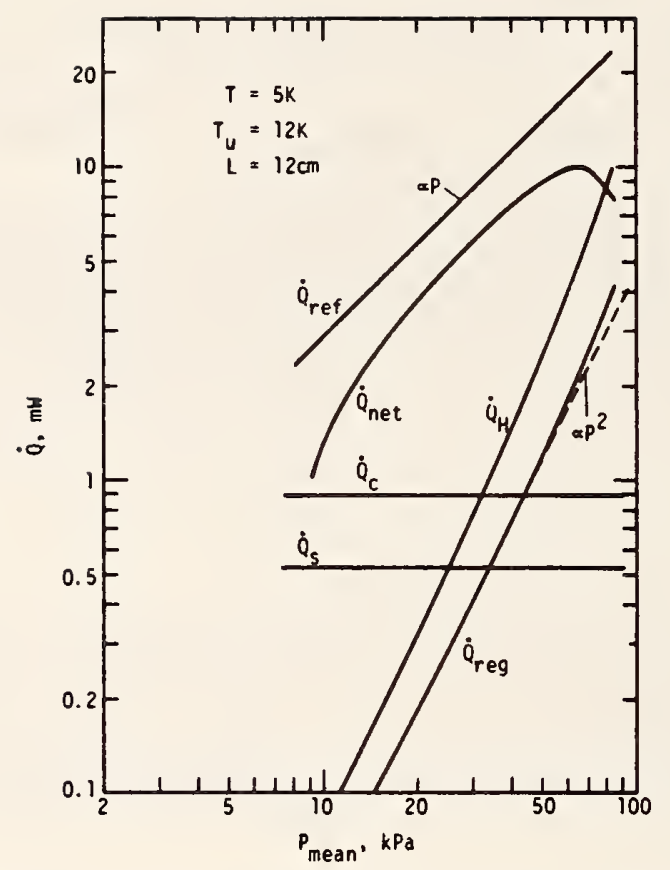

F1g. 4. Behavior of the vartous heat flow terms as a function of pressure for $\mathrm{L}=12 \mathrm{~cm}$.

NOMENCLATURE

A

surface area for heat transfer

$C_{p}$ specific heat of hellum at constant pressure

$C_{v} \quad$ volumetric specific heat of the regenerator material

D dlameter of the annular gap in the regenerator

$k$ thermal conductivity of the regenerator material

$\mathrm{L}$ length of the regenerator

m total hellum mass flow into the expansion space

i operating speed of refrigerator

$P$ pressure of hellum gas

Q cumulative heat transfer

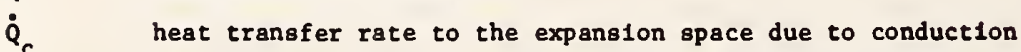

$\dot{Q}_{\text {frict }}$ heat transfer rate to the expansion space due to friction

$\dot{Q}_{H}$ heat transfer rate to the expansion space due to enthalpy Imbalance

$\dot{Q}_{\text {net }}$ net heat transfer rate to the expansion space

$\dot{Q}_{\text {rad }}^{\text {net }}$

$\dot{Q}$ rad

heat transfer rate to the expansion space due to radiation total refrigeration rate in the expansion space

effective heat transfer rate to the expansion space due to regenerator Inefficlency 


$\dot{Q}_{8}$ heat transfer rate to the expansion space due to shuttle transfer
$\mathrm{T} / 5 /$
$\mathrm{T}_{\mathrm{u}}$ temperature of the expansion space
$\Delta \mathrm{T}_{0}$ temperature of the hot end of the regenerator
$\mathrm{dT} / \mathrm{dx} \quad$ temperature gradient $1 \mathrm{n}$ the regenerator in the direction of gas flow
$\lambda \quad$ thermal penetration depth due to surface temperature varlations

\section{REFERENCES}

*ork supported by U.S. Naval A1r Systems Comand. Contribution of the U.S. Government, not subject to copyright.

1. J. B. 21merman and D. B. Sullivan, "A M1lliwatt St1rling Cryocooler for Temperatures Below $4 \mathrm{~K}^{\prime \prime}$, Cryogen1c8, March (1979), to be published; D. B. Sullivan and J. E. Z1merman, "Very-Low-Power St1rling Cryocoolers Using Plastic and Composite Materials", these proceedings, and references cited therein.

2. W. Nusselt, 2 . Ver deut. Ing. 71,85 (1927), and M. Jakob, Heat Transfer, vol. II. J. W1ley \& Sons, New York (1957), p. 288.

3. G. Walker, St1rling-Cycle Machines, Clarendon Press, Oxford (1973), p. 24.

4. P. J. Schne1der, Conduction Heat Transfer, Add1son-Wesley, Cambr1dge, MA (1955), P. 280.

5. R. Radebaugh and J. R. Z1merman, "Shuttle Heat Transfer in Plastic Displacers at Low Speeds", NBS Speclal Publication 508 (1978), p. 67. Avallable from U.S. Government Printing office, Washington, D.C. 20434.

\section{ANALYSE DE L'INEFFICACITE DU REFENERATEUR DES REFROIDISSEURS A CYCLE-STIRLING AVEC DEPLACEUR EN PLASTIQUE}

SOMAIRE: Ls source majeure de transferts thermiques dsns un refroidisseur a cycle-Stirling fonctionnsnt moins de $15 \mathrm{~K}$ environ, provient de l'inefficscité du régénersteur. Toutes les expériences et snalyses précédentes de l'inefficscité ont eté effectuées dsns le cas d'une résistsnce thermique interne négligesble du matériau du rógénersteur. Dsns le cas du refroidissement d'un magnétomètre SQUID, les orgsnes bssse-tempérsture du refroidisseur sont en plsstique. Donc, ls résistsnce thermique interne est ls résistsnce dominante. Cet exposé examine le trsnsfert thermique da a l'inefficscité du régénérsteur lorsque les matérisux sont a basse diffusion thermique. Dsns un tel c8s, le trsnsfert de chaleur est proportionnel a $n^{3 / 2} p^{2}$, oi $n$ est 18 vitesse et $P$ ls pression de l'helium gazeux. Du fsit que le tsux de refroidissement est proportionnel a nip, une réduction de ls vitesse et ce la pression réduit ls tempérstufe minimum jusqu' à ce que le trsnsfert thermique de vs-et-vient, proportionnnel a $\dot{n}^{1 / 2}$, et le trsnsfert thermique de conduction, deviennent importsnts. Une compsrsison svec les résultsts expérimentsux à des températurea de l'ordre de 3K, sers fnurnie. 


\title{
A milliwatt Stirling cryocooler for temperatures below $4 \mathrm{~K}$
}

\author{
J.E. Zimmerman and D.B: Sullivan
}

We have built a single-stage, I win-piston St irling machine' producing several $\mathrm{mW}$ of refrigeration at temperatures in the range of 3 to $4 \mathrm{~K}$, and rejecting 50 to $100 \mathrm{nW}$ at teniperatures of $81014 \mathrm{~K}$. The working fluid is helium at subatmospheric average pressure. During operation, several $\mathrm{mm}^{3}$ of liquid helium collects in the dead space at the cold end, and is present throughout the operating cycle. Thus the operating temperature is essentially the equilibrium vapour pressure of helium at the average pressure - for example. $3.1 \mathrm{~K}$ was achieved with an average pressure of $0.027 \mathrm{MPa}$. and $4.0 \mathrm{~K}$ with an average pressure of $0.08 \mathrm{MPa}$. The hot end temperatures were $8.6 \mathrm{~K}$ and $12 \mathrm{~K}$ respectively.

The cold parts of the unit shown in Fig. 1 consist of two concentric pistons and a stationary outer cylinder, all three members being made of conımercially available spun-glass epoxy ' $G-10$ ' rod and tubing requiring no machining by us. Small radial clearances and large length-to-diancter ratios prevent significant gas leakage between the ends of the pistons at room temperature. Thus, the working fluid provides gas lubrication between adjacent surfaces, and no solid sliding seals (ie piston rings) are required. The two pistons are driven $90^{\circ}$ out of phase, with a stroke of $1 \mathrm{cml}$, by connecting rods and a crankshaft (not shown) at room temperature. The lat ter are enclosed in a metal housing ( $\sim 1$ \& volunie) which is filled with helium gas to the desired pressurc. The only moving seal required is an O-ring around the crankshaft end which extends out side the housing to an electric drive motor. Owing to the reduced viscosity of helium at low temperature, there should be little pressure drop across the regenerator gaps so the pressures in the expansion and compression spaces should be essentially equal at all times. In principle the motor could also be enclosed in the metal housing and no scals at all would be necessary. Since there are no sliding seals on the pistons, the average pressure in the cylinders is identical to that in the upper housing. Most of our data were taken with an operating speed of $1 \mathrm{~Hz}$

For the experiments reported in this note we maintained the hot flange on the upper cylinder at temperatures in the range of 8 to $14 \mathrm{~K}$ by means of a weak thermal link to a liquid helium bath and an electrical heater mounted on the hot nange. The temperature of the hot nange was controlled simply by varying the heater power. The boil-off rate from the helium bath was used as a measure of the heat input to the system and, in particular, we used changes in the boil-off rate to estimate the heat ejected from the hot end of the refrigerator during operation.

The authors ore at the Electromagnetic Technology Division, Nationel Bureau of Standards, Boulder, CO 80303 , USA. Received 9 January 1979.
As shown in Fig. 1, we incorporated a pair of universal joints in the central piston rod at the level of the conmression space. This allows the lower part of the piston to centre itself in the cylinder. Hithout this feature. slight nisalignment of the cylinder surfaces above and helow the compression space caused those surfaces to bear against the sides of the piston. The associated frictional hesting degraded the expected performance considerably.

Another feature, which nay be inportunt, shown in Fig. 1. is the 'stationary insert' around the lower piston. This miser is a piece of the same material as the upper piston. llaving equal annular gaps at both the inner and outer surfaces of the insert provides two parallel paths for fluid thow hetween the expansion and compression spaces, giving a lolial area

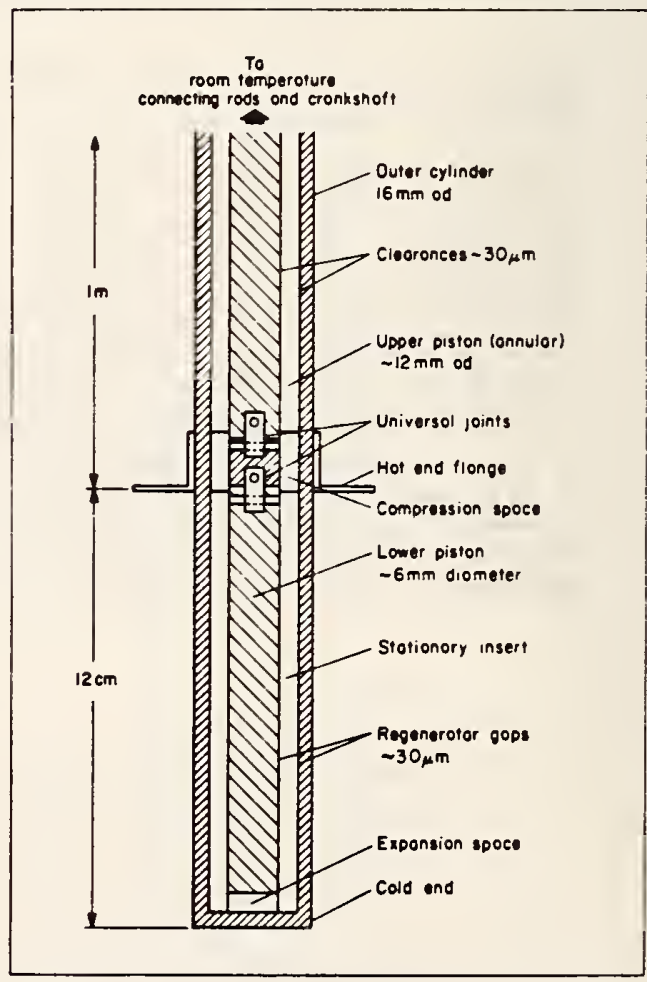

Fig 1 Sketch of single-stage Stirling cryo-cooler with approximate dimensions 


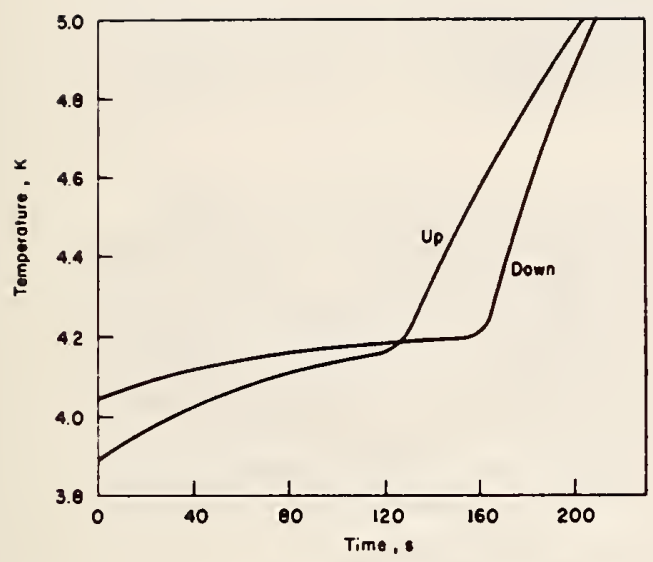

Fig 2 Temperature vs time of the cold end with the cryocooler stopped at maximum expansion llower piston "up I bnd minimum expansion (lower piston 'down')

for regenerative heat exchange three times the area of the inner gap alune. At the I $\mathrm{Hz}$ operating speed, we estimate that heat diffuses into the material less than I $\mathrm{mm}$, so effectively the insert provides three times the regenerator heat capacity. In order to analy'se the effectiveness of this particular feature, one needs to know the heat capacity and thermal cunductivity of the material, which we have measured and will report separately.

With the upper end temperature at $8.7 \mathrm{~K}$, the ref rigerat or maintained a temperature of $4 \mathrm{~K}$ at an operating pressure of $0.08 \mathrm{MPa}$. but there was some reserve couling capacity. The operating temperature was limited by the pressure of the liquid phase. Heat was added at the bottom end to determine the reserve cuoling capacity. The system maintained liquid helium at $4.0 \mathrm{~K}$ up to a heat input of $4 \mathrm{~mW}$ at which point the system reverted to single phase gas operation. At $9 \mathrm{~mW}$ input power the temperature ruse to $5.5 \mathrm{~K}$. The Carnot coefficient for the cooler is $4 /(8.7-4)=0.85$. From changes in the helium boil-off rate. we estimated that the licat cjected at the hot end is about $50 \mathrm{~mW}$. Thus the actual refrigeration of $4 \mathrm{niW}$ at $4 \mathrm{~K}$ is a reasonable fraction of the theoretical cooling power $(50 \mathrm{~mW} \times 0.85=42.5 \mathrm{~mW})$.

A calculation of the work input yields a number of the same order as the heat ejected, but not inuch information can be elicited from this since the two plase nature of the refrigerator operation complicates the analysis.
Fig. 2 shows two separate warming curves for the cold end of the refrige ratur. recurded immediately after the drive mutor was switched off. These demonstrate the presenie of liquid helium in the system. The temperature rises gradually at first since the evaporation of the liquid increases the overall sy'stem pressure. After several minutes the temperature increases more rapidly, indicating evaporation of the last bit of liquid. The total quantity of liquid helium present in the system can be cstimated from the pressure changc during the slow portion of warm-up of the botton end and comes out to be $\sim 0.02 \mathrm{~cm}^{3}$. The two curves currespund to stopping the refrigeration cycle at iwo different positions separated by $180^{\circ}$. The first portions of the curves are similar in shape although the temperatures are difierent. The time interial required for all the liquid to evaporate varies because a different quantity of liquid is present during each part of the cycle. The variation in slope beyond the break point indicates different heat capacities at the buttom end. that is, if the bottom piston is in its uppermost position then the heat capacity of the extra vapour reduces the warming rate. This is a clear indication that the heat capacity of the vapour in the expansion space is significantly greater than that of the G-10 epoxy. the implication being that the additional channel for regenerative heat exclange provided by the stationary inset maly be an essential feature.

We helieve this is the first time that a Surling machine operating as a refrigerat or has been shown to liquefy the working fluid (helium or any other gas). and the temperature achicved is ahout a factur of $t$ wo lower than has heen reported previously for snlall Stirling. Giffurd-McMdalion, and Vuilleumier machincs. ${ }^{2}$ A machine of this I! pe appears to be a practical alternative io a Joulc-Thomsun expiansion systen for the final stuge of a smab helium liquetier or iryoivoler.

The heat load that our particular machine would impose on the higher temperature stage (at 8 to / $4 \mathrm{~K}$. for example). is low enough that the total system should require an order of magnitude lower mechanical power input than any existing small cryocooler.

This work was supported by the Naval Air Systems Commind.

\section{References}

1 Walker, Ci Stirling-Crcle Machines Oaford I'niversity Près (1973)

2 Zimmerman, J.E, Flynn. T.M. NBS Special Publicaluon SP-508. National Bureau of Siandards (1977), 
"Future Trends in Superconductive Electronics," (B. S. Deaver, Jr., C. M. Falco, J. Harris, and S. W. Wolf, eds.) AIP Conference Proceedings Number 44, New York, American Institute of Physics (1978), pps. $412-420$.

\title{
CRYOCOOLERS FOR SUPERCONDUCTIVE ELECTRONICS*
}

\author{
J. E. Zimmerman \\ Cryogenics Division \\ National Bureau of Standards \\ Boulder, Colorado 80303
}

\begin{abstract}
Superconductive electronic devices are generally operated and will continue to be operated in liquid-helfum cryostats. Appropriate small closed-cycle cryocoolers for these devices do not exist. Development of cryocoolers, including efficient use of conventional gas refrigeration cycles and innovative thoughts on new and unconventlonal methods, could greatly enhance the usefulness of smal1-scale superconductive electronics. Large cryocoolers are already we11developed and can be adapted to the requirements of large-scale systems, such as computers, requiring a watt or more of refrigeration.
\end{abstract}

\section{INTRODUCTION}

In this paper I will refer repeatedly to two sources ${ }^{1}, 2$. Ref. 1 is the proceedings of a 1977 conference on "Applications of Closedcycle Cryocoolers to Small Superconducting Devices," and is publicly avallable. Ref. 2 is the proceedings of a 1973 conference on "ClosedCycle Cryocoolers," and is available for "limtted distribution." A third signiflcant source of information and speculation is the proceedings of the 1975 Navy Study on Superconductive Electronics. ${ }^{3}$ Other useful and readily available references are the proceedings of the 1976 SQUID conference ${ }^{4}$ and the proceedings of the 1976 NATO study on small superconducting devices ${ }^{5}$.

This author's demonstrated qualifications as an authority on cryocoolers consist of his having built (1) in 1949, a Simon singleexpansion hellum liquefier, a mechantsm which at the same time was made obsolete by the Collins Hellum Cryostat, (2) a few years later, a cyclic magnetic refrigerator for temperatures down to $0.1 \mathrm{~K}$, a machine which was immediately replaced by the helium dilution refrigerator, and (3) recently, a laboratory-model Stirling cryocooler specially adapted to operate a SQUID magnetometer. The trend established by the earller work probably has been reversed by the recent achlevement, since Stirling machines are now belng extensively developed both for pumping heat and for doing work, and this conference is evidence that the SQUID and other superconductive devices are seen as having great future potential. In fact, it should also be pointed

*Contribution of the National Bureau of Standards

Not subject to copyright.

Support in part by the Office of Naval Research

and the Naval Alr Systems Command 
out that cyclic magnetic cooling systems are currently being developed by Steyert and others for temperatures between 1 and $300 \mathrm{~K}$, and $1 \mathrm{t}$ is suggested below that the single-expansion process of 11quefying hellum may st1ll be of some use in certain systems (p.81 and p.49, ref. 1).

Very few closed-cycle cryocoolers are now in use, or have ever been used, to operate superconducting devices. The reason for this became evident in 1947 or shortly thereafter, when the Collins Helium Cryostat became generally available. This machine was advertised and sold as a "cryostat", a device for maintaining a constant low temperature. Very quickly it became obvious that the machine was more cost-effective if used not as a cryostat but as a liquefier, for servicing any number of separate small liquid-hellum evaporative cryostats.

\section{ESSENTIAL HEAT INPUTS INTO CRYOSTATS}

With only a few isolated exceptions, (e.g. Hartwig. p.135, ref. 1) liquid-helium cryostats have been used for all research in superconductivity and superconductive devices up to the present. Now, however, the beginning of change is apparent. As superconducting devices are found to be useful for a variety of purposes unrelated to low-temperature physics, users are searching for ways of reducing the cost and inconvenience of 11quid-helium cryogenics. One approach is to make dewars larger and more efficient, with hold times of the order of $10^{3}$ to $10^{4}$ hours (e.g. the 180-11ter VLF SQUID cryostat of Dinger et al., with a hold time of 100 days $^{6}$, the 800-11ter Infrared Astronomy Satellite (IRAS), with a projected hold time of one year', and a similar 800-11ter satellite for the Stanford relativity experiment, projected to operate one year in orbit at $2 \mathrm{~K}^{8}$. More Indicative of the nature of things to come, we hope, are the systems in which a cryocooler is incorporated into a medium-sized 11quid-helium dewar to cool one or more radiation shields and greatly reduce the evaporation rate. Temperatures well below $20 \mathrm{~K}$ can be maintained with avallable small commercial coolers. Black-body radiation at $20 \mathrm{~K}$ is only 9.1 $\mathrm{mW} / \mathrm{m}^{2}$, and if this figure can be reduced by a factor of 100 by the use of highly reflecting surfaces, giving $91 \mu \mathrm{W} / \mathrm{m}^{2}$, the corresponding evaporation rate due to radiated heat is $0.0031 \mathrm{l} /$ day $-\mathrm{m}^{2}$. A spherical container with a surface area of $1 \mathrm{~m}^{2}$ would hold 95 liters, giving a t1me of $3 \times 10^{4}$ days ( 80 years) for complete evaporation. In most practical cases, with a radiation shleld at $20 \mathrm{~K}$ and a hellum bath at $4 \mathrm{~K}$, the main heat input will be conduction along mechanical supports and electrical leads, but even so, the heat leak can in principle be made very small. If a 95-1iter container is supported by spun-glass cords, $10 \mathrm{~cm}$ long, calculated to withstand $100 \mathrm{G}$ loading in three orthogonal directions (six cords), and assuming a total 
mass twice that of the helium itself, the heat leak is calculated to be $2 \mathrm{~mW}$, giving a hold time somewhat less than 4 years ( 40 years for 10G loading). The enthalpy change of hellum between 4 and $20 \mathrm{~K}$ is about 4 times the heat of vaporization, so if the vapor can be used to cool the supports, this evaporation rate might be reduced considerably, at the expense of geometric complexity. Size of electrical leads depends upon the particular devices in the cryostat. Let us consider (as we often do) an If SQUID and suppose that the rf line to it is a thin-film stripline with a parallel dc resistance of $0.1 \Omega$ between 4 and 20K. Invoking the Wiedemann-Franz law leads to a heat leak of about $45 \mathrm{wW}$, which is smaller than any of the other heat leaks calculated above.

This exercise in arithmetic is highly idealized, but it does indicate the possibility of greatly reducing the evaporation rate of liquid helium by using a cryocooler in a carefully-designed system. A 70-11ter cryostat incorporating a cryocooler to maintain the inner radiation shield at $15 \mathrm{~K}$ has been bullt by a comerclal firm (W. Goree, private communiation). This system demonstrated a hold time greater than one year ( $0.19 \mathrm{l} /$ day evaporation rate). The heat leak into the hellum, about $5.5 \mathrm{~mW}$, was calculated to be due almost entirely to the $75 \mathrm{~mm}$-diameter stainless steel neck tube, which provided easy access to the hellum bath and also served as mechanical support for the reservoir.

Conventional liquid-helium cryostats, such as those mentioned above, without liquid-nitrogen jackets, typically evaporate from one to several liters per day. One liter per day indicates a heat input into the liquid of $30 \mathrm{~mW}$. The total enthalpy change of the vapor between 4 and $300 \mathrm{~K}$ is roughly 70 times the heat of vaporization. The cooling capacity of the vapor, proportional to the specific heat $\mathrm{CP}$, is nearly independent of temperature over most of the range. This is not, in general, the optimum distribution of cooling capacity vs. temperature. A closed-cycle cryocooler with the optimum distribution is therefore, in principle, more efficient than an evaporative cryostat. Related to this is the important point that, for many small low-power superconducting devices, the lion's share of the cooling capacity is required at higher temperatures to intercept heat radiated and conducted from room temperature. To specify the performance of a cryocooler only in terms of its cooling capacity at the cold end, as is commonly done (e.g. p.iv, ref. 3), is therefore misleading.

\section{CLOSED-CYCLE CRYOCOOLERS VS EVAPORATIVE CRYOSTATS}

The relative merits of closed-cycle cryocoolers and evaporative cryostats have been debated many times. In 1966, Winters and Snow showed that an evaporative cryostat is economically preferable to a 
cryocooler for a heat load of $100 \mathrm{~W}$ ( $3300 \mathrm{l} /$ day evaporation rate) if the duty cycle is less than 95 hours per year for 10 years ${ }^{9}$. If one may extrapolate from this by 4 or 5 orders of magnitude, 1t might be concluded that a cryocooler could not possibly be economical at milliwatt refrigeration levels. Nevertheless, economic analyses are imperfect at best, and may change with time as the data base changes. One such change now occurring is that superconductive electronics is becoming a widely used technology with large duty cycles. Another change which one may expect in the future is that helium gas will become more expensive as the supply runs out. There is also a possibility that inventive genius may change the economics of small cryocoolers by discovering better and simpler refrigeration methods (see for example W. A. Little, this conference, also R. Radebaugh, p.7, W. A. Little, P. 74 , W. A. Steyert, p.81, R. Radebaugh, P.93, and J. E. Zimmerman and R. Radebaugh, p.49, ref. 1). A factor which might significantly affect the economics of cryocoolers is the development of high- $T_{c}$ devices (M. Beasley, this conference and $\mathrm{p}$. 167 , ref. 1).

\section{COST AND RELIABILITY OF CRYOCOOLERS}

Data on cost and physical characteristics of practically all cryocoolers built up to 1973 have been collected by Strobridge ${ }^{10}$. More data on small cryocoolers, primarily for infrared sensors and space applications at temperatures of 10 to $100 \mathrm{~K}$, are given in reference 2 (W.S. Sims, . . 1, V. C. Johnson, P. 11, and W. D. Clarke III, p. 17). This reference provides interesting information ( .15 ) such as that the Air Force had expended over $9 \times 10^{6}$ dollars, as of 1973, on two objectives (1) "decrease the cost of ownership for airborne cryogenic coolers, and (2) "Increase the unattended life of cryogenic coolers for spacecraft to something in excess of three years." The second objective had not, and probably has not yet, been achieved. The reference contains only one paper relating to cryocooler technology for elemental superconductors (W. Lawless, p 417, ref. 2, see also R. Radebaugh, p.7 and p.93, ref. 1) but several papers (e.g. B. Renyer, p 147, ref. 2) describe cryocoolers capable of cooling high-T devices. Reference 2 also contains some speculation on cost of cryocoolers. S. Horn et al., p. 73, and W. Sims, p. 1 , ref. 2, have estimated prices of 1000 to 2000 dollars for the very simplest (Vuilleumier) single-stage miniature $77 \mathrm{~K}$ closed-cycle cryocoolers, in lots of 1000. Miniature multistage Stirling and Vullleumler machines for low temperatures, 6 to $8 \mathrm{~K}$, should not necessarily cost much more than this, since, by using the steppeddisplacer principle of Fokker and Kohler ${ }^{11}$, the number of essential moving parts (approximately 5) is precisely the same as for a singlestage machine, and only the displacer geometry is more complicated. Other Information of general interest from reference 2 are the comments on reliability of closed-cycle cryocoolers. W.Clarke III, p.17, stated that "fallures occur between 120 and 300 hours of 
cooler operating time on all Alr Force airborne systems," which includes Joule-Thomson, Stirling, and Gifford-McMahon cryocoolers. Chell1s, p.111, ref. 2, reports a mean time between failure of 20000 hours for several hundred Gifford-McMahon cryocoolers used (on the ground) to cool parametric amplifiers for satellite communications and also for military purposes. Clarke and Chellis were reporting on different systems with different reliability requirements, but the contrast is nevertheless impressive. More recent information on reliability is given by Chellis (p.109, ref. 1) on 20K machines and by Higa and Wiebe (p.99, ref. 1) on 4K machines. About 30 or 40 of the latter are in use to cool masers for deep-space probes.

\section{LARGE AND SMALL MACHINES AND APPLICA:IONS}

In the literature and in private conversation, cryocoolers with one to several watts of refrigeration at the cold end are of ten called "sma1l" or "minlature." In the context of this paper, such machines will be referred to as "large." This paper will deal primarily with concepts of cryocoolers for devices such as single Josephson junctions or small arrays of junctions whose inherent refrigeration requirements are in the sub-milliwatt level. At least one author (W. A. Little, p. 74, ref. 1) has pointed out that present commercial cryocoolers are grossly mismatched to such devices. With regard to large superconducting systems such as computers, which may actually require a watt or or more of refrigeration, present cryocooler technology (F. Chellis, P.109, R. Longsworth, p.45, W. Higa, p.99, ref. 1) should be readily adaptable, perhaps with some effort to reduce vibration levels (see for example, J. Cox and S. Wolf, p.123, ref.1). The computer application of cryocoolers was considered In detall by van de Hoeven and Anacker (p.221, ref.1), who indicate that refrigeration requirements may fall in the range of 0.1 to 10W.

A few "large" machines have been used for superconducting devices and research. The first such use was by W. Hartwig and his colleagues to cool a superconducting $\mathrm{Pb}$ cavity (for a review of this and other uses see p.135, ref. 1). Operation of a SQUID susceptometer is described by $D$. Vincent (p.131, ref. 1), and a cryocooler has been used or several years for low-temperature research by $W$. Little (private communication). Both of the latter two machines were originally provided by the Office of Naval Research (E. Edelsack) for operational evaluation with superconducting devices.

It is arguable that there is a need for small cryocoolers for such applications as SQUID magnetometers for geothermal prospecting (W.D. Stanley, p.205, ref. 1, and J. Clarke, ref. 5), gradiometers for magnetic anomaly detection 12 and for blomagnetic research (reviewed by S. Williamson and L. Kaufmann, P. 177, ref. 1), 
Josephson voltage standards (L. Holdeman and C. Chang, p.243, ref. 1), microwave and millimeter-wave detectors (J. Edrich, P. 159 and M. Nisenoff, p.221, ref. 1) and a varlety of other laboratory research instruments. There have been virtually no studies of small or miniature cryocoolers other than the recent one by $W$. A. Little on microminlaturizing the Joule-Thomson process (p.74, ref. 1 ).

As far as I am aware, the only experimental small (milliwatt) cryocooler work currently going on is that by me and my colleagues on a low-speed low-power 4-stage split Stirling machine for operating a Nb SQUID at $8.5 \mathrm{~K}$ (p.59, ref. 1). Th1s machine is unique in several respects, (1) it requires an order-of-magnitude-lower input power than comparable machines that have been bullt for the same temperature range, (2) it employs a gap regenerator, making a displacer and heat exchange mechanism of ultimate simplicity, and most important (3) nylon and epoxy-glass composite materials are used for the displacer and cylinder to minimize magnetic interference caused by moving parts near the low-temperature end.

The requirements for small cryocoolers were estimated by M. Simmonds (p. 207, ref. 1). Except for the susceptometer, his estimates of refrigeration requirements for various devices are all of the order of a few mW. Even these estimates are considerably higher than the above calculations would indicate, but it may be assumed that he is biased more toward commerclal realism than academic idealism. In any case, even if extremely low refrigeration power satisfies the steady-state requirements, cooldown times may be too long for some applications. Estimates of acceptable cooldown times and other parameters vary considerably (M. Simmonds, p.207 and M. Nisenoff, p.221, ref. 1).

\section{FUTURE TRENDS}

With regard to the future of superconductive electronics, there is a great need for intelligent but uninhibited analysis and experimentation on cooling methods for small superconducting devices and systems. Classical gas-cooling systems like Stirling and GiffordMcMahon machines are highly developed and effective for temperatures as low as 6 to $8 \mathrm{~K}$. This is tantalizingly close to the temperature range needed for most present devices. It is quite adequate for high- $T$ devices, and although the latter will undoubtedly serve some of the needs of superconductive electronics, lower-temperature cryocoolers will be essential for lower nolse devices, for devices like the super-Schottky diode ${ }^{13}$ which must operate well below the transition temperature, and to make use of elemental superconductors with various special properties. To span the temperature region from 
about $8 \mathrm{~K}$ down to 1 or $2 \mathrm{~K}$, the development of solid-state processes as alternatives to Joule-Thomson or other processes requiring compressors would be highly desirable. On the other hand, perhaps a low-temperature low-pressure stage using helium (or helium 3) gas in a Stirling cycle would be practical. For very low refrigeration power, a few $\mathrm{cm}^{3}$ of liquid helium produced by a single expansion, with a starting and shield temperature of $8 \mathrm{~K}$, could last practically forever. Longterm reliability is probably the most difficult thing to achieve with mechanical systems, so we should emulate Steyert by considering other refrigeration processes for higher temperatures as well. Even though conventional gas refrigerators may be hard to beat, there are numerous ways to improve and simplify these systems--gas bearings, thermal compressors, absorption-desorption compressors, resonant spring-mounted or free pistons and displacers driven by mechanical reactive coupling rather than by crankshafts and connecting rods, and (no doubt) hundreds of other good ideas.

Radebaugh (p.7, ref. 1) has written an unusually informative and stimulating review of refrigeration fundamentals. In addition to the commonly-used gas and gas-liquid systems, he describes a broad range of other systems, tells what kind of forces (electric, magnetic, tensile, centrifugal, etc.) are required to change the entropy, and estimates the minimum useful entropy change. An example of the many less-well-known systems is a rubber-band refrigerator, with which a modest amount of refrigeration might be obtained in the neighborhood of room temperature.

Space applications of superconductive electronics pose special problems for cryocoolers in terms of weight, power and reliability. Work on large cryocoolers (mostly classified) under the sponsorship of Wright-Patterson Air Force Base is continuing, with a multi-stage Vuilleumier cryocooler under development for launching in a spacecraft within a few years. For certain farout missions, cooling of superconducting devices by radiation into space has been suggested. I have estimated that a black aluminum sheet $1 \mathrm{~mm}$ thick, carefully shlelded from the sun and not too close to any of the planets or their satellites, and attached to the spacecraft by a practical lowconductivity suspension, should reach a temperature of 5 to $10 \mathrm{~K}$ in about 2 days. The estimate is based upon evidence that the total energy flux in interplanetary space, including zodiacal light, starlight, and cosmic background, but not including sunlight, adds up to something like 10 to $100 \mu \mathrm{W} / \mathrm{m}^{2}$. Solar energy flux at the earth's orblt is $1.3 \mathrm{~kW} / \mathrm{m}^{2}$. A number of applications of superconductive and other devices in space were reviewed by E. Tward and P. Mason and by J. Vorreiter and C. McCreight (p.227, and p.153, ref. 1). 
As for specific suggestions, there is a well-defined need for a cryocooler for a biomagnetic SQUID gradiometer. This instrument would operate in a stationary, well-controlled environment, where self-generated vibration and magnetic interference could be reduced by careful mounting and shielding techniques. If an economical and reliable cryocooler could be produced for this application, it would make magnetocardiography and pneumomagnetic measurements acceptable for general clinical use. Experience gained with such systems could lead naturally to development of portable instruments for geomagnetism and magnetic anomaly detection. Another, less demanding, application for a small cryocooler was suggested by $L$. Holdeman and $C$. Chang ( $p .243$, ref. 1), a simplified portable Josephson voltage standard. Systems such as these could serve as indicators of the viability of small cryocoolers for many other applications, and should not require large amounts of money for their development.

\section{REFERENCES}

1) Applications of Closed-Cycle Cryocoolers to Small Superconducting Devices, edited by J. E. Zimmerman and T. M. Flynn. National Bureau of Standards, Special Publication 508 (April, 1978) (in press).

2) Closed-Cycle Cryogenic Cooler Technology Applications, Vol. 1, Tech. Report AFFDL-TR-73-149. Wright-Patterson Alr Force Base (Dec. 1973).

3) 1976 Navy Study on Superconductive Electronics, edited by A. H. Silver, Office of Naval Research Report No. NR319-110 (Aug. 1976).

4) Superconducting Quantum Interference Devices and their Applications, edited by H. D. Hahlbohm and H. Lubbig. Walter de Gruyter, Berlin (1977).

5) Superconducting Machines and Devices, Vol. B1, edited by S. Foner and B. Schwartz, Plenum Press, New York (1974). Primarily on very large systems.

6) R. J. Dinger, J. R. Davis, and M. Nisenoff, NRL Memorandum Report 3256. Naval Research Laboratory. (Mar. 1976).

7) V. Vorreiter, private communication. See also ref. 8.

8) C. W. F. Everitt, et al., To Perform a Gyro Test of General Relativity. Final Report on NASA Grant 05-020-019. High Energy Physics Laboratory, Stanford University (July 1977). 
9) Technology of Liquid Hellum, edited by R. H. Kropschot, B. W. Birmingham and D. B. Mann. NBS Monograph 111. (Oct. 1968) p. 85.

10) T. R. Strobridge. Cryogenic Refrigerators - an Updated Survey. NBS Tech. Note 655 (1973).

11) G. Prast, Ph1lips Tech. Rev. 26, 1 (1965). J. Kohler, The Stirling Refrigeration Cycle. Scientific American, 242, 119 (1965).

12) W. M. Wynn, C. P. Prahm, P. J. Carroll, R. H. Clark, J. Wellhoner, and J. M. Wynn, IEEE Trans. MAG-11, 701 (1975).

13) M. McColl, M. F. Millea and A. H. S1lver, IEEE Trans. MAG-13, 221 (1977). 


\title{
Very low-power Stirling cryocoolers using plastic and composite materials
}

\author{
D. B. Sullivan and J. E. Zimmerman
}

\section{Cryocables à cycle Stirling de très faible puissance utilisant des matériaux plastiques et composites}

Une étude expérimentale a été entreprise, concernant plusieurs conceptions de refoidisseurs cryogéniques pour dispositifs supra-conducteurs ultra-sensibles. Les appareils à refroidir sont ceux qui utilisent les jonctions Josephson, comme par exemple les magnétomètres SOUID, les étalons de voltage, et les transformateurs alternatif-continu. Ces conceptions possèdent toutes la caractéristique essentielle que de très bas niveaux d'interférence magnétique, électrique et mécanique sont potentiellement réalisables.

used for cold cylinders, pistons, and displacers.

An experimental investigation of several concepts for very-low-power cryocoolers for operating highly-sensitive superconducting devices has been undertaken. The devices to be cooled are those using Josephson junctions. such as SQUID magnetometers, voltage standards and $A / D$ converters. The common basic feature of the concepts is that very low levels of magnetic, electric, and mechanical interference are potentially realizable.

Nylon and epoxy-glass composites have been

We have used unconventional materials and operating parameters in a classical type of heat pump to produce a new class of cryocoolers. They operate at low speed, with low input power, use mainly plastic and non-metallic composite materials for the cold parts, have only milliwatts refrigeration capacity, and are simple to build. Having such low refrigeration capacity, their initial cool down from room temperature requires many hours unless special measures are taken to speed up the process. The ultimate purpose of these cryocoolers is to cool small superconducting devises such as SQUIDS and Josephson voltage standards, whose cooling requirements are milliwatts to nanowatts or less.

The cryocoolers to be described here are Stirling machines, but the special features they incorporate could equally well be adapted to Gifford-McMahon and Viulluemier machines. One of the special features is the use of a plastic (nylon) and a composite Iglassreinforced epoxyl for the cold parts of the machine and also to provide regenerative heat exchange. In fact the regenerators consist of the annular gaps between the pistons and cylinders with the walls of each providing thermal storage. At an operating speed of the order of $1 \mathrm{~Hz}$, heat penetration into

The authors are with the Electromagnetic Technology Division. National Bureau of Standards, Boulder, Colorado, 80303. This paper was presented to Commission A1/2 at the XV International Congress of Refrigeration in Venice, Italy. This work was supported by the US Naval Air Systems Command
A multi-stage Stirling machine of these materials reliably maintains a temperature below $10 \mathrm{~K}$, with a few $\mathrm{mW}$ heat load and an input drive power of $50 \mathrm{~W}$. A single-stage machine maintains a temperature in the neighbourhood of $4 K$ with the hot end at $10 K$ and a drive power of the order of $5 W$. Specific heats and thermal conductivities of the materials have been measured between 5 and $10 \mathrm{~K}$, for purposes of analysis of regeneration inefficiency.

these materials is the order of $2 \mathrm{~mm}$ at $4 \mathrm{~K}$ so that regenerative heat exchange is limited by a thermal resistance in the material rather than in the working fluid, as is usually the case for regenerators made of $\mathrm{Pb}$ shot or other highly-conducting materials. The operation of these machines has been analyzed in detail by Radebaugh.'

One cryocooler described below is a multi-stage machine with nylon displacer and glass-epoxy cylinder which maintains $8.5 \mathrm{~K}$ or less with no heat load. The second machine is a single-stage two-piston arrangement which maintains 3 to $4 \mathrm{~K}$, with the hot end at 8 to $12 \mathrm{~K}$. This machine operates in the twophase region. That is, it liquefies helium at the cold end, so the cold-end temperature is the boiling point of helium at the average operating pressure, and the lowest temperature was achieved with an average pressure considerably less than atmospheric.

Since both of these machines have been described in the literature, ${ }^{2-4}$ their essential features will be only briefly summarized here. Following this we will speculate on their limitations and further developments, particularly on the possibility of combining them into a single unit capable of maintaining temperatures below $4 \mathrm{~K}$ in a $300 \mathrm{~K}$ environment.

\section{Four-stage stirling refrigerator}

A schematic of the four-stage machine ${ }^{2}$ is shown 
in Fig. 1. At an operating speed of $1 \mathrm{~Hz}$ and an average pressure of $0.5 \mathrm{MPa}$ the SQUID attached to the cold end was operated at $8.5 \mathrm{~K}$. A brass gasthermometer bulb with an internal volume of $0.5 \mathrm{~cm}^{3}$ was interposed between the SQUID and the bottom end of the displacer cylinder. The bulb was connected to a pressure gauge at room temperature through a $0.8 \mathrm{~mm}$ inside diameter $\mathrm{Cu}-\mathrm{Ni}$ tube, and the system was filled with helium gas at a pressure of $0.06 \mathrm{MPa}$ with the bulb at $8.5 \mathrm{~K}$. The thermal resistance and heat capacity of the thermometer bulb were intended to smooth out the amplitude of the temperature oscillations of the SOUID, relative to the amplitude at the displacer cylinder itself. The amplitude as indicated by the pressure gauge was about $0.05 \mathrm{~K}$, peak-to-peak.

Although Nb point contacts and SQUIDS can be operated at 8 to $9 \mathrm{~K}$, their performance and that of several other devices improve at lower temperatures. Probably the main limitation on the cold end temperature of this machine is the poor regenerator performance. The total regenerator heat capacity of the final stage operating between 8.5 and $20 \mathrm{~K}$ is estimated to be $0.1 \mathrm{JK}^{-1}$ compared to $.02 \mathrm{JK}^{-1}$ for the working fluid. Approximate average parameters used for this estimate are thermal conductivity, $x=1.5 \mathrm{~mW} \mathrm{~cm}^{-1} \mathrm{~K}^{-1}$; specific heat, $C$ $=50 \mathrm{~m} \mathrm{Jm}^{-3} \mathrm{~K}^{-1}$; radius, $\mathrm{e}=2.2 \mathrm{~mm}$; length, $\mathrm{l}=$ $15 \mathrm{~cm}$, and operating speed, $\omega=2 \pi$ radians $\mathrm{s}^{-1}$ : from which the total heat capacity is $\sim 2 \pi \rho / \sqrt{2} x \mathrm{C} / \omega$. The working fluid heat capacity is simply the specific heat of helium at the peak pressure, estimated to be $0.7 \mathrm{MPa}$ at a cold end temperature of $8.5 \mathrm{~K}$, multiplied by $\pi \rho^{2} d$, where $d=7 \mathrm{~mm}$ is the displacer

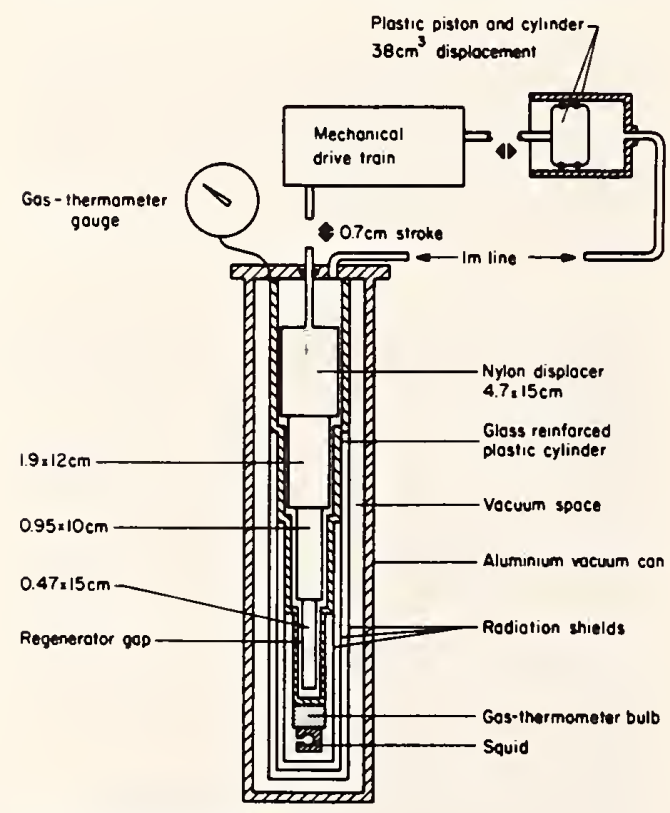

Fig 14 stage stirling refrıgerator

Fig. 1 Cryoréfrigérateur à cycle Stirling à 4 étages stroke. A heat capacity ratio of 5 is too small to provide efficient regenerative heat exchange, and the ratio becomes rapidly smaller if a lower cold-end temperature is assumed. A simple way to increase the ratio, using the same materials, would be to provide an additional annular gap or gaps for additional regenerator area, as was done in the single-stage machine described in the following paragraph.

\section{Single stage twin piston stirling refrigerator}

Another way to improve the lower stage performance is to reduce the helium pressure so as to enhance the ratıo of the regenerator-to-helium heat capacity. This will also reduce the available cooling power, but for small heat loads the temperature achieved can be lower. This approach did not work for the multistage machine because the larger refrigeration power at higher pressure was essential for the upper stages. However, a single-stage low-pressure machine could be operated as a separate additional stage at the bottom of the higher pressure multistage device. To test this idea, we built and tested a single-stage twinpiston Stirling machine which produces several $\mathrm{mW}$ of refrigeration in the 3 to $4 \mathrm{~K}$ range with upper end temperatures of 8 to $12 \mathrm{~K}$

Fig. 2 is a cross sectional drawing of the device. The two pistons operate concentrically inside a fixed cylinder and the movement of helium gas between the bottom end and the room temperature housing is limited only by the small radial clearances between the three concentric pieces. All three pieces were made from commercially available spun-glass epoxy $\left(' \mathrm{G}-10^{\prime}\right)$. At room temperature the viscosity of helium is large and thus little gas moves between the upper housing and the cold parts during each cycle of the machine (the speed is $1 \mathrm{~Hz}$ ). On the other hand, the reduced viscosity at low temperature results in large flow and thus little pressure drop between the compression and expansion spaces. As shown in the figure, this machine has two regenerator gaps in parallel to further enhance the heat-capacity ratio.

An electric heater was mounted on the hot end flange which was in turn connected through a weak thermal link to a liquid helium bath. With this arrangement the hot end could be maintained in the temperature range of 8 to $12 \mathrm{~K}$. A second heater was mounted on the cold end to determine refrigeration capacity. Carbon resistance thermometers were used to monitor the temperatures at the cold and hot ends as well as at several intermediate points. Changes in the helium boil-off rate were used to determine the magnitude of the heat rejected at the hot end.

For most of our experiments the temperature of the cold end was limited by entry into the two-phase region. The presence of liquid (of order $0.01 \mathrm{~cm}^{3}$ ) was confirmed by observation of the warming rate of the cold end after the refrigerator was stopped. The cold end temperature would typically remain at that of the liquid phase for several minutes and then rise quickly after the last of the liquid evaporated. With this confirmation of two-phase operation, it was clear that the operating temperature was essentially the 
equilibrium vapour pressure of the helium gas which was measured with the gauge shown in Fig. 2. A temperature of $3.1 \mathrm{~K}$ was achieved at an average pressure of $0.027 \mathrm{MPa}(200 \mathrm{~mm} \mathrm{Hg})$ with the hot end at $8.6 \mathrm{~K}$. With the hot end at $12 \mathrm{~K}$ and a pressure of $0.08 \mathrm{MPa}(600 \mathrm{~mm} \mathrm{Hg})$, the cold end reached a temperature of $4 \mathrm{~K}$. The heat ejected at the upper end (for this latter experiment) was about $50 \mathrm{~mW}$ and the refrigeration power at $4 \mathrm{~K}$ was $4 \mathrm{~mW}$.

We have measured the heat capacity and thermal conductivity (these will be reported elsewhere) of the spun-glass epoxy regenerator material and it is instructive to see how these influence the regenerator performance. For the following example we take the operation from 12 to $4 \mathrm{~K}$ as representative of the cooler operation. The diffusivities, $D=x / C$, at 4 and $12 \mathrm{~K}$, respectively, are 0.36 and $0.063 \mathrm{~cm}^{-1} \mathrm{~s}^{2}$. For $1 \mathrm{~Hz}$ operation the thermal penetration depth is $3.3 \mathrm{~mm}$ at the cold end and $1.2 \mathrm{~mm}$ at the hot end. The bottom end value indicates that a substantial portion of the available material is indeed used for heat storage at the lower end. The heat capacity of the entire regenerator surface (including the gaps inside and outside the lower stationary insert) is approximately $0.17 \mathrm{JK}^{-1}$. The average value of gas heat capacity at $0.08 \mathrm{MPa}(600 \mathrm{~mm} \mathrm{Hg})$, is $0.0087 \mathrm{JK}^{-1}$ so that the ratio of regenerator capacity to heat capacity of the gas is $\sim 20$. For comparison, the ratio would be only about 2 if the operating pressure were near $1 \mathrm{MPa}$ las in the multistage device). The need for reduced operating pressure is thus obvious.

P. Roubeau (private communication) has pointed out that gravity separation of the phases may play an important role in the operation of this machine. Being relarively incompressible, the liquid which collects in the bottom of the expansion space no longer takes part directly in the refrigeration cycle, but by filling the dead space and providing thermal ballast it may significantly increase the efficiency of the process. This is not completely certain, since the non-ideal characteristics of the fluid could offset this gain'. Obviously this action would depend on the attitude of the machine relative to gravity.

\section{Future developments}

For many purposes, an improvement of the multistage refrigerator (as discussed earlier) to achieve a temperature of 6 or $7 \mathrm{~K}$ will be adequate. However, to reach 3 or $4 \mathrm{~K}$ will require a combination of the two machines. The overall size and power requirement of the combination will be smaller if the low pressure stage is reduced in size and if it operates over a narrower temperature difference. If the diameter were reduced by a factor of two, then the volumes and heat ejected at the warm end would be four times smaller. The reduced cooling capacity could be compensated in part by an improvement in the performance of the multistage machine. As discussed earlier, this would require an increase in the overall heat capacity of the regenerator material, particularly in the bottom stage. It is thus conceivable that the combination might be comparable in size and power requirement to the multistage machine described above.

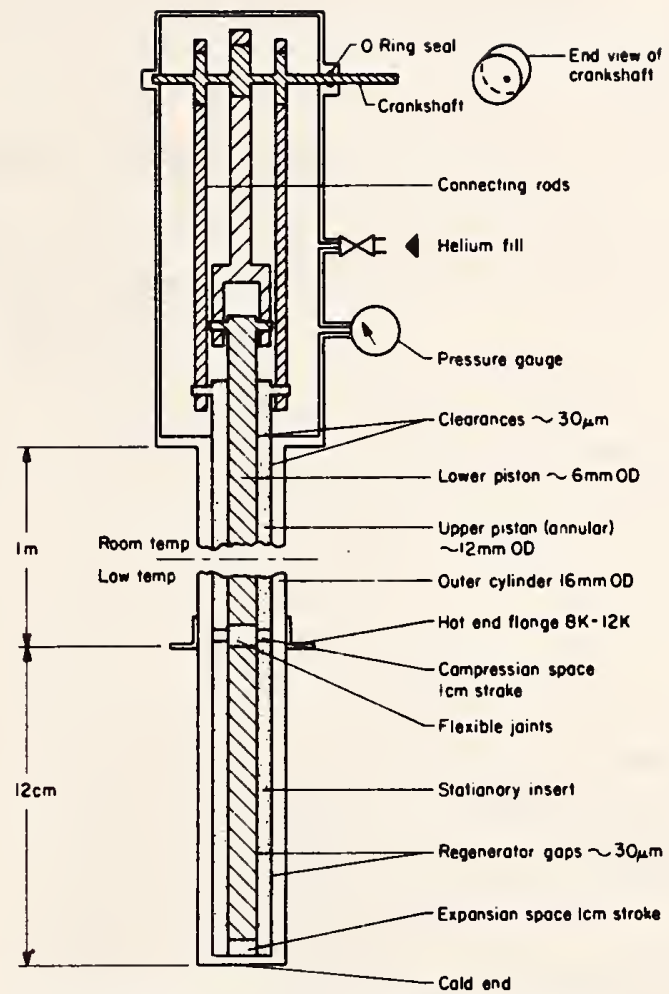

Fig. 2 Single stage iwin piston stirling refrigerator. The flexible joints permit self-centring of a centre piston so as to reduce frictional heating

Fig. 2 Cryoréfrigérateur à cycle Stirling à double piston à un érage. Les joints flexibles permettent un auto-centrage du piston central afin de réduire l'échauffement par frottement

With regard to the geometry of the combination, the two could be operated side by side with the hot flange of the single-stage coooler connected to the cold end of the other machine. The two would obviously operate from a single drive motor. However, it might be advantageous to consider a concentric configuration with the single-stage machine within the multistage one. Besides simpler machine work the heat conduction in the single-stage device would naturally be intercepted by higher temperature stages in the multistage machine. Also, the regenerator area of the multistage machine (particularly at the lower end) would be increased because of the larger diameter necessary to sccommodate the coaxial single-stage machine.

\section{References}

1 Radebaugh, R. Analysis of regenerator inefficiency for Stirling. cycle refrigerators with plastic displacers, Proceedings of the XV International World Congress of Refrigeration (1979)

2 Zimmerman, J. E., Radebaugh, R. Operation of SQUID in a very low-power cryocooler, NBS Special Publication 508 (1978) 3 Zimmerman, J.E., Radebaugh, R., Siegwarth, J.D. Possible cryocoolers for SQUID magnetometers, Superconducting Quantum Interference Devices and Their Applications (H. D. Hahlbohm and H. Hübbig, eds) Berlin. Walter de Gruyter I1977) 287

4 Zimmermen, J. E., Sullivan, D. B., A milıwatı Stirling cryocooler for temperatures below 4 K Cryogenics (March 1979) 
Proceedings of the SQUID Geophysics Workshop, Los Alamos, NM (June 1980).

CRYOCOOLERS FOR GEOPHYSICAL SQUID MAGNETOMETERS ${ }^{\dagger}$

James E. Zimmerman

National Bureau of Standards

El ectromagnetic Technology Division

Boulder, CO 80303

\section{ABSTRACT}

No alternatives to liquid-helium cryostats for SQUID geomagnetic measurements are presently available, but micro-miniature Joule-Thomson and lowpower non-magnetic stirling cryocoolers are being developed for this and similar purposes. With increasing interest and experimental work on the subject during the past year or two, it is likely that demonstrations of feasibility will occur in the moderately near future, and perhaps even a suitable commercial cryocooler in the next few years.

\section{TEXT}

To design a small closed-cycle cryocooler for use with geophysical SQUID magnetometers will be much more difficult than for a gradiometer, since the latter is insensitive to motion (in particular, rotation) in the earth's field caused by the cryocooler. Clearly, the problem is not impossible. That is, a system might consist of a small helium liquefier, probably operated intermittentiy, to maintain liquid helium in a magnetometer dewar, the vapor being collected for recycling. A systen of this type has been operated in the laboratory (Vincent, 1978). While this type of system clearly is not what most of us have in mind when thinking of a cryocooler for a SQUID magnetometer, perhaps it should not be rejected out of hand. A fraction of a liter volume is sufficient for a 3-axis magnetometer, and by efficient design a dewar of this size could operate at least 24 hours on one filling. A liquefier operating 10 hours per day should therefore have a capacity of the order of $0.05 \mathrm{~L} / \mathrm{hr}$. This would require $6 \mathrm{~W}$ of mechanical input power for a perfect liquefier, or $120 \mathrm{~W}$ if one assumes an efficiency of $5 \%$. A gas holder for the gas equivalent of 1/2 liter of liquid would be somewhat bulky, about $1 / 2 \mathrm{~m}^{3}$ at $2000 \mathrm{~m}$ above sea level.
With regard to efficiency, a statistical survey (Strobridge, 1974) several years ago suggested that efficiency of helium liquefiers and refrigerators drops dramatically at refrigeration capacities less than a watt or so at $4 \mathrm{~K}$. Perhaps this trend should not be taken too seriously, however, wt thout more specific studies on the itmitations of small, low-power cryocoolers. A SQUID cryocoeler butit in NBS-Boulder (see below) has an efftciency of about $1 \%$ ( $8 \mathrm{~mW}$ refrigeration at $8 \mathrm{~K}$ with an estimated electrical power input of $30 \mathrm{~W}$ ), which is still comparable to the efficiences of cryocoolers 10 to 100 times larger, at the lower end of the statistical survey.

The total heat leak, and the size of the system, might be reduced significantly by incorporating the I quefier into the magnetometer dewar itself. Also, the dewar capacity can be reduced if the liquefier is operated a few minutes out of each hour rather than a few hours out of each day, as suggested above. Although no systems of this type have been built, it is probably the one most commonly envisioned as a compromtse between the inconvenience of the conventtonal cryostat requiring periodic transfer and filling from a (usualiy distant and expensivel source of liquid helium, and the difficulty of designing a continuously running but noninterfering cryocooler. It is obviously limited in application to short-term intermittent operations, and does not eastly lend itself to operation in arbitrary orientations.

The tdeal system, from the point of view of efficiency, portabllity, and flexibility is a continuousiy runnting cryocooler. Currently, two cryocooler types with some potential of being adaptable to geophysical SQUID magnetometers are being developed.

tContribution of the U. S. Government, not subject to copyright. 
One of these is the micro-miniature JouleThomson cooler proposed by Little and subsequently investigated experimentally using silicon chip microcircuit technology to form the channels for the counter flow heat exchanger and the JouleThomson throttle (Little, 1978 a and b). The high thermal-conductivity of sflicon makes it impractical for very low-temperature refrigeration, and recently attention has been directed toward adapting the idea and techniques to low-conductivfty materials such as glass. A practical cryocooler based on Little's idea has not yet been reported, so we will speculate on some of its potential advantages and dtsadvantages. First, and maybe foremost, there are no solid moving parts at low temperature, and it was pointed out by Little that fluid flow in the narrow $(\sim 10 \mu \mathrm{m})$ channels of a micromintature Joule-Thomson cooler should be laminar. Consequently, there should be no high or low-frequency noise generated by this part of the system - a matter of cructal importance for SQUIO magnetometers. Second, the essential low-temperature elements of a Joule-Thomson cooler, the counter flow heat exchanger and the throttle section, can be made of very narrow channels which might be buflt into the SQUIO itself and its electrical leads, so that the system cools precisely the part that needs to be cooled and nothing else. This is the essence of Little's idea, to develop a flexible technique for matching the cryocooler to the requirement of a spectfic device. Third, owing to the inherent low heat capacity of the system, cool-down time could be of the order of 1 minute. This would be ideal for applications like geothermal prospecting where measurements are intermittent and shortterm.

On the other hand, the narrow channels of the Joule-Thomson system may be easily clogged with impurfties (frozen afr, grease, dust particles, etc.) which may be difficult to remove. Second, at least three separate stages $\left(\mathrm{N}_{2}, \mathrm{H}_{2}\right.$, and $\mathrm{He}$, for example) are required to reach the operating temperature of presently available SQUIDs, and rather high pressures are required for the higher temperature stages. For many applications, gas from high-pressure cylinders could be used, and Little has estimated in a particular example an operating time of 400 hours from a standard cylinder of gas. Third, a closed system for continuous long-term operation would require three compressors. These could be remotely located to avold interference, but the relatively high pressures and stringent limits on solid or condensable impurities would tend to make the system rather complicated and expensive. Fourth, the Joule-Thomson effect is thermodynamically ineffictent. However, the ineffictency might be more than compensated for by optimally matching the cryocooler to the SQUID as suggested above.

A quite different SQUIO cryocooler is being tested in the NBS-Boulder Laboratories (Zimmerman, et al., 1980, 1978a, 1978b, 1976, Sullivan and Zimmerman, 1979). This is a standard Stirling cryocooler ${ }^{*}$, with some untque features to adapt it for use with SQUIDs, operating in the nefghborhood of $7 \mathrm{~K}$. In its present version, shown schematically in Fig. 1, a soltd nylon stepped displacer moves up and down in a matching epoxy-glass compostte cylinder. This type of cryocooler is simple, easy to build, eastly portable, operates on rather low input power, and is potentialiy capable of long-term continuous unattended operation. An earlier version operated a total of more than 7000 hours (not continuousiy) without observable wear or degradation of performance.

The problem with this machine, which overshadows all others, is that it directly and indirectly generates magnetic interference. Tests made with a transverse SQUIO magnetometer, mounted on the cold end of an earlier version of the machine, showed that a peak-to-peak signal of about $0.5 \mathrm{nT}$ was generated by rotation of the SQUID in the earth's field due to flexing of the displacer cylinder at the cryocooler operating frequency of $1 \mathrm{~Hz}$. There were also unidentiffed higher frequency components with an amplitude of the order of $10 \mathrm{pT}$. With the present gradiometer-cryocooler, a rather clean $1 \mathrm{~Hz}$ interfering signal was measured with an amplitude corresponding to a peak-to-peak field of about $0.3 \mathrm{pT}$ in the gradiometer loop nearest the cold end of the displacer cylinder. Since the gradiometer may be assumed to be insensitive to rotation In the earth's ffeld, this interference is probably caused by the diamagnetic moment of the moving nylon displacer in the earth's ffeld, or by the changing paramagnettc moment of the glass-epoxy cylinder walls due to local temperature varfation during the refrigeration cycle. We have determined that the susceptibiltty of nylon is about the same as that of quartz (about $10^{-5}$ ).

Although the interference components identified above are rather large, it should be appreciated that "clean" signals at the cryocooler frequency and multiples thereof can be largely eliminated by any of various methods of subtracting the interference from the magnetometer output. By this and other methods of interference rejection, it appears that the system can be made practical for some of the less-demanding applications in geophysics, perhaps at the picotesla level of sensitivity.

It would be dffficult to guess when practical cryocoolers wtll be generally avatlable for geomagnetic measurements. In the past two or three years several other laboratories have started experiments on both microminiature Joule-Thomson coolers and on plastic Stirling cryocoolers similar to the above, so it is concelvable that something may be commercially avatlable within a few years.

*The sttrling machine, whtch is probably the stmplest and most effictent small heat engine for large temperature differences, was invented in 1816 and used to pump water out of a mine. The first stirling refrigerator was butlt about 1860 . It was Aighly successful (Walker, 1973). 
Work on the Stirling cryocoolers is being done in collaboration with D. B. Sullivan, and is supported in part by the Office of Naval Research and the Naval Coastal Systems Center.

\section{REFERENCES}

Little, H. A., 1978, Design and Construction of Microminiature Cryogenic Refrigerators, Future Trends in Superconductive El ectronics, edited by B. S. Deaver, Jr., et al., AIP Conference Proceedings No. 44, p. 421 .

Little, W. A., 1978, Scaling of Minlature Cryocoolers to Microminiature Size, NBS Special Publication 508, Apri1 1978, p. 75.

Sullivan, D. B. and Zimmerman, J. E., 1979, Very lowpower Stirling Cryocoolers using plastic and composite materials, International Journal of Refrigeration, v. 2. p. 211-213.

Strobridge, T. R., 1974, NBS Tech. Note 655.

Vincent, D. A., 1978, Closed-Cycle Refrigerator for a Superconducting Susceptometer, NBS Special Publication 508, April 1978,p. 131.

Walker, G., 1973, Stirling-Cycle Machines, Oxford Press.

Zimmerman, J. E. and Radebaugh, R., 1978, Operation of a SQUID in a Very Low-Power Cryocooler, NBS Special Publication 508, Aprtl 1978, P. 59.

Zimmerman, J. E., et al., 1976, Possible Cryocoolers for SQUID Magnetometers, Superconducting Quantum Interference Devices and their Applications, editors, H. O. Hahlbohm and H. Lübbig, Walter de Gruyter.

Zimmerman, J. E., 1978, Crycoolers for Superconductive Electronics, Future Trends in Superconductive Electronics, edited by 0.5 . Deaver, Jr., et al., AIP Conference Proceedings No. 44, p. 287 .

Zimmerman, J. E. 1980, Cryogenics for SQUIDs, Presented at the Second International Conference on SQUIDs, Berlin, May 6-9, 1980. To be published. Preprints avallable.

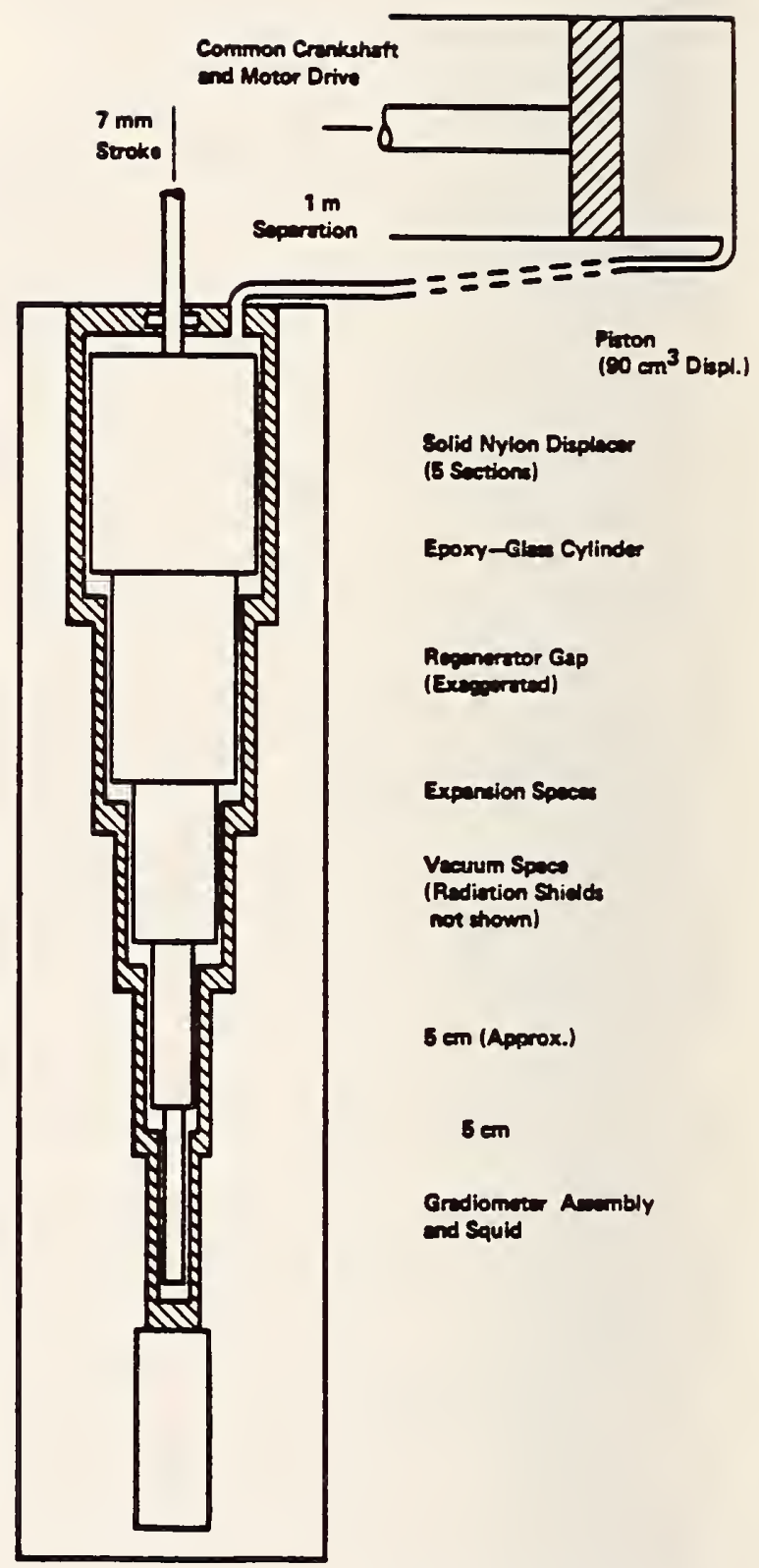

Figure 1. - Simplifted schemattc crosssection of SQUID cryocooler, approximately to scale. Note different hortzontal and vertical scales. Nonmagnetic crankshaft and crosshead and magnetically shielded $50 \mathrm{Ndrive}$ motor not shown. Total weight about $20 \mathrm{~kg}$. (See Zimerman, 1980 for detailed spectfications and performancel. 
"SQUID 80: Superconducting Quantum Devices and their Applications," (H. D. Hahlbohm and H. Lübbig, eds.), Berlin, walter de Gruyter (1980), pps. 423-443.

CRYOGENICS FOR SQUIDS

James E. Zimmerman

Electromagnetic Technology Division

National Bureau of Standards

Boulder, Colorado 80303 U. S. A.

\section{Abstract}

Liquid-helium cryostats continue to be the principal mechanism for cooling SQUIDs and SQUID instruments, but there is increasing interest in self-contained closedcycle cryocoolers as an alternative. Mechanical gas refrigerators, in various configurations, and the JouleThomson effect seem likely to be the most practical mechanisms, but magnetic, electric, and other processes may be useful if suitable materials can be developed. While not much work on SQUID cryocoolers as such has been done yet, a large amount of work on higher temperature refrigerators for infrared sensors and for use in spacecraft provides a sophisticated technical base upon which to build. A low-power five-stage stirling, cryocooler with a single-component SQUID gradiometer has begun operation in the author's laboratory. Although this machine requires only $20 \mathrm{~W}$ of mechanical power input to maintain a temperature of $7 \mathrm{~K}$, a large reduction of input power is theoretically possible.

\section{Introduction}

Liquid-helium cryostats continue to be universally used to cool SQUIDs and other superconducting devices. During the past year or two, however, there has been increasing interest, both in government funding agencies and in private industry, for the 
development of small cryocoolers for SQUID magnetometers and similar instruments, and of somewhat larger machines for superconducting computers (1), (2).

For many years there has been extensive development of small refrigerators for infrared detectors at higher temperatures, above $20 \mathrm{~K},(3)$ and for masers (2). Much of this technology and experience will be directly useful in the development of cryocoolers for SQUIDs. Unfortunately, much of the literature is in the form of government reports, not readily available, even though unclassified.

A short but imaginative and comprehensive review of refrigeration principles and practice has been written by Radebaugh (4). A very comprehensive treatise on mechanical gas refrigerators called "Cryocoolers" is in preparation by G. Walker for publication within a year by Plenum press. It contains more then 1000 references. The various proceedings of the Intersociety Energy Conversion Engineering Conference are a rich source of information and ideas on all kinds of heat engines.

A recent review on cryogenics for spacecraft, including cryocoolers, solid and liquid cryogens, and passive radiation cooling, has been written by Sherman (5). The extreme reliability and other special requirements for cryogenics in space has resulted in improvements in bearing and seal design, materials, portability, and efficiency, which will be useful in the design of cryocoolers for SQUIDs.

\section{A. MACHINES AND PRINCIPLES}

Most work in the U. S. A. and elsewhere on small cryocoolers, below $20 \mathrm{~K}$, and on higher temperature machines has been on stirling, Vuilleumier, and Gifford-McMahon machines, shown schematically in Fig. 1. All of these use displacers and regenerators. The regenerator may be built into the displacer, 

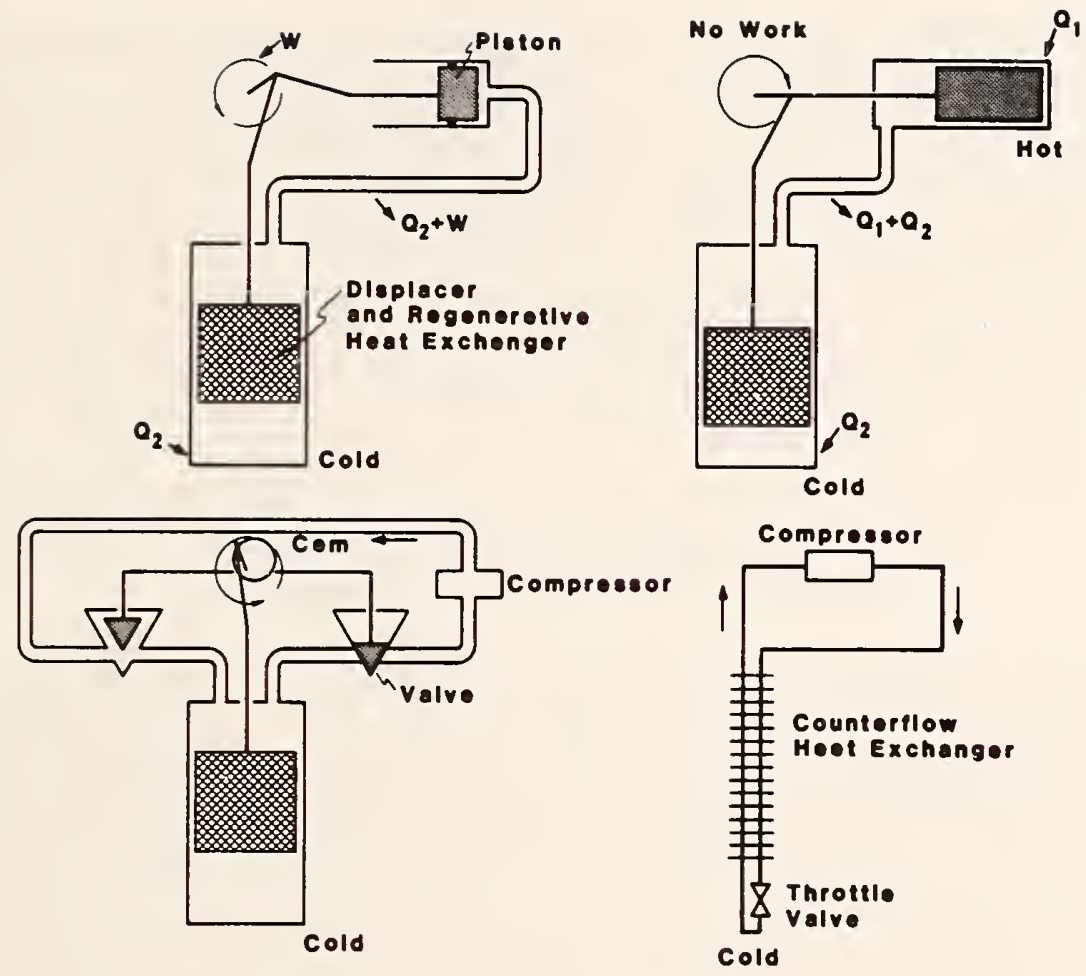

Fig. 1. Schematic representation of Stirling (upper left), Vuilleumier (upper right), Gifford-McMahon (lower left) and Joule-Thomson refrigeration systems.

as shown, or into the walls of the surrounding cylinder, or as a separate unit. In the simplest machines (see, for example, Appendix A), regenerative heat exchange takes place between the surfaces of the displacer and of the cylinder and the working fluid in the intervening gap. In the stirling machine the displacer and piston are driven in synchronism, with a phase difference of the order of $\pi / 2$, and likewise for the two displacers of the Vuilleumier machine. Both the Stirling and the Vuilleumier are reversible in the sense that they can pump heat either direction, and a stirling machine can be used with a drive motor as a refrigerator or as an engine delivering work to an external load. In a 
Gifford-McMahon machine the compressor operates independently of the displacer, and the pressure in the displacer cylinder is raised and lowered through valves appropriately synchronized with the motion of the displacer. This type of machine is not simply reversible, and is probably less efficient than the reversible types. It has a significant advantage, for SQUID users, that the potentially noisy compressor can be remotely located. All of these types of machines have achieved temperatures in the neighborhood of 6 to $7 \mathrm{~K}$, using multi-stage or stepped displacers. For temperatures down to $4 \mathrm{~K}$, a Joule-Thomson final stage can be added. An alternative to a Joule-Thomson stage has been proposed (6).

There are many other recognized cooling machines (Solvay, Claude, etc.), mainly using helium gas, or sometimes hydrogen, as the working fluid. Then there is a spectrum of mechanisms using magnetic, or perhaps electric, polarizable material as the working fluid, and machines operating in the neighborhood of critical temperature of any of various fluids. "Evaporative" coolers such as home refrigerators and helium liquifiers using the Joule-Thompson effect in the final stage are examples of the latter.

Magnetic cooling techniques (an extension of classical methods of achieving temperatures below $1 \mathrm{~K}$ ) have been under study for several years both at higher temperatures, and, more recently, in the range of 2 to $20 \mathrm{~K}(7,8,9)$. These might be difficult to use with SQUID magnetometers, however, because of interference. Paraelectric cooling of SQUIDs is an interesting possibility, but suitable materials have not yet been found (10).

Refrigeration is a difficult subject to deal with summarily. Thermodynamics provide a set of simple and elegant general principles on the minimal conditions necessary to achieve refrigeration and on the maximum refrigeration rate that can 
be achieved under specific conditions, but offers no clue as to a possible mechanism to achieve that rate. For example, a source of heat $\dot{q}$ at an elevated temperature $T_{1}$, such as the sun or a piece of coal, can be used to remove heat, that is, to refrigerate, at a rate $\dot{Q}_{2}$ from a region at a low temperature $\mathrm{T}_{2}$, where both $\dot{Q}_{1}$ and $\dot{Q}_{2}$ are ultimately rejected to the environment at temperature $T_{0}$. The quantitative relation for the maximum possible refrigeration rate is

$$
\dot{Q}_{2}=\dot{Q}_{1} \frac{T_{2}}{T_{1}} \frac{T_{1}-T_{0}}{T_{0}-T_{2}}
$$

An example of a refrigerating mechanism is a thermoelectric generator connected to a Peltier cooler - two thermocouples in parallel with the connecting wires maintained at ambient temperature. With conduction electrons as the only moving parts, such a mechanism should be highly reliable. Suitable materials for practical cryocoolers using this mechanism do not seem to have been discovered, however. A more practical example is the vuilleumier machine (11). It has moving parts, but no work is done on or by them, so mechanical forces are small. Such machines are completely self-contained in the sense that they can be operated with "primary" energy sources such as the sun or petroleum-derived fuels. Thus, they may be attractive for use in space and other remote locations.

When a source of mechanical work, such as an electric motor, is available, then the maximum possible refrigeration $\dot{Q}_{2}$ is related to the mechanical power $W$ by

$$
\dot{\mathrm{Q}}_{2}=\mathrm{W} \frac{\mathrm{T}_{2}}{\mathrm{~T}_{\mathrm{o}}-\mathrm{T}_{2}},
$$


where $\mathrm{T}_{2}$ and $\mathrm{T}_{0}$ are as defined above. Most of the betterknown refrigerating machines, Stirling, Gifford-McMahon, and so on, are of this class. They are of interest to the majority of those who are located near "secondary" energy sources such as electric power lines.

Two commonly used idealizations of thermodynamic cycles achieved by the above machines are the stirling cycle and the Ericsson cycle. The former consists of an isothermal compression in the warm (room-temperature) end of the machine, a constant volume displacement of gas from the warm end to the cold end through the regenerator, an isothermal expansion, and a constant volume displacement back to the warm end. The Ericsson cycle consists of the same two isothermal processes, but the processes of displacement are done at constant pressure rather than constant volume. A Stirling machine can approximate the stirling cycle if it is operated slowly with a phase difference of $\pi / 2$ between piston and displacer motions. It can equally well approximate the Ericsson cycle, if the phase difference is shifted so the compression process partly overlaps the downward displacement (from warm to cold ends) of the working fluid and the expansion process overlaps the upward displasement.

\section{B. Interference With SQUID Operation}

Cryocooler parameters of interest to users of SQUIDs are cost, reliability, ease of use and operation, cooldown time, refrigeration rate, power input, size and weight, temperature stability. Above all, users of SQUIDs will want to know whether cryocoolers can be developed which will not interfere significantly with their operation. SQUID magnetometers are being used in geophysical applications where very low frequency fields, below $1 \mathrm{~Hz}$ are measured to $1 \mathrm{pT}$ or better. Gradiometers used for geomagnetic anomaly detection at frequencies below $1 \mathrm{~Hz}$ and for biomagnetism between one and $100 \mathrm{~Hz}$ require 
noise and interference levels at the gradiometer pickup loops of 0.1 to $0.01 \mathrm{pT}$ or less.

Operation of a SQUID in a cryocooler has been reported once previously. An interference level of $0.5 \mathrm{nT}$ at the $1 \mathrm{~Hz}$ operating speed was measured (12). It was shown that most of this was due to motion of the cold end of the cryocooler, on which the SQUID was mounted, in the earth's field.

Although the problem of reducing the interference level by several orders of magnitude is formidable, there are several ways of dealing with it, none of which have been exploited up to now. First of all, some careful attention to the cryocooler structure might result in at least an order of magnitude reduction in motion of the cold end. For SQUID magnetometers used in low dc fields, as in interplanetary space or in the shielded rooms of Massachusetts Institute of Technology, Physicalisch-Technische Bundesanstalt, and Helsinki Technical University, motional interference would be reduced by a factor of 1000 .

For a well-balanced gradiometer, motional interference in a uniform field would be reduced by up to $10^{6}$, and it should be possible to reduce direct magnetic pickup from the cryocooler by factors of 10 to 100 relative to that picked up by a magnetometer.

Since most interference, by whatever mechanism, from a cryocooler should be at the cryocooler frequency and its harmonics, it can in principle be largely eliminated from the SQUID output by a "commutative" filter synchronized with the cryocooler frequency. These filters are used very effectively in several laboratories to eliminate power-line interference from the output of biomagnetic SQUID gradiometers. If the magnitude of the interfering signal is essentially constant, the filter bandwidth can be made extremely narrow, so that 
virtually no information is lost from a typical signal. Since commutative filters may be unfamiliar to many, a primitive version of one is given here (Fig. 2). The rotating switch or commutator is rotated in synchronism with the interfering signal at $\omega_{0}$ that is, at the cryocooler speed. In modern practice, of course, the switching is done with solid-state integrated circuits (13). The rejection bandwidth of the filters at $\omega_{0}$ and its harmonics is $\Delta \omega=1 / R C$.

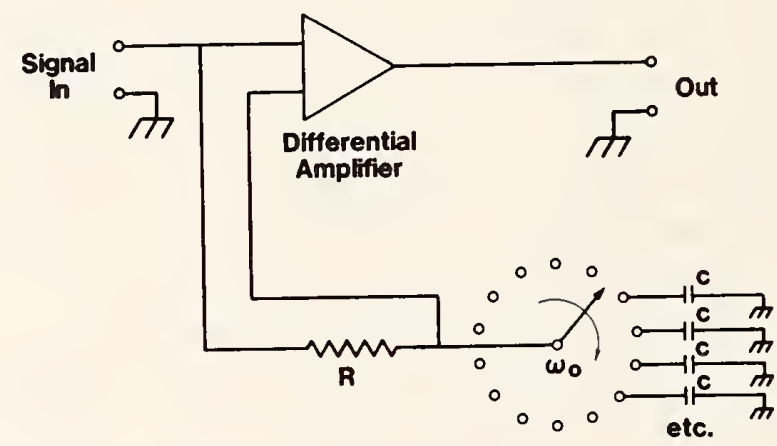

Fig. 2. Schematic of primitive version of a commutative filter for eliminating interference at $\omega_{0}$ and its harmonics.

C. Reliability

One of the most commonly expressed concern about cryocoolers is reliability. In private discussions, knowledgeable individuals have often stated that cryocoolers are not reliable. Some of these statements seem to be based on military-sponsored projects which have fallen short of the goal of producing cryocoolers for several years of continuous unattended operation. Most of us do not require such extreme 
performance, and in any case, users of cryocoolers for ground-based maser preamplifiers have reported outstanding reliability(2). Users of SQUID magnetometers may know that the reliability of liquid-helium cryostats leaves much to be desired, especially outside the laboratory. In 1973, for example, Wallace Campbell and the author measured the geomagnetic field variations during the solar eclipse of 1973 in southern Chad, near the geographic center of Africa (14). More recently, some measurements were made on electric currents in the Alaska pipeline induced by ionospheric currents (15). In each case, only one of two storage containers of liquidhelium arrived at the specified destination. These and similar experiences have demonstrated the need for selfcontained cryocoolers of modest reliability.

\section{NBS Cryocooler Development}

The first paper on cryocoolers for SQUIDs was presented $31 / 2$ years ago here at this conference (16). Since then, we have demonstrated that a simple, potentially low-cost cryocooler can be built which will maintain temperatures in the range of 7 or $8 \mathrm{~K}$ in which Nb SQUIDs operate very well (17), (also see Appendix). We have also built an even simpler, singlestage unit which achieved temperatures in the 3 to $4 \mathrm{~K}$ range with the hot end in the neighborhood of $10 \mathrm{~K}(6,17)$. Refrigeration capacities of all our machines have been on the order of a few $\mathrm{mW}$ per Kelvin temperature rise of the cold end, with correspondingly larger cooling capacities at the higher-temperature stages. Emphasis in all our work has been on low power and low interference levels, for compatibility with SQUID magnetometers and small gradiometers. One result of this emphasis has been machines maintaining 7 or $8 \mathrm{k}$ with driving power levels very much lower than those required for preyious machines in this temperature range. Our machines require 50 to $100 \mathrm{~W}$ electrical power, using 
inefficient ac drive motors. With reasonably efficient dc motors and other refinements, it is probable that temperatures below $10 \mathrm{~K}$ could be achieved with 10 to $20 \mathrm{~W}$ of electrical power.

While it has been rewarding to demonstrate that superconducting and even liquid-helium temperatures can be reached with low-cost materials and simple mechanisms requiring no great skill to assemble, the discussion which follows indicates that large improvements in efficiency may still be possible.

\section{E. Efficiency}

Efficiency is not usually of prime concern in the design of small cryocoolers. Still, it might be emphasized once more that the inherent refrigeration requirements for SQUIDs are smaller by orders of magnitude than that provided by most "small" or "miniature" cryocoolers.

Even though the cost of energy to run a cryocooler may not be significant (even these days), increasing the efficiency so as to reduce the size and power requirements of a SQUID cryocooler has potential benefits in terms of reduced capital cost, portability, and reduction of interference. In the following section are presented some numerical comparisons between liquid-helium cryostats, the NBS small cryocoolers, and the inherent refrigeration requirements for SQUIDs.

F. Liquid-Helium Cryostats, the NBS Cryocooler, and Inherent SQUID Requirements

A "super-insulated" helium dewar of 1 L capacity with vaporcooled radiation shields can have an evaporation rate of the order of $5.8 \times 10^{-3} \mathrm{~cm}^{3} / \mathrm{s}(1 / 2 \mathrm{~L} /$ day $)$. If we were to continuously replenish this liquid helium by the use of an ideal helium liquefier, the amount of mechanical power $W$ required would be 


$$
\dot{W}=\dot{m} \int_{4}^{300} c_{p} \frac{T_{0}-T}{T} d T+\left.\dot{m L} \frac{T_{0}-T}{T}\right|_{4}
$$

where $\dot{m}$ is the mass evaporation rate, $c_{p}$ is the specific heat at constant pressure, $\mathrm{T}_{0}=300 \mathrm{~K}$, and $\mathrm{L}$ is the latent heat of evaporation. For $1 / 2 \mathrm{~L} /$ day, the result is approximately $\mathrm{W}=5 \mathrm{~W}$. By comparison, the present and previous NBS cryocoolers have required 10 to $20 \mathrm{~W}$ mechanical power to maintain volumes considerably smaller than $1 \mathrm{~L}$ at temperatures of 7 or $8 \mathrm{k}$. We might conclude that these machines are reasonably efficient, but that there is room for significant improvement. There are at least four sources of inefficiency in the cryocoolers not present in a liquid-helium cryostat, namely, shuttle heat loss (due to cyclic mis-matching the temperature distributions along the displacer and cylinder surfaces), regenerator inefficiency (due to imperfect heat exchange between working fluid and regenerator), non-isothermal compression and expansion (also due to imperfect heat exchange), and mechanical friction.

On the other hand, liquid helium is a very non-ideal cryogen, because the refrigeration capacity is nearly uniformly distributed in temperature. For SQUID and other small device applications, the essential refrigeration requirements are heavily weighted toward the room-temperature end of the structure. An outer radiation shield at $150 \mathrm{~K}$, for example, should require more than 16 times as much refrigeration as one at $75 \mathrm{~K}$ receiving radiation from the outer shield at 150 $\mathrm{K}$. Similarly, thermal conductivity of most alloys and plastics increases with temperature. To demonstrate the effect of a more efficient distribution of cooling capacity, let us assume that the absorption of enthalpy by $1 / 2 \mathrm{~L} /$ day of evaporating liquid helium is proportional to the temperature of the structure (including electrical leads) at the point 
where absorption takes place. The total enthalpy change, from liquid helium to gas at $300 \mathrm{~K}$, is $6300 \mathrm{~J} / \mathrm{mol}$., which gives a total enthalpy absorption rate $\dot{H}=1.14 \mathrm{~W}$ at an evaporation rate of $1 / 2 \mathrm{~L} /$ day. The mechanical power $W$ required for an ideal refrigerator to provide the same distribution of refrigeration is

$$
W=\int_{4}^{300 K} \dot{Q} \frac{\dot{T}_{0}-T}{T} d T,
$$

where $\dot{Q}=A T$ and $A$ is chosen to satisfy the requirement $\dot{H}=\int_{4 \mathrm{~K}}^{300 \mathrm{~K}} \dot{\mathrm{Q}} \mathrm{dT}=1.14 \mathrm{~W}$. The result is $\mathrm{W}=1.13 \mathrm{w}$, more than four times smaller than the mechanical power required to produce $1 / 2$ L/day of liquid helium.

Our cryocoolers compare rather unfavorably with this more realistic idealization, especially when the relatively small cold volume of the cryocoolers is considered.

Most cryocoolers have been or are being designed for some specified "net". or "available" refrigeration at one or two specified temperatures, such as $40 \mathrm{~W}$ at $60 \mathrm{~K}$ and $1.5 \mathrm{~W}$ at 12 K.

For a SQUID cryocooler, it may be more economical not to specify any available refrigeration, but merely to require that the cryocooler maintain itself and the SQUID, with its bias and signal lead wires, at the operating temperature. As a final exercise on theoretical refrigeration requirements, let us consider the ideal mechanical power $w$ required to handle the heat conducted down the wires, assuming an electrical 
resistivity proportional to temperature, as might be the case for somewhat impure copper, with a room-temperature resistance for all the wires in parallel of $0.1 \mathrm{ohm}$. This corresponds, by the Wiedemann-Franz law, to a temperatureindependent thermal conductance $\mathrm{K}=7.3 \times 10^{-5} \mathrm{~W} / \mathrm{K}$. Consider two refrigeration systems which might be used to maintain opposite ends of the wires at $4 \mathrm{~K}$ and $300 \mathrm{~K}$ : (1) the worst possible, where all of the heat is pumped from $4 \mathrm{~K}$ to $300 \mathrm{~K}$, and (2) the best possible, where the refrigeration is optimally distributed along the length of the wires. In the first case

$$
W=\frac{T_{0}-T(0)}{T(0)} K\left(T_{0}-T(0)\right)=1.6 \mathrm{~W},
$$

where $T(0)=4 \mathrm{~K}$ and $\mathrm{T}_{\mathrm{O}}=300 \mathrm{~K}$, and the temperature distribution along the wires is linear. In the second case, $R$. L. Kautz and D. B. Sullivan have determined by variational calculus that the optimum temperature distribution is exponential.

$$
\frac{T(z)}{T(0)}=\left[T_{0} / T(0)\right]^{z} \text {, }
$$

where $z$ is the relative distance along the wires measured from the cold end. The ideal mechanical power is

$$
\begin{gathered}
W=\int_{0}^{1} \frac{T_{0}-T}{T} K \frac{d^{2} T}{d z^{2}} d z+\left.\frac{T_{0}-T}{T} K \frac{d T}{d z}\right|_{z=0} \\
=312 \mathrm{~mW}+93 \mathrm{~mW}=405 \mathrm{~mW} .
\end{gathered}
$$


The difference between the two cases is dramatic, especially considering that the operating resistance would be much lower in the second case, $0.023 \mathrm{ohm}$, than in the first case, $0.051 \mathrm{ohm}$, owing to the lower temperature distribution along the wires. The numerical values are derived from

$$
R=\int_{0}^{I} \frac{d R}{d T} \frac{d T}{d z} d z,
$$

$\mathrm{dR} / \mathrm{dT}$ being determined by the assumptions of a room-temperature value $R=0.1 \mathrm{ohm}$, and resistivity proportional to temperature.

A continuous distribution of refrigeration along a linear structure can be achieved in principle through the use of an elongated, variably tapered expansion space. An experiment with a long, conically tapered expansion space has been done by du Pre and Daniels (18).

The above discussion suggests a class of problems in optimal refrigeration designs, where conduction, radiation, and other heat inputs to the cold device, the wires, and to the refrigerator structure itself are dealt with as in the above example. Such problems are very complex, but the approach might lead to SQUID-cryocooler systems quite different from those contemplated so far. The work of $\mathrm{w}$. Little might be cited as an inspiration in this particular context.

\section{G. Microminiature Joule-Thomson Cooling}

Little has discussed the problem of scaling down the size of several different refrigeration systems by a factor of 100 or more (19). In particular, he has studied analytically and experimentally the possibility of making "microminiature" 
Joule-Thomson refrigerators by microcircuit techniques on silicon or other suitable substrates, to cool a thin-film device which might be part of a larger, room-temperature microcircuit on the same substrate (20). Silicon-chip technology has not been found suitable for cryogenic temperatures because of the large thermal conductance of the silicon, and present work is directed toward adapting the idea and techniques to other more suitable materials.

The fluid-flow channels in a microminiature Joule-Thomson cryocooler are so narrow, less than $0.1 \mathrm{~mm}$, that the counterflow heat exchanger might, in principle, be made to resemble a thin wire, with lower heat conductance than that assumed for the electrical wires in the numerical example above. Thus, the mechanical power necessary to handle the intrinsic heat leak of a Joule-Thomson cryocooler plus the refrigeration requirements of a SQUID together may be very small, in spite of the inherent inefficiency of the Joule-Thomson process.

It was also pointed out by Little that the fluid flow in the channels of a microminiature Joule-Thomson system should be laminar and so should generate no microphonic or other interference, a matter of some interest to SQUID users.

\section{H. Recent Deyelopments in Mechanical Gas Refrigerators}

The traditional drive mechanism for mechanical gas refrigerators is a crankshaft and connecting rods. However, some very interesting developments currently in progress are on the use of linear reciprocating magnetic drive motors directly connected to pistons and displacers, magnetic or gas bearings and suspensions, clearance seals, and mechanical resonance to reduce drive forces $(5,21,22,23,24,25)$. The term clearance seal refers to a piston and cylinder with sufficiently small radial clearance that leakage is negligible without 
liquid lubricants or piston rings. The piston can be centered in the cylinder by dynamic magnetic forces controlled by position sensors and servo loops, or by hydrodynamic or hydrostatic fluid forces. Either way, a piston can be centered surprisingly well in the cylinder, with radial clearnces of $25 \mu \mathrm{m}$ or less, so that there are no solid surfaces in contact, and theoretically, no wear. With such small clearances it is necessary to use materials with good dimensional stability, and also to use hard materials to avoid disaster in case some rubbing does occur. Possible materials include chromium oxide surfacing on aluminum, nitrided steel, certain metal carbides, and ceramics. (We have made a 50-mm diameter piston and cylinder of fine-grained aluminum oxide ceramic, with $4 \mu \mathrm{m}$ radial clearance, which have passed preliminary tests but have not yet been used as a compressor. For this purpose we will use the traditional crankshaft and crosshead to couple to a drive motor.) By coupling the piston and reciprocating drive motor to a metal, magnetic, or gas spring (the latter might be called a bounce chamber or some such term), a mechanical resonance can be made to match the desired drive frequency, so that the drive motor need only supply the forces inherent in the refrigeration process and various irreversible effects.

With regard to irreversibility, or lack thereof, G. M. Benson (private communication) has achieved excellent efficiency with small stirling engines and refrigerators, partly through the use of isothermalizers, that is, systems of concentric interleaving cylindrical rings in the expansion and compression spaces which provide large heat transfer areas so that so-called isothermal processes are actually very nearly isothermal (23). 
Appendix A

Figure 3 is a sketch of a stirling cryocooler with 5-stage displacer which maintains temperatures below $7 \mathrm{~K}$. Figure 4 summarizes the operating characteristics of the cryocooler. The temperature of the cold end is shown as a function of electrical heat input to an aluminum cylinder 13 by $130 \mathrm{~mm}$ which simulated the SQUID gradiometer later mounted in the cold space. Temperatures of the first four stages run at about $167 \mathrm{~K}, 70 \mathrm{~K}, 27 \mathrm{~K}$, and $15.5 \mathrm{~K}$. The compressor cylinder and piston, with $90 \mathrm{~cm}^{3}$ displacement, were larger than, but otherwise similar, to those shown previously (12). The crank and connecting rod mechanism from a small air compressor were adapted to drive the piston and displacer of the cryocooler at $1.1 \mathrm{~Hz}$. The relative phase between piston and displacer motions was adjusted empirically for minimum temperature.

Displacer dimensions in Fig. 3 are given in $\mathrm{mm}$, and other dimensions, such as wall thicknesses of the glass-epoxy tubing, are drawn in correct proportions. The displacer is shown in its lowest pusition so the compression space is at maximum volume and the five expansion spaces are at zero volume. The assembly is shown as it should appear at low temperature. To achieve lowest temperature, it is essential to allow for differential contraction of the nylon and the glass-epoxy, to ensure that the lower expansion spaces close up completely at the bottom of the displacer stroke. It is less important for the upper expansion spaces to close completely. We determined that the differential contraction between room temperature and cyogenic temperatures was almost exactly $1 \%$. We accordingly made the four lower displacer sections about 1.1\% longer, at room temperature, than the corresponding cylinder sections, so that the lowest expansion space would go to zero volume at the bottom of the displacer stroke while the upper expansion spaces would have successively larger, but still small, finite mimimum volumes. 


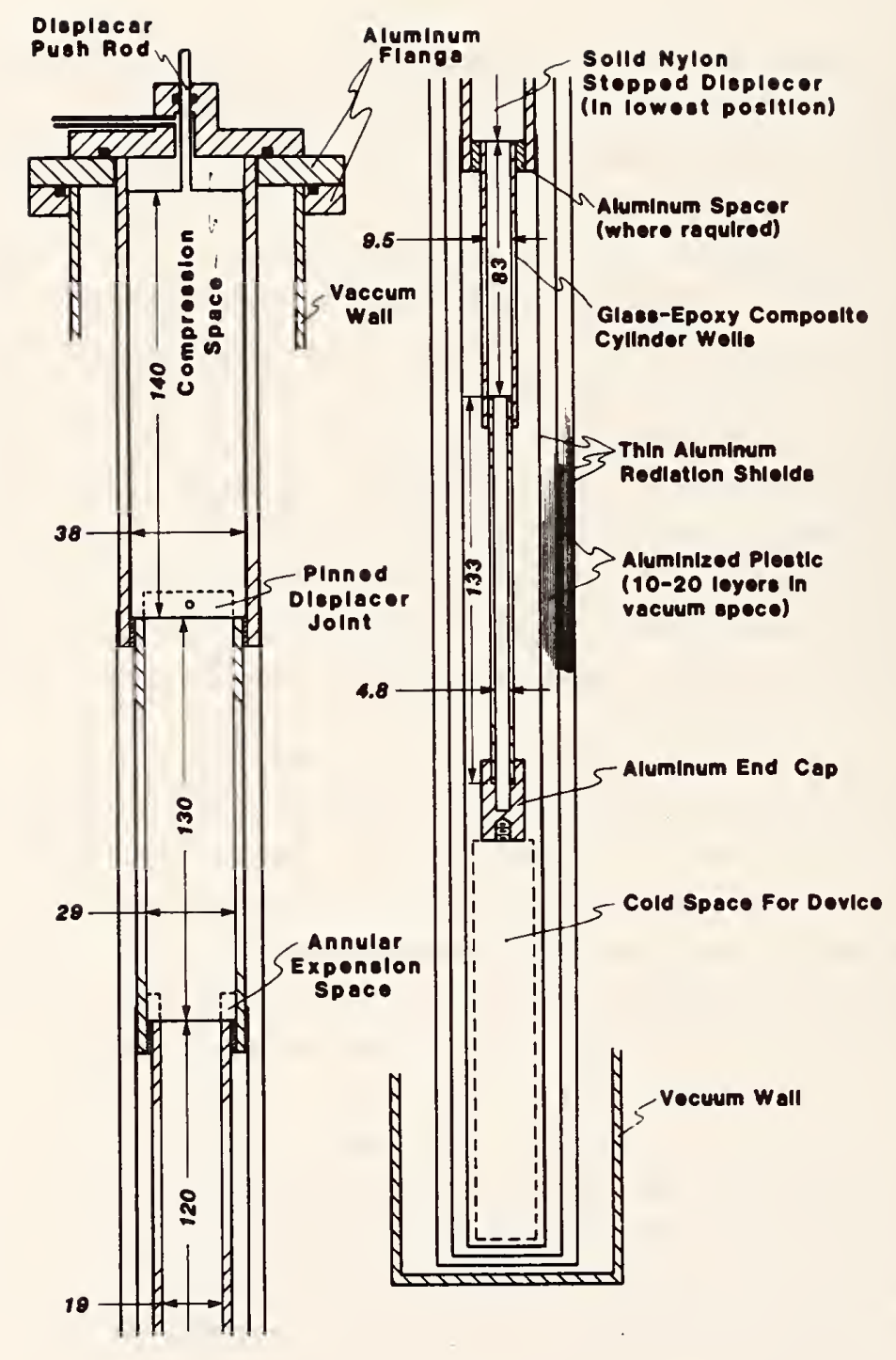

Fig. 3. Scale drawing of NBS 5-stage cryocooler. 


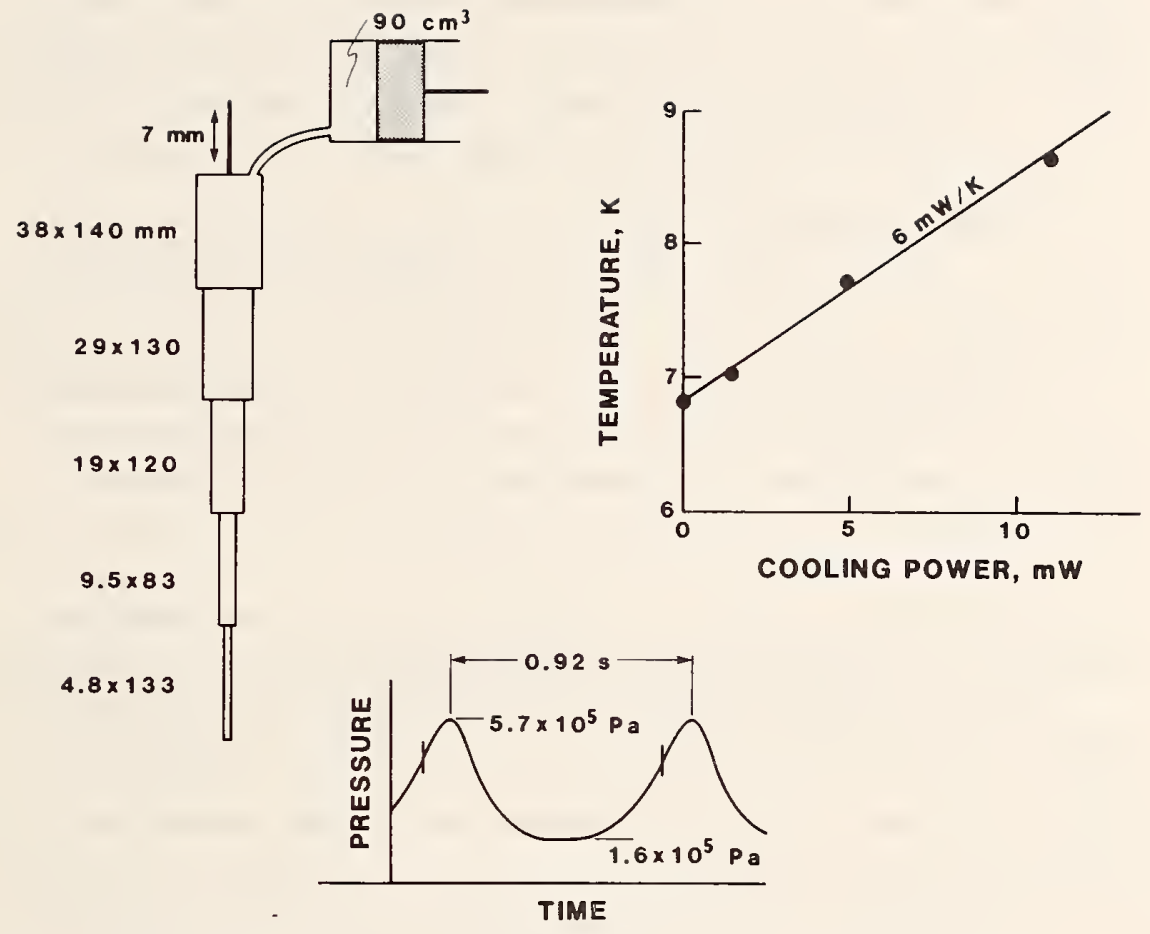

Fig. 4. Displacer and cylinder dimensions (upper left), temperature vs. heat input to cold end (right), and pressure variation during two cycles of operation, with short vertical marks indicating time of displacer bottom-dead-center position (bottom).

With regard to radial dimensions, the four lower displacer sections were simply cut for an easy push fit (at room temperature, probably 10 to $20 \mu \mathrm{m}$ radial clearance.) Differential contraction provides plenty of radial clearance, (probably more than optimum) when the machine is cold. On the first (upper-most) section, a uniform taper from about 20 to $100 \mu \mathrm{m}$ radial clearance, bottom to top, was provided.

It is convenient, for several reasons, to make the displacer in five individual pieces and pin them together as indicated in the figure. In particular, it is much easier to fit the 
individual sections to the corresponding cylinder, and a mistake in one section does not waste the whole displacer. Also, joints between sections can be made slightly loose to permit self-alignment of each section in the cylinder.

If a displacer section fits too tightly, that section may heat up due to friction when the machine is started at room temperature. The remedy, obviously, is to remove a few $\mu \mathrm{m}$ of material from the offending section. Copper-constantan thermocouples were used to measure temperatures of the upper, warmer stages and 1/8-W carbon resistors for the lower stages.

Some performance data are given in Fig. 4. The vertical coordinate in the graph of cooling power is the temperature of an aluminum cylinder in the cold space, serving as a dummy load. During the last week before this conference, a SQUID gradiometer has been operating in the cryocooler. Performance of the system will have to be left for a later publication. This work is being done in collaboration with D. B. Sullivan.

\section{References}

1. Letter from the Editor, "Opportunities for Miniature Cryocooler Research and Development". Cryogenics 30 , 164 (1980).

2. Zimmerman J. E., and Flynn, T., eds.: Applications of Closed-Cycle Cryocoolers to Small superconducting Devices. NBS Special Publication SP-508, superintendent of Documents, U. S. Government Printing office, Washington, D. C. 20434 (1978). (Also available from the authors).

3. Proc. Cryogenic Cooler Conf., USAF Academy, Co., oct. 1973, Tech. Report AFFDI-TR-73-149, Vol. 1, wright- Patterson Air Force Base, Ohio 45433 (Dec. 1973).

4. Radebaugh, R.: See Ref. $2, \mathrm{p} .7$.

5. Sherman, A.: Astronautics and Aeronautics 39, Nov. (1978). 
6. Zimmerman, J. E., and Sullivan, D. B.: Cryogenics 19 , 170, (1979).

7. Steyert, W.: See Ref. 4, p. 7.

8. Barclay, J. A.: Cryogenics (to be published).

9. Pratt, Jr., W. P., et al.: Cryogenics 17, 689 (1977). See also Reports LA-8111 and LA-8134, Los Alamos Scientific Laboratory, Los Alamos, NM 87545.

10. Radebaugh, R.: See Ref. 2, p. 93.

11. Rule, T. T., and Qvale, E. B.: Adv. in Cryogenic Engineering, K. Timmerhaus, ed., V14, 343 (1969), Plenum Press.

12. Zimmerman, J. E., and Radebaugh, R.: See Ref. 2, p. 59.

13. Feller, D. W.: Electronic Design 23, 116 (1974).

14. Zimmerman, J. E. and Campbell, W.: Geophysics, 40, 269 (1975).

15. Campbell, W, and Zimmerman, J. E.: IEEE Trans. Geosci. Elect. (1980) (Accepted for publication).

16. Zimmerman, J. E., et al.: IC SQUID, edited by Hahlbolm and Lübbig.

17. Sullivan, D. B., and Zimmerman, J. E.: Int. J. of Refrig. 2, 211 (1979).

18. du Pré, F. K., and Daniels, A.: Prog. in Refrig. Sci. and Tech., Vol. 1, AVI Publishing Co., 137 (1973).

19. Little, W.: See Ref. 2, p. 75.

20. Little, W.: AIP Conf. Proc. No. 44 , edited by Deaver et al., (1978).

21. Gasser, et al., Applications of Cryogenic Technology, Vol, 4, 416 (197i).

22. Benson, G.: U. S. Patent Nos. 3, 928, 974 and 4,044,558.

23. Benson, G.: Proc. 12th IECED, 416 and 1478 (1977).

24. Brenckenridge, R. W. et al.: Technical Report AFFDL-TR-78 -166, Wright-Patterson Air Force Base, OH 45433 (Dec. 1978).

25. Sherman, A., et al.: in Advances in Cryogenic Engineering, ed. by Timmerhaus, Clark, and Reed, V25. Plenum Press (to be published). 
"Refrigeration for Cryogenic Sensors and Electronic Systems," NBS Special Publication SP-607, (J. E. Zimmerman, D. B. Sullivan, and S. E. McCarthy, eds.), Superintendent of Documents, U. S. Government Printing Office, Washington, D. C. 20234 (1981), pps. 186-194.

OPERATION OF A PRACTICAL SQUID GRADIOMETER IN A LOW-POWER STIRLING CRYOCOOLER ${ }^{\dagger}$

\author{
D. B. Sullivan, J. E. Zimmerman, and J. T. Ives $^{\dagger+}$ \\ National Bureau of Standards \\ Electromagnetic Technology Division \\ Boulder, C0 80303
}

\begin{abstract}
A commercial Nb point-contact SQUID has been used in the construction of a simple magnetic gradiometer system supported by a split Stirling refrigerator. The magnetic interference generated by the cryocooler was sufficiently small to permit the acquisition (for demonstration purposes) of a reasonable magnetocardiogram. Preliminary investigation of the source of the residual refrigerator interference indicates that cyclic temperature variation is the largest single factor influencing the gradiometer output. A simple electronic temperature controller has been added to a different cryocooler to study temperature stabilization. Better than $1 \mathrm{mK}$ stability has been achieved at $8.5 \mathrm{~K}$.
\end{abstract}

Key words: Cryocoolers; low temperature; magnetic gradiometer, refrigeration; SQUID; Stirling cycle; superconductivity.

\title{
1. Introduction
}

The economic viability of superconducting instrumentation would be greatly enhanced if properly matched cryocoolers were readily available to support their operation. By properly matched we mean: 1. cost comparable to or less than the supported instrument, 2 . cooling capacity not significantly greater than that required (often only a few milliwatts), 3. package size comparable to or not much larger than that of the device in a liquid-helium cryostat, and 4 . vibration and magnetic interference levels which do not appreciably affect the instrument operation. This last requirement is especially critical for SQUID magnetometers and magnetic gradiometers which are designed to sense environmental magnetic fields or gradients (e.g., geophysical ffields or biological fields). The SQUID magnetometer has a sensitivity of the order of $10^{-10}$ times the earth's magnetic field and thus extremely small angular vibrations translate directly into interference if the device is operated in such a field. In this paper we discuss this and other potential interference sources and describe a SQUID gradiometer supported by a low-power cryocooler [1,2]. As a demonstration of the level of interference for this system, a magnetocardiogram was recorded.

\section{Cryocooler}

The cryocooler used in this work has been recently described by Zimmerman [2]. Figure 1 , taken from this reference, shows the details of construction of the cold end of this split Stirling refrigerator. The refrigerator operating at $1 \mathrm{~Hz}$ has achieved a temperature of $7 \mathrm{~K}$ with no heat load (an aluminum rod occupied the 'cold space for device' shown in Figure 1). The compressor displacement is $90 \mathrm{~cm}^{3}$ and optimum operating helium pressure is just over 2 atmospheres (gauge pressure). The displacer stroke is $0.7 \mathrm{~cm}$.

Tontribution of the U. S. Government, not subject to copyright. Work supported by the Navy Coastal Systems Laboratory.

t+ Present address: Engineering Department, University of Utah, Salt Lake City, Utah. 


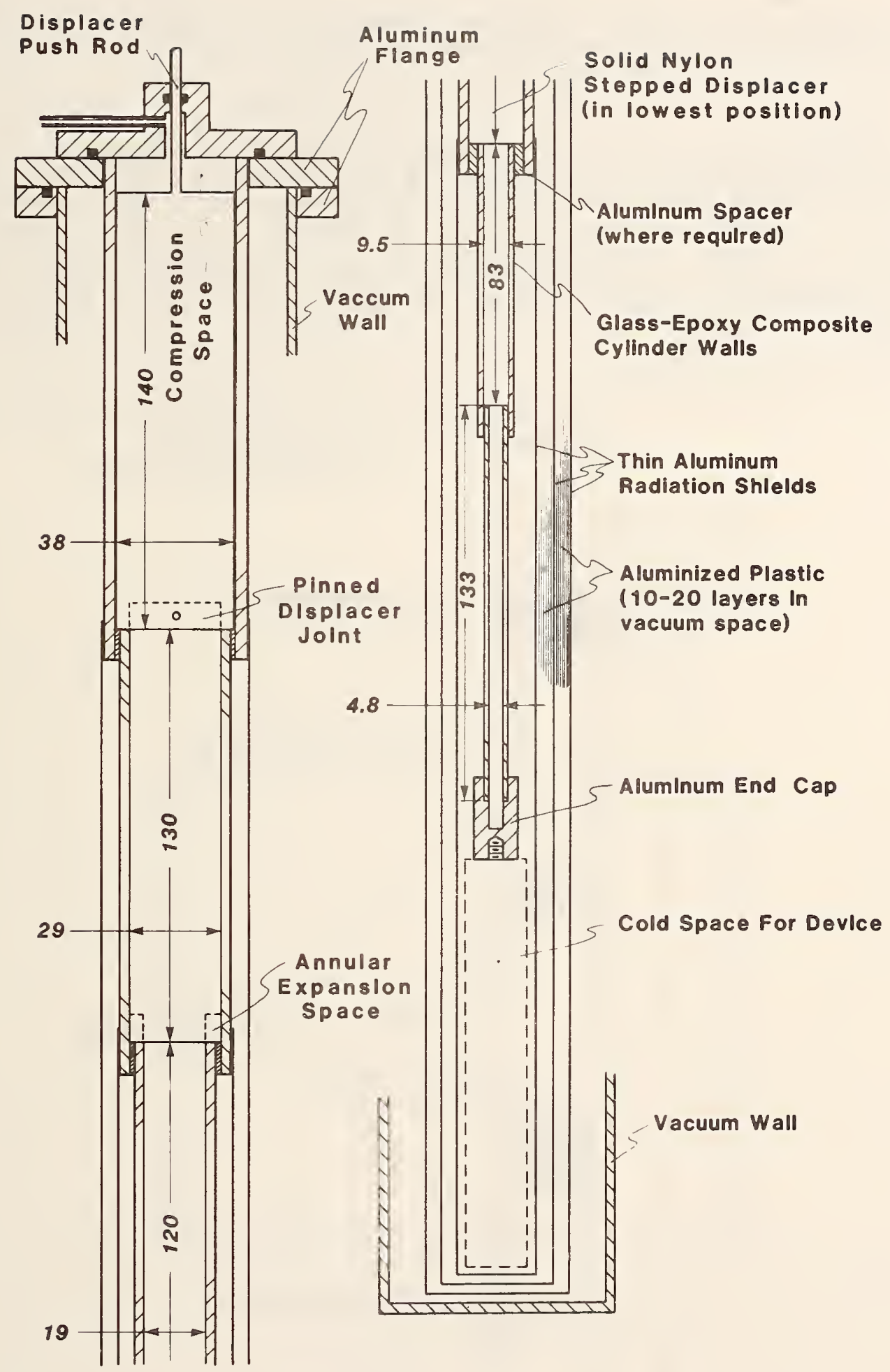

Fig. 1. Details of 5-stage; split Stirling cryocooler. Dimensions are in millimeters. Figure taken from reference [2]. 
The selection of many of the design features for the cryocooler was influenced by interference considerations. These design features are described in section 4 below under the appropriate potential interference sources.

\section{SQUID Gradiometer}

The gradiometer and its principal dimensions are shown schematically in figure 2. The diameter of the gradiometer was selected such that none of the shields of its support refrigerator (Figure 1) would need modification. This was done to keep the system as simple as possible, but the resulting gradiometer was not particularly sensitive with the limited diameter and baseline. The commercial toroidal SQUID is centered within a niobium tube between the end coils. This tube is moved vertically to achieve axial balance. Small superconducting tabs were attached to the tube (in pairs, one at each end) to achieve transverse balance.

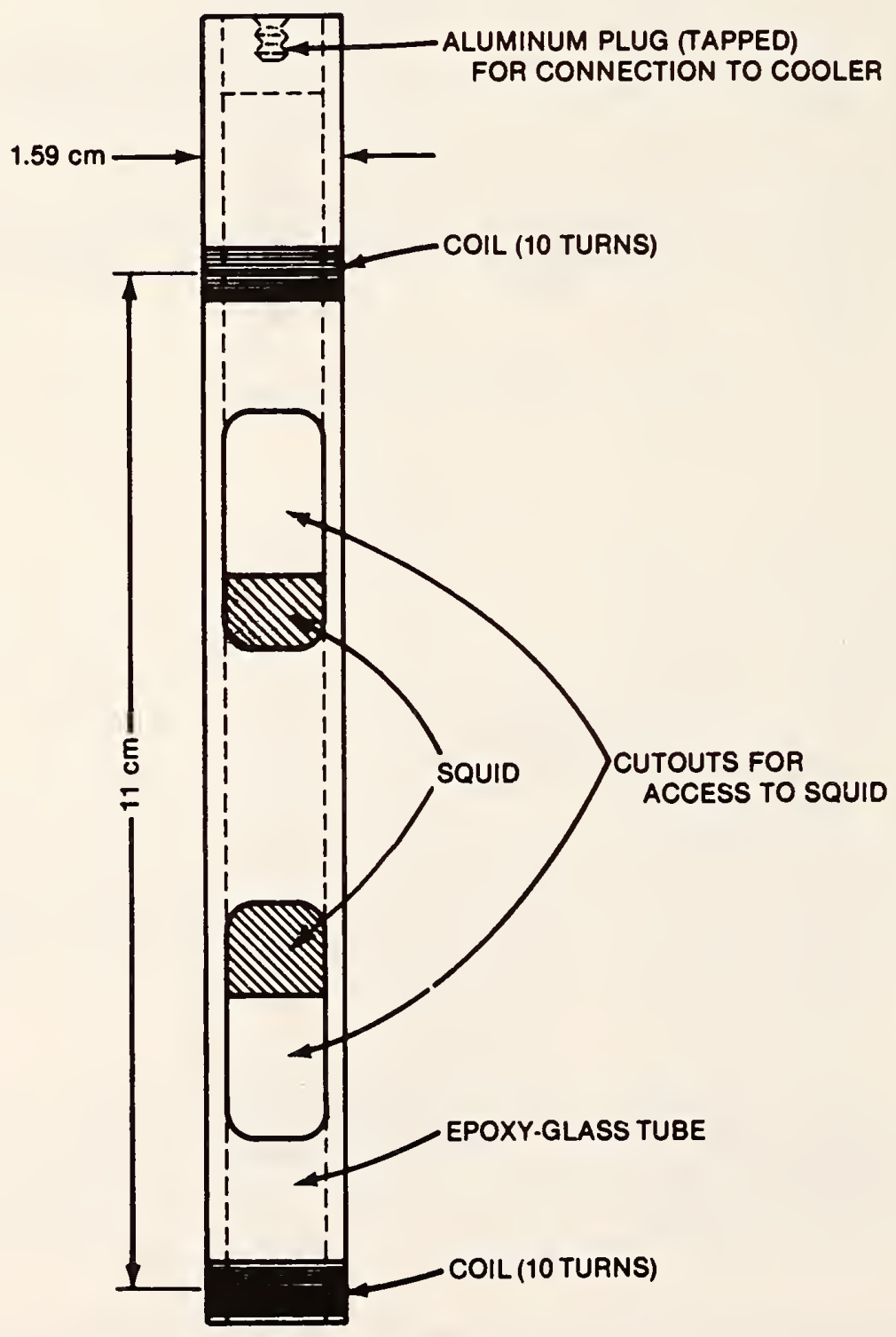

Fig. 2. SQUID gradiometer. The SQUID is inside a niobium cylinder. 
The system was balanced to approximately $1 \times 10^{-4}$ both axially and in the transverse direction while operated in liquid heliup The sensitivity was found to be $210^{-9} \mathrm{~T} / \mathrm{m}$ per flux quantum with a noise level of $\sim 10^{-11} \mathrm{~T} / \mathrm{m}$ in a $40 \mathrm{~Hz}$ bandwidth. For thermal anchoring, four strips of aluminum foil $(4 \mathrm{~mm}$ wide by $0.3 \mathrm{~mm}$ thick) were tied along the entire length of the gradiometer with the ends also tied to the aluminum cold end of the refrigerator. The SQUID was also independently anchored (thermally) to the cold end. The gradiometer coils (10 turns each) were connected in parallel to the SQUID rather than the usual series connection. This was done so that more turns could be used on each coil and still have the combined inductance match that of the SQUID coil.

For the interference tests the gradiometer was operated partly within an aluminum shieided room [3], as shown in figure 3. The shielded room provides some isolation between compressor and displacer since its cutoff frequency is $1 / 3 \mathrm{~Hz}$. The cylinder with displacer was mounted rigidly to the roof of the room to avoid any rocking motion induced by the rotation of the displacer drive shaft (see Fig. 3). This rigid mounting led to additional microphonic noise in the 3 to $10 \mathrm{~Hz}$ range. Vibrations in this frequency range were generated by some heavy refrigeration equipment in the building.

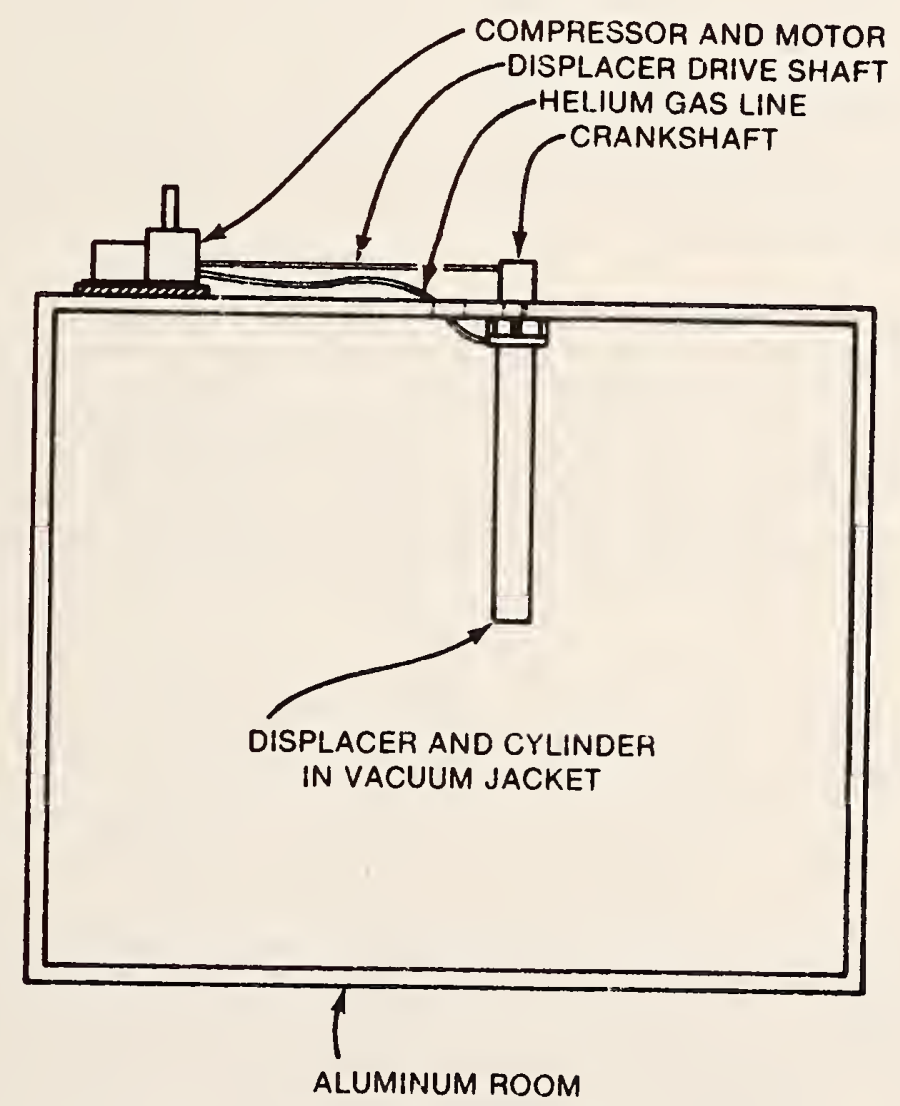

Fig. 3. Mounting of the cryocooler in an aluminum shielded room. 


\section{Potential Sources of Interference}

In previous papers we emphasized the refrigeration aspects of the cryocooler while alluding to certain characteristics which would minimize the magnetic signature of the device. In this section we expand upon the design features relating to magnetic interference.

\subsection{Motional Interference}

The use of low speed (in the neighborhood of one $\mathrm{Hz}$ ) and the low power requirement result in low vibrational noise, because of the low static and dynamic forces involved. With the plastic regenerator materials, this low-speed is, in fact, essential since much higher speed operation reduces the thermal penetration depth and thus results in regenerator inefficiency, particularly in the bottom stage.

The displacer requires yery little power for operation, so the mechanical components which produce the displacer motion can be very smooth in operation. The compressor and motor are coupled to the cold portion of the cooler through a drive shaft and a tube through which the helium gas is transported between compressor and displacer sections. It is essential that viuration transmission through these two connections be minimized. To this end we use rather light weight and flexible materials (thin-wall brass for the drive shaft and small diameter copper tube for the helium gas) for these components. By separating the compressor and displacer one meter from one another we find little vibration transmission from compressor to displacer. Additional flexibility and versatility might be achieved through use of a simple hydraulic system to transmit the mechanical motion from the motorcompressor to the displacer.

The one component of motion which still remains as a potential problem is that of pressure flexing of the displacer cylinder. It is unlikely that this cylinder is perfectly uniform around its circumference and the cyclic pressure variations could therefore result in some cyclic transverse motion of the cold end. This problem would be reduced by operation at the lowest possible pressure.

An earlier paper [1] described the results of operation of a simple SQUID magnetometer in a four-stage cooler. The peak-to-peak signature at the cooler frequency of $]_{5} \mathrm{~Hz}$ was $0.5 \mathrm{nT}$ (SQUID operated in the earth's field). This corresponds to a motion of $10^{-5}$ radian if this is the only interference source, emphasizing the extreme stability required by this type of instrument. This field oscillation was dependent upon the orientation of the SQUID in the horizontal plane, so we concluded that at least part of that signature was due to flexing of the displacer cylinder presumably induced by the pressure oscillations. There was no direct evidence for other interference mechanisms, but they could easily have been present as well.

For magnetic gradiometers, motional interference is often not as severe. If the earth's field is uniform in the vicinity of the device (the usual case), the motional signature is reduced by the degree of balance of the gradiometer. That is, it is the remnant magnetometer response which picks up the field motion. In this particular case the gradiometer is balanced to a part in $10^{4}$ so motional inteference is reduced by the same factor.

\subsection{Compressor Interference}

It is necessary to minimize direct magnetic interference by the compressor at the cold end. Two precautions are taken. First, the compressor is designed with a minimum of ferromagnetic components. Materials utilized included brass, aluminum, stainiess steel, and nylon. We have used bronze and nylon bushings rather than ball and roller bearings which are commonly ferromagnetic. The piston is nylon in a stainless tube. The motor and gearbox selected for the machine has cast aluminum housing components and appears to have only a modest magnetic signature (this motor was once operated within $15 \mathrm{~cm}$ of the gradiometer with no severe interference). The second precaution involves modest separation (1/2 to 1 meter) of the motor-compressor and the displacer sections of the refrigerator. It is conceivable that some sort of shielding could be added to the motor and compressor to further reduce their magnetic signature, but this does not yet appear to be a major interference source. 


\subsection{Magnetism of Cold End Materials}

Plastics were chosen for the critical cold parts of the refrigerator because they are nominally nonmagnetic. However, at the sensitivity level of the SQUID, the magnetic properties of virtually all materials are not negligible. Small remanent moments and magnetic susceptibilities often result in readily measured effects. The materials concerns fall into two categories, magnetic interference from moving components and slow drifts which arise from temperature dependent magnetic effects in materials which are fixed relative to the SQUID.

In the first instance two phenomena are important. The presence of a remanent magnetic moment in the displacer leads to an obvious magnetic signature. In general, remanent magnetism in such materials is associated with traces of ferromagnetic substances either introduced into the bulk of the material during manufacture, or quite commonly, by machine work during shaping of the component. The latter contamination can be reduced sharply by careful cleaning procedures. Remaining permanent moments can be reduced by demagnetizing procedures. Even without remanent magnetism, moving components can generate a magnetic signature since the ambient field (often the earth's field) induces a moment in anything with a non-zero magnetic susceptibility.

It is difficult to be quantitative in the case of remanence, but we can estimate the size of the induced moment at the bottom end of the nylon displacer. Assuming for the moment that the lowest part of the displacer produces the strongest effect, we suggest that the displaced volume in the bottom stage is the relevant volume of nylon to consider. That is, we assume that the effect of cyclic axial motion of a semi-infinite rod in a magnetic field is the same as the cyclic appearance of a volume of the rod equal to the cross sectional area times the stroke. Thus for the 5-stage cooler of Eigure 3 , we have a volume of $0.7 \mathrm{x}$ $\pi \times(2.4)^{2} \mathrm{~cm}^{3}$. For nylon with a susceptibility of $10^{-5}$ in a field of $5 \times 10^{-4} \mathrm{~T}$ this yields a cyclic induced moment of $\tilde{5} \times 10^{-14} \mathrm{Am}^{2}$. This translates to an interference gradient field of approximately $10^{-14} \mathrm{~T} / \mathrm{m}$. (The spacing between the end of the displacer and the top coil of the gradiometer is $\sim 3 \mathrm{~cm}$ ). This is well below any interference level which might be achieved in the near future.

The magnetic susceptibility of many materials can be a rather strong function of temperature leading to long term drift in the induced magnetic moment. The refrigerator components most likely to experience such temperature drift are the radiation shields and mylar superinsulation. Drifts of many degrees $C$ in temperature are likely since radiation heat transfer is very small and the time constant for temperature equilibrium might then be extremely long. Lacking specific knowledge of either the magnitude of such temperature variation or the temperature dependence of the relevant material susceptibilities, it is not practical to dwell on this question. Needless to say, the appearance of drift might eventually lead us to attemp to clarify this situation.

\subsection{Temperature Variation}

Besides this last effect (induced magnetism which drifts with temperature), there is a more direct influence which temperature change can have on a SQUID. If the critical current of the SQUID is a function of temperature, then temperature change will have an effect on the operating point of the SQUID. In principle this should not change the output of a SQUID operated in a phase-locked loop, but measurements indicate that this is not the case. This relation between SQUID output and operating point ( $r f$ level or critical current) is probably a second order effect related to some asymmetry in the interference pattern. It's presence means that temperature changes (either drift or oscillatory changes) can affect the SQUID output directly. The cures for this problem are twofold. First eliminate the temperature variations and second, eliminate (or reduce) the temperature dependence of the SQUID's critical current. Probably both solutions should be considered.

\section{Interference Measurement}

The gradiometer sensed an interference signal at $1 \mathrm{~Hz}$ (compressor frequency) with an amplitude of $\sim 6 \times 10^{-11} \mathrm{~T} / \mathrm{m}$. We conducted several experiments to isolate the source of the noise. First, we placed a high permeability shield around the SQUID and found no 
observable change in the amplitude of the signature. This immediately eliminates compressorgenerated magnetic signals as the predominant interference source. Furthermore, this shield attenuated the earth's field by at least a factor of 100 . The lack of any change in interference with this shielding suggests that both gradiometer motional noise and induced moments (non-zero susceptibility) are also unimportant at this level. Finally, we noted rapid drift when the cooler was turned off momentarily. During such shutdown the SQUID remained fully in operation. This suggests a strong dependence of SQUID output on temperature as discussed above. From direct measurements we find that the temperature of the bottom end varies by at least several $\mathrm{mK}$ at the refrigerator frequency and previous measurements on the SQUID indicated a strong dependence of critical current on temperature. Thus we conclude that the primary source of the apparent magnetic signature at this level is temperature fluctuations at the Souid. This is also consistent with the observation that the signal amplitude is somewhat dependent on temperature since the slope of the $I_{C}$ vs. T curve is a function of $T$ and some dependence is thus expected.

We also noted that the very low frequency noise (less than $1 \mathrm{~Hz}$ ) was small. After achieving temperature equilibrium we noted a rather stable signal (less than $2 \times 10^{-11} \mathrm{~T} / \mathrm{m}$ variation over a period of three minutes). This could probably be improved through temperature regulation which will be discussed in the next section.

\section{Interference and Drift Reduction}

We usea a very simple expedient to cancel most (90\%) of the $1 \mathrm{~Hz}$ signature. This was to attach a small magnet to the shaft of the compressor so as to generate a field which just canceled the observed signature. The proper way to deal with this sort of interference reduction is to use a commutative filter with a reference derived from the compressor shaft. The advantage of such a filter is that it can also handle harmonics of the fundamental interference which are clearly present. Nonetheless, the simple cancellation of most of the interference allowed the following measurement.

As a demonstration of the system we recorded two magnetocardiograms (see Figure 4 ). The first was taken with the gradiometer in a simple cryostat and the second with the gradiometer supported by the 5-stage cooler. Bandwidth for the measurements was 2 to 40 $\mathrm{Hz}$ (to further reduce the one $\mathrm{Hz}$ signature). The added noise in the 3 to $10 \mathrm{~Hz}$ range is probably a result of the rigid mounting to the shielded room (see Section 3 ).

a)

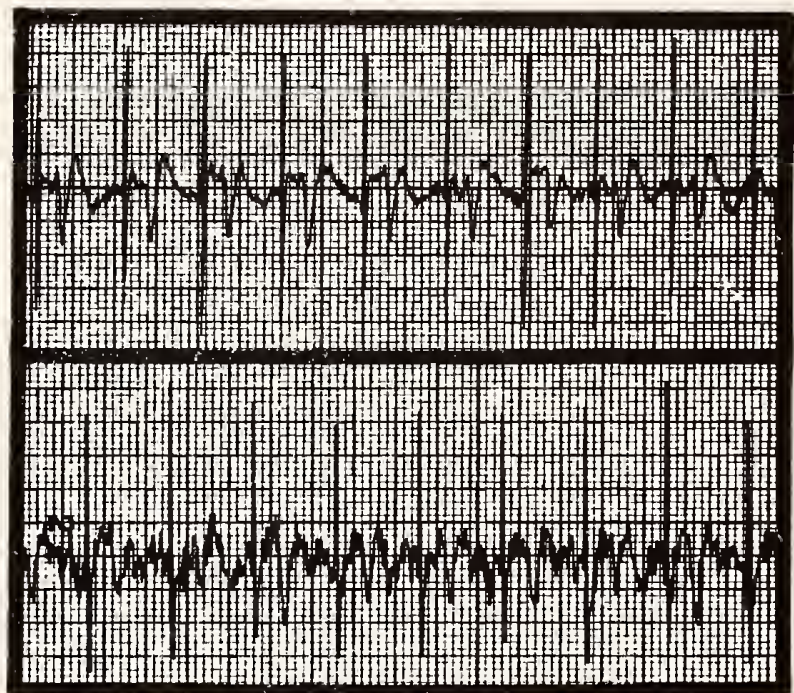

\section{$\stackrel{\text { IN }}{\text { CRYOSTAT }}$}

IN

CRYOCOOLER

Fig. 4. Magnetocardiograms taken with gradioneter in a simple cryostat (a) and in the 5-stage cryocooler (b). 
This result provides a fairly clear indication that mechanical refrigerators can be compatible with SQUID instruments, although tiere is room for much improvment. Obviously, the problem of temperature fluctuation and drift must be addressed first. (The reduction of SQUID temperature sensitivity will also help, but we assume that complete elimination of such temperature sensitivity is very difficult).

We have experimented with two means for stabilizing the temperature of the cryocooler. These experiments have been carried out with a 4-stage Stirling cryocooler, the bottom end of which is shown in Figure 5. The carbon-resistance thermometer and the heater were used as sensor and control elements in a simple electronic temperature regulator (servo system). This system was quite effective in eliminating drift. Over a period of two hours, the temperature change was no more than a fraction of a mK with the temperature at $8.5 \mathrm{~K}$. Because of the rather slow thermal response, the simple regulation circuit did not significantly reduce the $2 \mathrm{mK}$ oscillation (at $1 \mathrm{~Hz}$ ) which was observed without the regulator. Further improvement of this regulation is clearly needed. One should note that alternating current at a frequency above the SQUID sense band can be used for both the heater and thermometer to eliminate any possible interaction between the SQUID and a direct current in these elements.

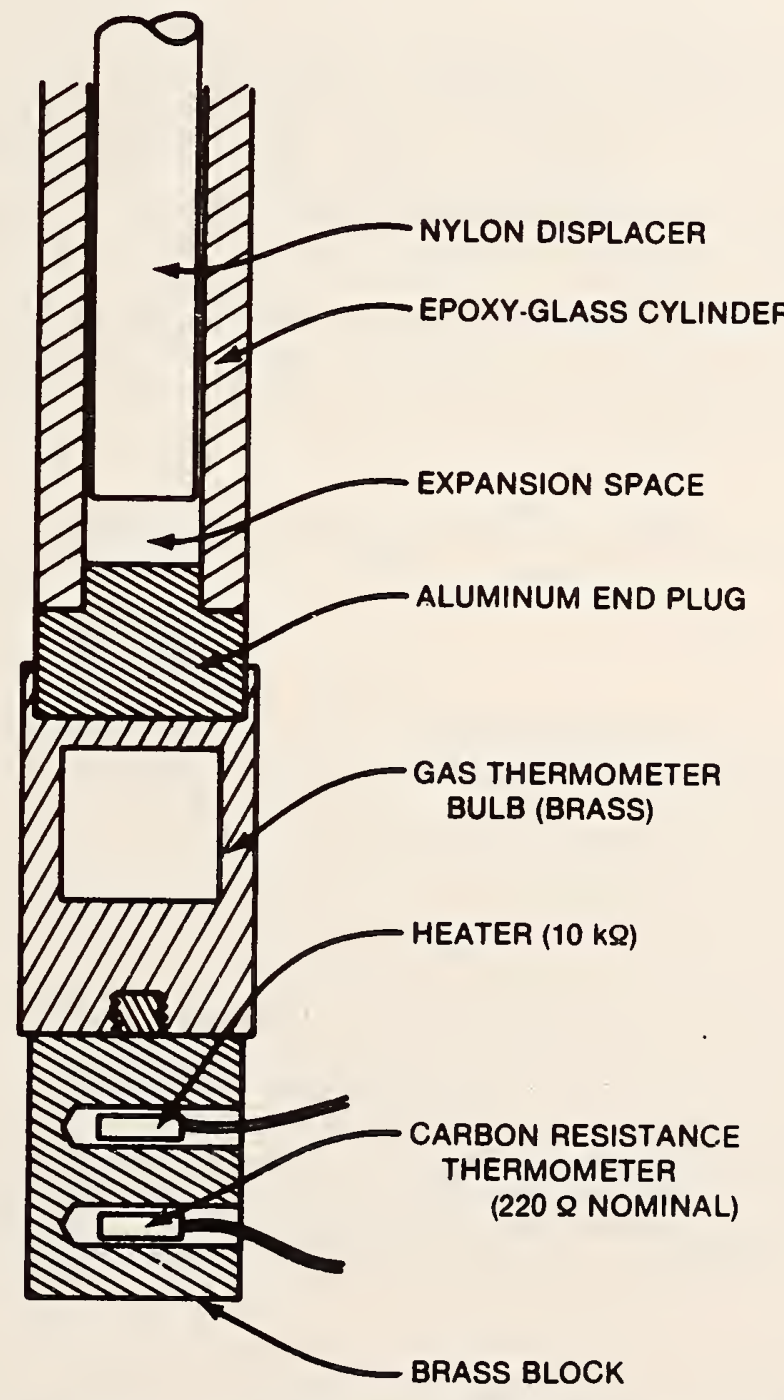

Fig. 5. Details of the bottom end of the 4-stage cryocooler with thermometer and heater for electronic temperature control. The helium gas bulb permits control of the thermal mass of this bottom stage. 
The helium gas bulb shown in Figure 5 provides a simple means for changing the heat capacity of the cold end of the refrigerator. Even though the volume of this bulb is only $1 \mathrm{~cm}^{3}$, the total heat capacity of the bottom stage could be increased by a factor of 10 by pressurizing it with helium gas to only 4 or 5 standard atmospheres. This results in a tenfold reduction in the oscillatory temperature variation and significantly reduces drift even without the electronic temperature regulator.

\section{Summary}

In summary then, a SQUID gradiometer supported by a split Stirling cryocooler has been shown to operate at an interference level sufficient to obtain a magnetocardiogram of modest clarity. Temperature fluctuations produce the primary interference signature and methods for temperature stabilization have been studied. We expect that with each improvement which reduces magnetic interference, we will identify other interference sources which affect the system at a lower level. The most logical approach is to proceed to deal with these as they are uncovered.

\section{References}

[1] J. E. Zimmerman and R. Radebaugh in "Applications of Closed-Cycle Cryocoolers to Small Superconducting Devices," NBS Special Publication SP-508, J. E. Zimmerman and T. M. Flynn, eds.), Superintendent of Documents, U. S. Government Printing Office, Washington, D. C. 20234 (1978). (Also available from the authors), p. 59.

[2] J. E. Zimmerman in "IC-SQUID (Conference)" Berlin, West Germany, May 1980 (to be published).

[3] J. E. Zimmerman, J. Appl. Phys. 48, 702 (1977). 
"Refrigeration for Cryogenic Sensors and Electronic Systems," NBS Special Publication SP-607, (J. E. Zimmerman, D. B. Sullivan, and S. E. McCarthy, eds.), Superintendent of Documents, U. S. Government Printing Office, Washington, D. C. 20234 (1981), pps. 173-177.

\title{
MEASUREMENT OF THERMAL PROPERTIES OF CRYOCOOLER MATERIALS ${ }^{\dagger}$
}

\author{
J. E. Zimmerman, D. B. Sullivan, R. L. Kautz, and R. D. Hobbs ${ }^{\dagger+}$ \\ National Bureau of Standards \\ Electromagnetic Technology Division \\ Boulder, CO 80303
}

\begin{abstract}
Materials used in several low-power cryocoolers are characterized by very low thermal conductivities and moderate to large heat capacities at low temperature. Consequently, thermal equilibrium times are inordinately long for measurements of thermal properties to be made by the usual quasi-steady-state methods. We have developed a method of measurement in which the thermal conductivity and the specific heat are derived from observations of the time-dependent temperatures at two or more points on a cylindrical sample in response to a step function of heat applied to one end of the sample. Some preliminary results have been obtained on G-10 epoxy laminated tubing. These transient measurements give results in much less time than the steady-state method, and have the.additional advantage of being adaptable, with fair accuracy, to the cryocooler structure itself, or to a separate structure geometrically similar to the cryocooler.
\end{abstract}

Key words: Composites; cryocoolers; plastics; refrigeration; regenerators; specific heat; thermal conductivity.

\section{Introduction}

Measurement of thermal properties of plastic and composite materials used in the construction of several low-power-cryocoolers requires long times if done by conventional methods. For example, a sample of nylon whose longest dimension is $5 \mathrm{~cm}$ would require something like two hours to reach thermal equilibrium after an input of heat at one end. This estimate is based on a thermal conductivity $K \sim 0.5 \mathrm{~mW} / \mathrm{cm} \mathrm{K}$ and a specific heat $c_{0} \sim$ $25 \mathrm{mu} / \mathrm{cm}^{3} \mathrm{~K}$ at $10 \mathrm{~K}$. Thermal equilibrium times will of course be proportionately grealer for special composite materials with large heat capacities. We have therefore developed a method of measurement in which we apply a step function in heat flow to one end of a cylindrical sample and observe the propagation of the transient in temperature as the heat diffuses along the sample. Thus, thermal properties of the sample material are derived from measurements made during the initial development of the transient, rather than after the transient has died out as in conventional equilibrium or steady-state ac methods [1]. This gives an order of magnitude reduction in time required for the measurements, not to mention the fact that both the thermal conductivity and the specific heat are derived from the same set of measurements. In addition, the method can be used for rough measurements on the cryocooler structure itself, the geometry being appropriate.

Fontribution of the U. S. Government, not subject to copyright. Work supported by the Office of Naval Research.

t+Present address: Physics Department, Indiana State University, Terre Haute, Indiana. 
Transient measurements of thermal properties have been made previously on cylindrical samples by a heat pulse method. Our reasons for using a step function rather than a pulse was that an ideal heat pulse momentarily raises the end of the sample to a high temperature, thereby enhancing the possibility of heat loss by extraneous mechanisms such as radiation, and also complicating the analysis through the temperature dependence of the thermal conductivity and specific heat. The magnitude of the step function can be adjusted to give a slow, steady, and relatively small, increase in temperature of the end of the sample.

\section{Analysis of the Method}

The ideal geometry is a uniform semi-infinite bar with a heater on the free end, $x=0$, and thermometers to measure temperatures $T_{1}$ and $T_{2}$ at two points $x_{1}$ and $x_{2}$ (Fig. 1). The simplest initial condition is to let the bar come ${ }^{2}$ to thermal equilforium at a temperature $T$, and then at time $t=0$, turn on the heater at a power level $P$. The one-dimensional diffusion equation for this situation is

$$
\frac{\partial^{2} T}{\partial x^{2}}=\frac{C}{k} \frac{\partial T}{\partial t}
$$

where $k$ and $C$ are respectively the thermal conductivity and specific heat per unit volume of the material. The solution for the stated initial condition is:

$$
T(t, x)=T_{0}+\frac{2 P}{A \sqrt{\pi k C}} \sqrt{t} e^{-C x^{2} /(4 k t)}-\frac{P}{k A} \times \operatorname{erfc}\left[\left(\frac{C x^{2}}{4 k t}\right)^{1 / 2}\right],
$$

where erfc means the complementary error function, and $A$ is the cross-sectional area. This solution is plotted as a function of $x$ and of $t$ in Fig. 2 .

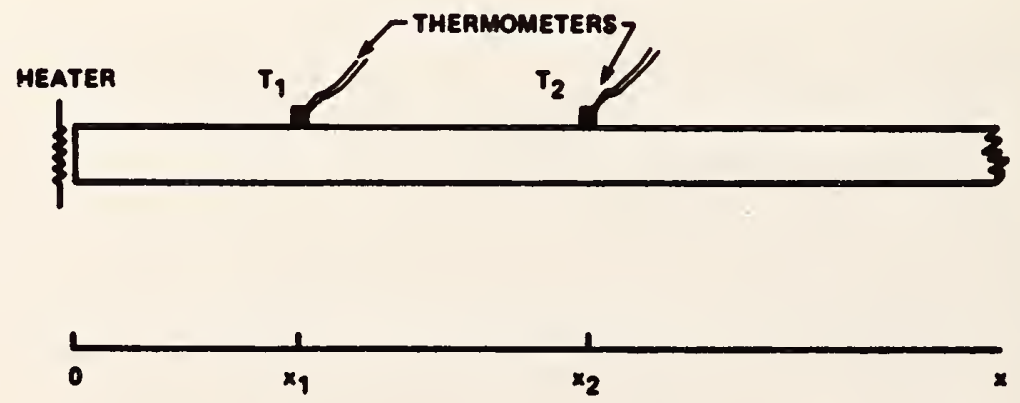

Fig. 1. Experimental arrangement for thermal properties measurement. The heater is turned on abruptly at $t=0$ (step function) and the resultant temperature variation (sensed by the two thermometers) provides the means for determining the thermal conductivity and specific heat. The right hand end is connected to a heat sink, the temperature of which is controlled to set the equilibrium value of the temperature at $T_{0}$. The sample is suspended in a vacuum and surrounded by a liquid helium cooled shroud to minimize heat leaks to it. The leads from the carbon resistance thermometers are heat sunk to this shroud for similar reas ons. 


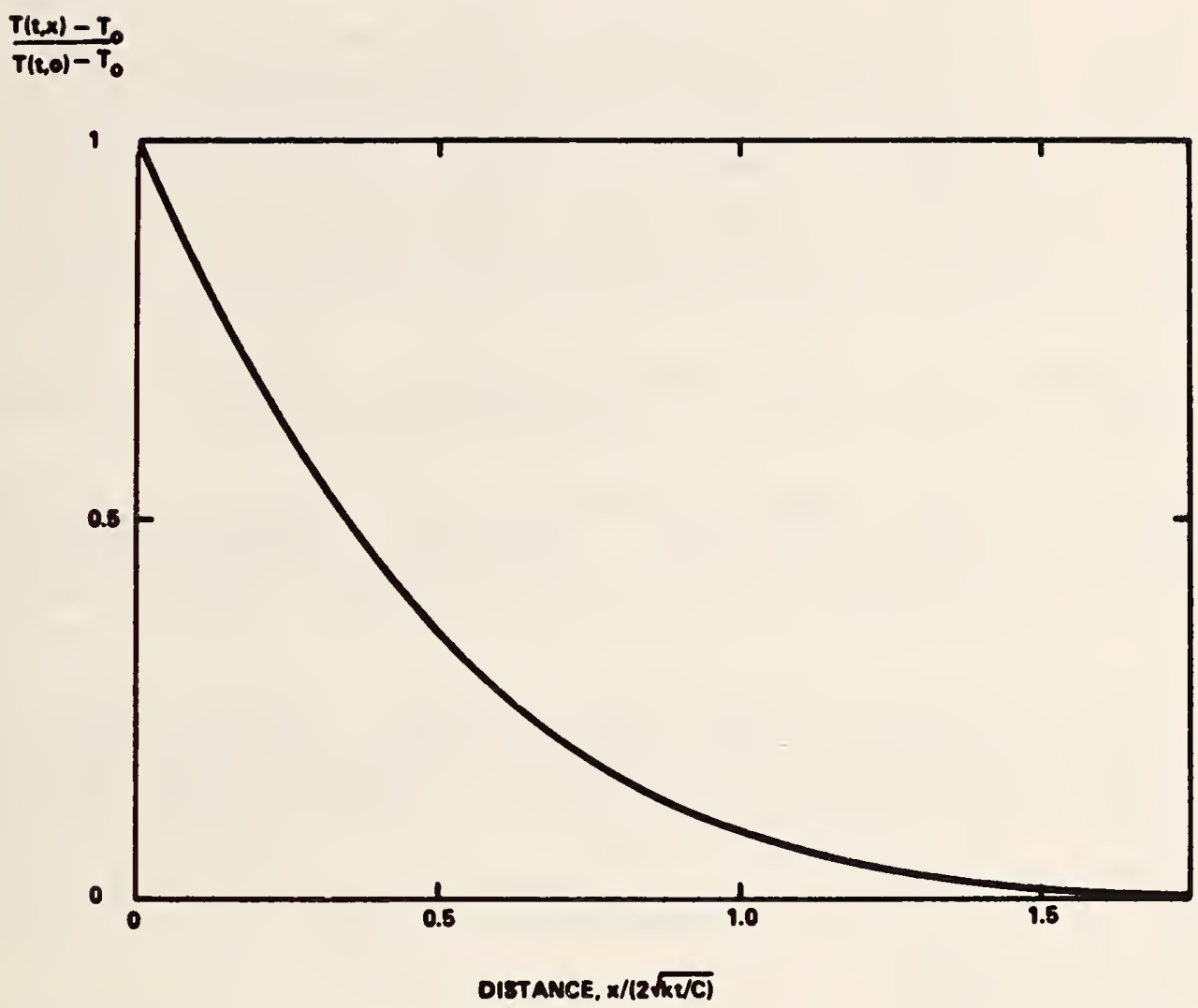

(a)

$\frac{\sqrt{x} A K}{x P}\left(T(L, x)-T_{0}\right)$

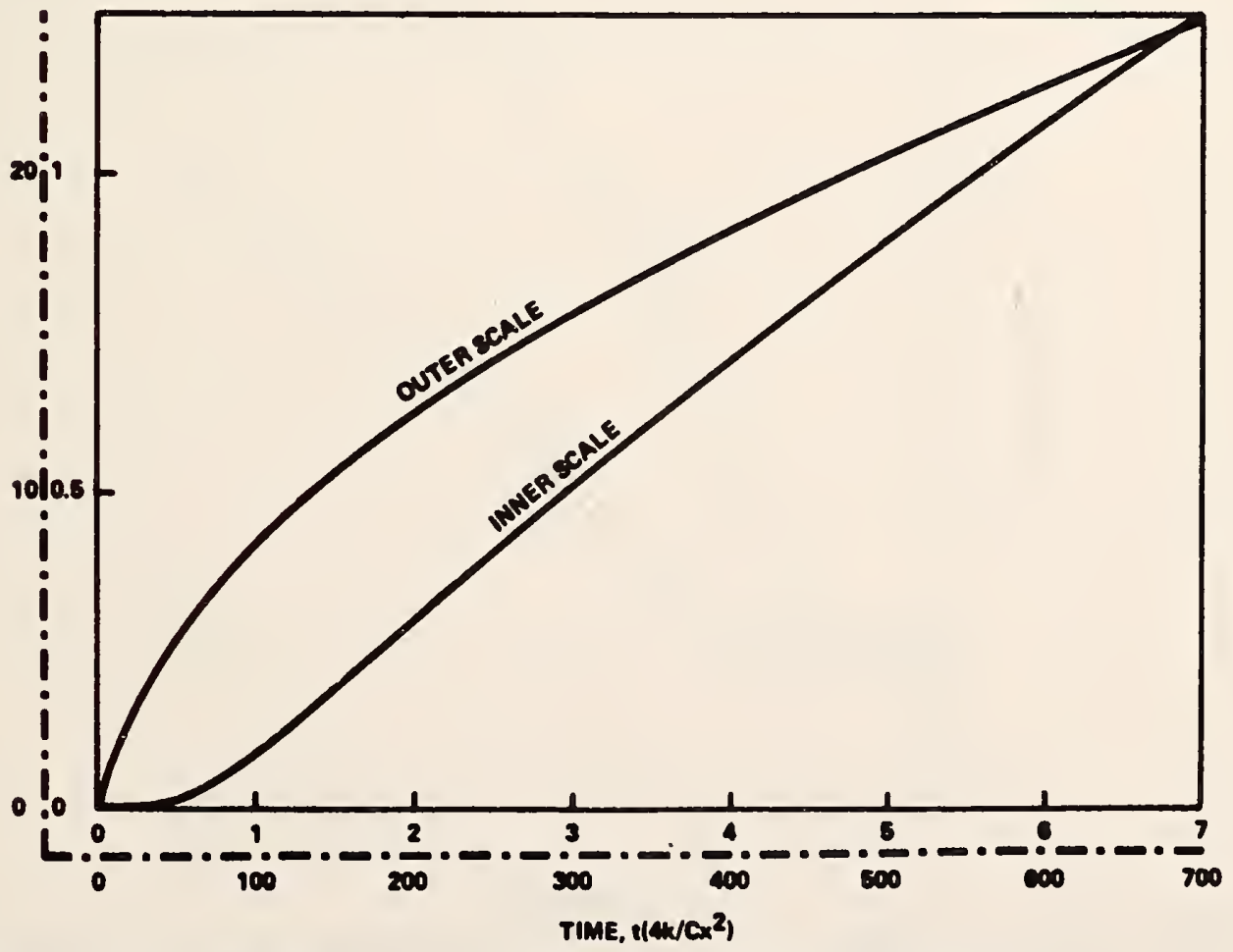

(b)

Fig. 2. Spatial (a) and temporal (b) solutions of diffusion equation. 
Our method of measurement is to record the temperatures, $T_{1}$ and $T_{2}$, as a function of time. This gives a pair of curves of the shape of Fig. $2 b$, but with different time scales since $T_{1}$ changes more rapidly after the power is turned on than $T_{2}$, and of course $T_{2}$ is always less than $T_{1}$. From these curves we can read off a pair of ${ }^{2}$ numbers $T_{1}$ and $T_{2}{ }^{2}$ for a particular time $t^{\prime}$. For that particular time we then have both coordinates ${ }^{1}\left(T_{1}, x_{1}^{2}\right)$ and $\left(\mathrm{T}_{2}, \mathrm{x}_{2}\right)$ for two points on the curve of Fig. 2a, which establishes the horizontal and vertical scales of that curve. This process involves use of the ratios $x_{1} / x_{2}$ and $T_{2} / T_{2}$ and an interactive procedure (using a computer) which locates the two unique poilits having the correct values of these ratios. The ratio, $\mathrm{k} / \mathrm{C}$, is obtained directly from the horizontal scale factor and the product $k C$ can be derived at $x=0$ from the vertical scale factor and equation (2). The combination of this ratio and product yield the values of $k$ and $C$.

It is only essential, of course, to measure $T_{1}$ and $T_{2}$ at some convenient time $t$. However, measuring $T_{2}$ and $T_{2}$ as a function of time provides a high degree of redundancy which is useful in checking for errors and inconsistencies in the method.

Since $k$ and $C$ are usually temperature dependent at low temperatures, it is important to choose a power level and time scale such that $T_{3}$ and $T_{2}$ do not rise very much above the initial value $T_{0}$. Otherwise, the temperature dependence of $k$ and $C$ must be considered in equation (2), making the analys is difficult if not impossible. The use of small temperature intervals is standard practice in specific heat and thermal conductivity measurements.

Since the bar is not semi-infinite, the measurement time $t$ ' should be chosen small enough that no appreciable amount of heat reaches the far end of the bar, otherwise the spatial and temporal profiles given in Fig. 2 will no longer be accurate. Since the spatial profile, Fig. 2a, approaches zero very quickly at large $x$, it seems satisfactory to choose $x_{2}$ (the position at which $T_{2}$ is measured) at about the middle of the real bar, and then choose $t^{\prime}$ such that the rise in $T_{2}$ is no more than about $25 \%$ of the rise in $T_{1}$. It is assumed that the position $x_{1}$ should be near the free end of the bar. Any deviation of the temporal profile from the curve of Fig. $2 b$ might indicate that one or more of these conditions has been violated.

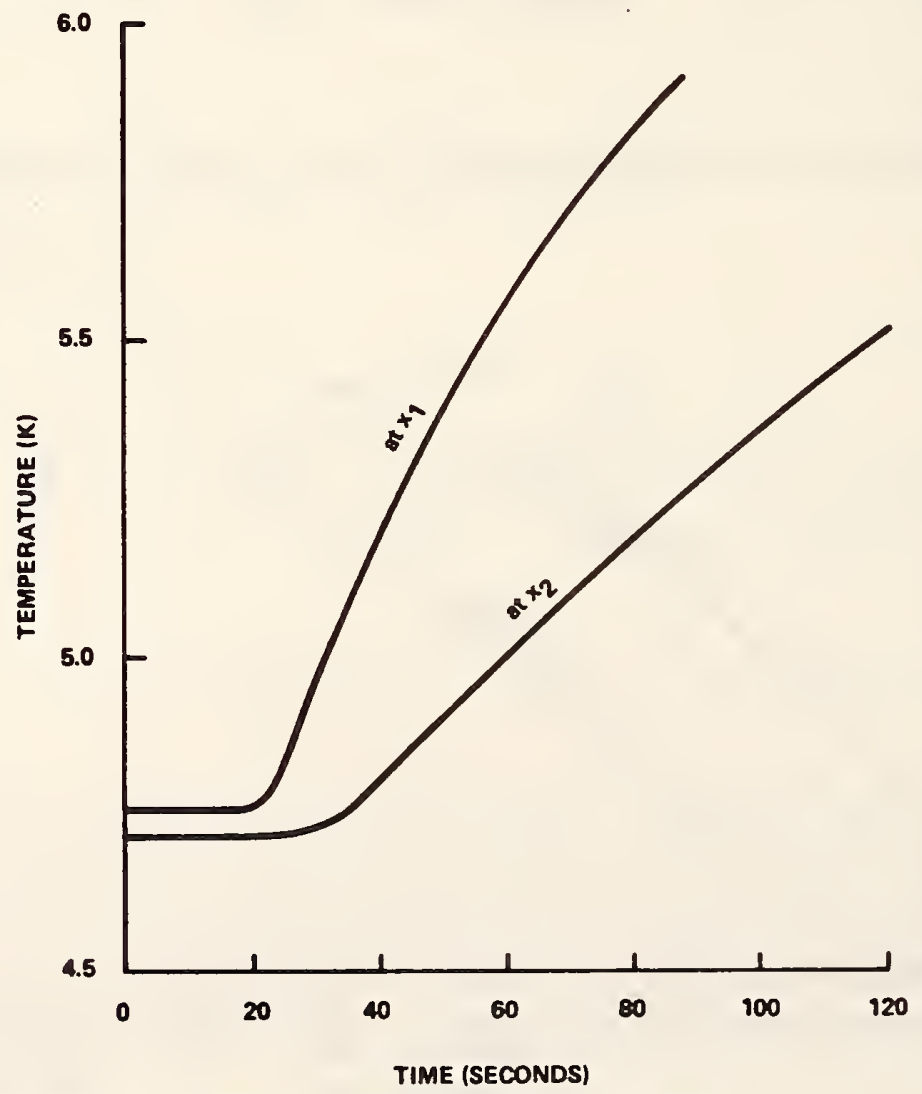

Fig. 3. Temperature as a function of time for two thermometers on a G-10 epoxy fiberglass rod. The thermometers were $1.2 \mathrm{~cm}$ and $4 \mathrm{~cm}$ from the heater. The rod had a crosssectional area of $0.895 \mathrm{~cm}^{2}$. 


\section{Results}

Figure 3 shows the temperature at 2 thermometers on a G-10 epoxy-fiberglass rod as a function of time after a step function was applied to the heater. The rod area was $.895 \mathrm{~cm}^{2}$ and the thermometers were located $1.2 \mathrm{~cm}$ and $4.0 \mathrm{~cm}$ from the heater.

We found that the consistency of the results was poor if we selected a time $t$ for which the temperature change at the second thermometer was small. This is presumably related to problems of resolution in the temperature at this point. We also found that the results varied considerably if the heat wave was allowed to reach the heat sink (the temperature controlled end of the bar) before the temperature points were taken. This clearly relates to a breakdown in the assumption that the rod was semi-infinite.

For the data of Fig. 3, reasonable consistency in results was achieved over the time interval of 40 to 75 seconds. While the temperature differences are a bit larger than desired, we used the method described in the previous section to determine the thermal conductivity and specific heat of this material at $5 \mathrm{~K}$. 3 The thermal conductivity was found to be $1.1 \mathrm{~mW} / \mathrm{cm}-\mathrm{K}$ and the specific heat was $4.7 \mathrm{~mJ} / \mathrm{cm}^{3}-\mathrm{K}$.

These numbers fall within the range of values given by other investigators. Additional work must be done to arrive at a quantitative criterion for selection of valid data reqions. The measurement scheme is to be automated so as to provide for rapid acquisition of information over a modest temperature range for a variety of materials. One should note that this technique has been devised to deal with rapid evaluation of a wide range of materials. An accuracy of $10-20 \%$ should suffice for such a study and this method seems well suited to the task.

\section{References}

[1]. G. C. Danielson and P. H. Sidles, Thermal Diffusivity and Other Non-Steady-State Methods, in Thermal Conductivity, edited by R. P. Type, 149-200 (Academic Press, New York, NY, 1969). 
U.S. DEPT. OF COMM.

BIBLIOGRAPHIC DATA

SHEET (See instruction s)
1. PUBLICATION OR REPORT NO.

IBS TN-1049
2. Performing Organ. Report No.

3. Publication Date

January 1982

4. TITLE AND SUBTITLE

\section{A Study of Design Principles for Refrigerators for Low-Power Cryoelectronic Devices}

5. $A \cup T H O R(S)$

J. E. Zimmerman and D. B. Sullivan

6. PERFORMING ORGANIZATION (If joint or other thon NBS, see instructions)

7. Contract/Grant No.

NATIONAL BUREAU OF STANDARDS

DEPARTMENT OF COMMERCE

8. Type of Report \& Period Covered

WASHINGTON, D.C. 20234

9. SPONSORING ORGANIZATION NAME AND COMPLETE ADDRESS (Street, City, Stote, ZIP)

10. SUPPLEMENTARY NOTES

Document describes a computer program; SF-185, FIPS Software Summary, is attached.

11. ABSTRACT (A 200-word or less foctual summory of most significont informotion. If document includes a significont bibliography or literature survey, mention it here)

This report summarizes a five-year effort at NBS which has been directed toward the development of low-power cryocoolers suited to the support of superconducting instruments. The report deals with a variety of aspects of construction and operation of refrigerators as well as with a model which allows one to optimize the design for minimum drive power. The publications generated by the program are included as an appendix.

12. KEY WORDS (Six to twelve entries: alphabeticol order: copitalize only proper names; ond seporote key words by semicolons) Cryocooler; cryogenics; low temperature; refrigerator; Stirling cycle; superconducting devices.

13. AVAILABILITY

X] Unlimited

For Official Distribution. Do Not Release to NTIS

Order From Superintendent of Documents, U.S. Government Printing Office, Washington, D.C. 20402.

[X] Order From National Technical Information Service (NTIS), Springfield, VA. 2216I
14. NO. OF PRINTED PAGES

114

15. Price

$\$ 11.00$ 


\section{NBS TECHNICAL PUBLICATIONS}

\section{PERIODICALS}

JOURNAL OF RESEARCH-The Journal of Research of the National Bureau of Standards reports NBS research and development in those disciplines of the physical and engineering sciences in which the Bureau is active. These include physics, chemistry, engineering, mathematics, and computer sciences. Papers cover a broad range of subjects, with major emphasis on measurement methodology and the basic technology underlying standardization. Also included from time to time are survey articles on topics closely related to the Bureau's technical and scientific programs. As a special service to subscribers each issue contains complete citations to all recent Bureau publications in both NBS and nonNBS media. Issued six times a year. Annual subscription: domestic $\$ 16$; foreign $\$ 20$. Single copy, $\$ 3.75$ domestic; $\$ 4.70$ foreign.

NOTE: The Journal was formerly published in two sections: Section A "Physics and Chemistry" and Section B "Mathematical Sciences."

DIMENSIONS/NBS-This monthly magazine is published to inform scientists, engineers, business and industry leaders, teachers, students, and consumers of the latest advances in science and technology, with primary emphasis on work at NBS. The magazine highlights and reviews such issues as energy research, fire protection, building technology, metric conversion, pollution abatement, health and safety, and consumer product performance. In addition, it reports the results of Bureau programs in measurement standards and techniques, properties of matter and materials, engineering standards and services, instrumentation, and automatic data processing. Annual subscription: domestic \$11; foreign $\$ 13.75$.

\section{NONPERIODICALS}

Monographs-Major contributions to the technical literature on various subjects related to the Bureau's scientific and technical activities.

Handbooks-Recommended codes of engineering and industrial practice (including safety codes) developed in cosperation with interested industries, professional organizations, and regulatory bodies.

Special Publications-Include proceedings of conferences sponsored by NBS, NBS annual reports, and other special publications appropriate to this grouping such as wall charts, pocket cards, and bibliographies.

Applied Mathematics Series-Mathematical tables, manuals, and studies of special interest to physicists, engineers, chemists, biologists, mathematicians, computer programmers, and others engaged in scientific and technical work.

National Standard Reference Data Series-Provides quantitative data on the physical and chemical properties of materials, compiled from the world's literature and critically evaluated. Developed under a worldwide program coordinated by NBS under the authority of the National Standard Data Act (Public Law 90-396).
NOTE: The principal publication outlet for the foregoing data is the Journal of Physical and Chemical Reference Data (JPCRD) published quarterly for NBS by the American Chemical Society (ACS) and the American Institute of Physics (AIP). Subscriptions, reprints, and supplements available from ACS, 1155 Sixteenth St., NW, Washington, DC 20056.

Building Science Series-Disseminates technical information developed at the Bureau on building materials, components, systems, and whole structures. The series presents research results, test methods, and performance criteria related to the structural and environmental functions and the durability and safety characteristics of building elements and systems.

Technical Notes-Studies or reports which are complete in themselves but restrictive in their treatment of a subject. Analogous to monographs but not so comprehensive in scope or definitive in treatment of the subject area. Often serve as a vehicle for final reports of work performed at NBS under the sponsorship of other government agencies.

Voluntary Product Standards-Developed under procedures published by the Department of Commerce in Part 10, Title 15, of the Code of Federal Regulations. The standards establish nationally recognized requirements for products, and provide all concerned interests with a basis for common understanding of the characteristics of the products. NBS administers this program as a supplement to the activities of the private sector standardizing organizations.

Consumer Information Series-Practical information, based on NBS research and experience, covering areas of interest to the consumer. Easily understandable language and illustrations provide useful background knowledge for shopping in today's technological marketplace.

Order the above NBS publications from: Superintendent of Documents, Government Printing Office, Washington, DC 20402.

Order the following NBS publications-FIPS and NBSIR's-from the National Technical Information Services, Springfield, VA 22161.

Federal Information Processing Standards Publications (FIPS PUB)-Publications in this series collectively constitute the Federal Information Processing Standards Register. The Register serves as the official source of information in the Federal Government regarding standards issued by NBS pursuant to the Federal Property and Administrative Services Act of 1949 as amended, Public Law 89-306 (79 Stat. 1127), and as implemented by Executive Order 11717 (38 FR 12315, dated May 11, 1973) and Part 6 of Title 15 CFR (Code of Federal Regulations).

NBS Interagency Reports (NBSIR) - A special series of interim or final reports on work performed by NBS for outside sponsors (both government and non-government). In general, initial distribution is handled by the sponsor; public distribution is by the National Technical Information Services, Springfield, VA 22161, in paper copy or microfiche form. 
U.S. DEPARTMENT OF COMMERCE

National Bureau of Standards

Washington, D.C. 20234

OFFICIAL BUSINESS

Penalty for Private Use. $\$ 300$
POSTAGE AND FEES PAID U.S. DEPARTMENT OF COMMERCE COM-215

SPECIAL FOURTH-CLASS RATE BOOK

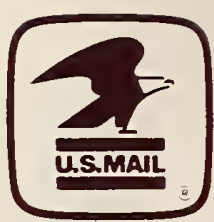

